\title{
Lock-Free Concurrent Binomial Heaps
}

\author{
Gavin Lowe \\ Department of Computer Science, University of Oxford; gavin.lowe@cs.ox.ac.uk
}

\begin{abstract}
We present a linearizable, lock-free concurrent binomial heap. In our experience, a binomial heap is considerably more complex than previously considered concurrent datatypes. The implementation presents a number of challenges. We need to deal with interference when a thread is traversing the heap, searching for the smallest key: our solution is to detect such interference, and restart the traversal. We must avoid interference between updating operations: we add labels to nodes to prevent interfering updates to those nodes; and we add labels to heaps to prevent union operations interfering with other operations on the same heap. This labelling blocks other operations: to achieve lock-freedom, those threads help with the blocking operation; this requires care to ensure correctness, and to avoid cycles of helping that would lead to deadlock. We use a number of techniques to ensure decent efficiency. The complexity of the implementation adds to the difficulty of the proofs of linearizability and lock-freedom: we present each proof in a modular way, proving results about each operation individually, and then combining them to give the desired results. We hope some of these techniques can be applied elsewhere.
\end{abstract}

Keywords: Concurrent datatypes, binomial heaps, linearizability, lock-freedom.

\section{Introduction}

A binomial heap is a datatype that stores a multiset of integer keys. It has operations as declared to the right (we use Scala notation). The effect of the operations is as follows.

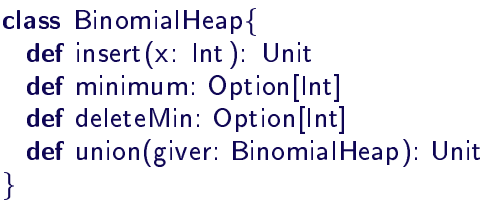

- The operation insert inserts the given value into the heap.

- The operation minimum returns the minimum key in the binomial heap; in order to deal with an empty heap, it returns an Option[Int] value: either a value Some $(x)$ where $x$ is the minimum key, or None if the heap is empty.

- The operation deleteMin deletes and returns the minimum key (again, using an Option[Int] value).

Preprint submitted to Journal of Logical and Algebraic Methods in ProgrammingAugust 21, 2018 
- The operation union removes all keys from the argument heap ("giver") and adds them to the current heap. We call the former heap the giving heap, and the latter the receiving heap.

In this paper we describe an implementation of a concurrent binomial heap. Our implementation is linearizable and lock-free (see below).

We start by reviewing sequential binomial heaps. Binomial heaps were introduced by Vuillemin in [1]; Cormen et al. [2] give a good description.

A binomial tree is composed of nodes. Each node $n$ holds an integer key, denoted $n$.key. A binomial tree of degree 0 comprises a single node. A binomial tree of degree $d>0$ has a root node, and $d$ subtrees with degrees $d-1, d-2$, $\ldots, 1$, respectively; the root's key is no larger than any other key in the tree. Given two trees $t_{1}$ and $t_{2}$, each of degree $d$, with $t_{1}$.key $\leq t_{2}$.key, we can merge them into a tree of degree $d+1$ by grafting $t_{2}$ onto $t_{1}$ as its first child. A binomial tree of degree $d$ has $2^{d}$ nodes.

A binomial heap is a list of zero or more binomial trees, with their roots linked together; we call this list the root list. Each node has a reference next to its next sibling or the next node in the root list, and a list children of references to its children; for convenience, in our concurrent implementation each node also has a reference parent to its parent. The figure to the right illustrates a binomial heap containing four trees, with degrees 1, 3, 1 and 0 . (Each next reference is drawn rightwards;

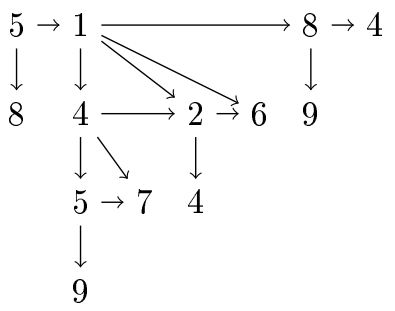
references to children are drawn downwards; parent references are omitted.)

The sequential implementation of a binomial heap ensures the trees in a heap have different degrees, by merging two trees of the same degree, if necessary. This means that a heap with $k$ keys has at most $\lfloor\log k\rfloor+1$ trees, thereby providing $O(\log k)$ running times for each of the operations. Our concurrent implementation does not rigidly enforce this property, but we aim towards it, in the interests of efficiency. (The sequential implementation also keeps the list in increasing order of degrees; we make no attempt to achieve this property.)

We now sketch how the operations are implemented in a concurrent setting. The minimum operation scans the root list for the node with the minimum key that was not deleted when encountered. Then, if that node has not been deleted in the meantime, it returns the key; otherwise, it restarts. The deleteMin operation again scans the root list for the node with the minimum key. It then attempts to mark that node, thereby indicating that it has claimed the node for deletion. If successful, it then removes that node from the root list, inserting its children (if any) in its place. If the deletion is unsuccessful, it restarts. The insert operation for a key $k$ traverses the root list until it finds either a root of degree 0 with a key that is no larger than $k$, or the last root node. In the former case, it tries to insert a new node below the singleton root; in the latter case, it tries to insert a new node after the last root. In each case, if it is unsuccessful 
it retries. The union operation similarly tries to append the first root of the giving heap after the last root of the receiving heap. We provide two implementations of union: the former makes the simplifying assumption that there are no concurrent operations on the giving heap (although concurrent operations are allowed on the receiving heap); the latter removes this assumption, at the cost of a much more complex implementation.

In addition, threads may attempt to merge two trees into a single tree, via a non-public operation merge; this shortens the root list, thereby making other operations more efficient. The merge operation takes two trees $a$ and $b$ that are expected $^{1}$ to have the same degree with a.key $\leq$ b.key, and a node pred that is expected to be the predecessor of $b$. It attempts to merge $a$ and $b$ into a single tree, making $\mathrm{b}$ a child of a (so a is above and $\mathrm{b}$ is below). For the moment, we assume that threads can call this operation at any time, nondeterministically. In Section 10 we explain our tactic for using it.

There are several issues that make the implementation difficult. First, suppose one thread is traversing the root list, searching for the minimum key. Meanwhile, other threads could rearrange the heap concurrently; this can cause the traversing thread to miss nodes in the heap. For example, suppose a traversing thread has reached the 3 in the left-most heap below, and then is suspended. Then suppose other threads merge the 3 below the 1 ; merge the 2 below the 1 ; and then delete the 1 , reaching the right-most heap below.

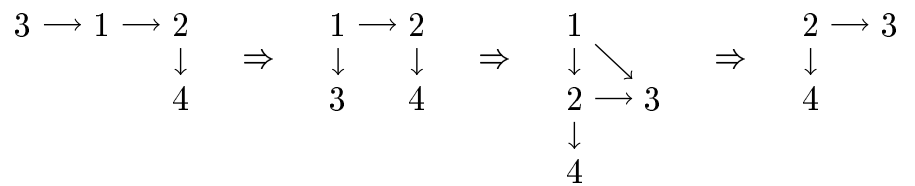

Now, if the traversing thread resumes, it detects that it has reached the end of the root list, and incorrectly returns 3. (This problem arises even if the other operations are performed atomically.) Our solution is to detect when the root list has been sufficiently perturbed, and restart the traversal.

Another major difficulty, of course, is that most updates to the heap involve updating more than one node in the heap. For example, merging trees a and $b$, making the latter a child of the former, involves updating both these nodes and the predecessor pred of $b$, so involves up to three nodes (it is possible that $\mathrm{a}=$ pred). Deleting a node involves updating the node itself (to mark it as deleted), its predecessor, and each of its children (to clear their parent references). Hence such updates cannot be made atomically and efficiently on standard architectures. This leads to intermediate states that are different from ones that can arise when the operations are atomic.

Our approach to merging is based on the technique of Barnes [3]. We start by labelling relevant nodes. Those labels are removed when the operation is com-

\footnotetext{
${ }^{1}$ We use the word "expected" to indicate conditions that the thread in question should check before the operation is called, but that subsequently might have been invalidated.
} 
pleted, or if the operation is unable to complete and has to be backtracked. The labels indicate the intended update to other threads. Informally, this locks those nodes, preventing other threads from interfering with the operation; however, other threads are allowed to update the node to help to complete or backtrack the operation; we use the word "locks" in this sense below. Similarly, when deleting a node, we start by giving it a Delete label; other threads may update this node and its children only to complete the operation or to backtrack it.

Extra difficulties arise if we allow operations concurrent to a giving union (i.e. where the heap is the giving heap in a union). The union may interfere with other operations, for example causing an insertion to actually insert the new node in the receiving heap, or for a minimum or deleteMin to return a key from the receiving heap. Our approach to avoiding such problems extends the above labelling technique. We add a label to the head node of each heap during a union; likewise, we add a label to the head node for the critical step of insertions and deletions: this ensures that these operations cannot happen concurrently to a giving union. We also need efficient techniques to detect if a giving union has happened during some period, and to find the current heap containing a node.

Finally, we aim to make the data structure lock-free: if threads (collectively) are scheduled sufficiently often, then eventually some operation completes. This means that if a thread is blocked by the labelling of nodes described in the previous paragraph, that thread should help to complete the operation. Ensuring that no thread can get stuck in a loop requires great care.

Our approach to proving lock-freedom is as follows. For most operations, each update to a node represents positive progress: we can bound the number of updates that an operation makes, excluding helping. Thus, in any execution that represents a failure of lock-freedom, such updates must eventually end. The exception to this is with merging; we use a different mechanism to bound the number of such updates done by merges. Thus, it is enough to prove that each operation terminates in a finite number of steps assuming no other thread updates a node. We do this by showing three things (under this assumption):

- That each operation terminates in a finite number of steps, other than, perhaps, helping another operation;

- Likewise that each attempt to help terminates in a finite number of steps, other than, perhaps, recursively helping another operation;

- That each chain of recursive helping is finite (in particular, acyclic).

In our experience, a binomial heap is considerably more complex than previous concurrent datatypes. Part of our goal in undertaking this work was to understand the degree to which concurrent versions of more complex datatypes are possible, and how best to reason about them. (Our proofs are rigorous but not machine-checked; we leave a machine-checked proof as a challenge.)

We consider our main contributions to be the combination of techniques that we used to overcome the above challenges and to improve the efficiency of the implementation, together with the verification. In addition, we consider the 
binomial heap potentially useful in its own right: in several use cases, our implementation out-performs other implementations of concurrent priority queues (i.e. omitting the union operation from the interface); further, we are not aware of other lock-free implementations giving the same interface (including union).

During the development, we intensively tested the implementation for linearizability [4] using the techniques from [5]. This revealed several subtle errors with earlier versions of the implementation. It is our experience that this technique is very effective at discovering errors, if they exist; conversely, not finding errors improves our confidence, and justifies the effort of a formal proof.

Our implementation, in Scala, is available from http://www.cs.ox.ac.uk/ people/gavin.lowe/BinomialHeap/index.html. We present the design below in pseudo-Scala (taking a few small liberties with syntax).

The implementation of each operation is, to a certain extent, dependent on the others; we describe the operations in a slightly unnatural order, in order to produce a coherent explanation. In the next section we define the types of nodes, and give some basic definitions. In Section 3 we describe how to merge two trees. In Section 4 we describe how to delete a node, assuming the minimum node and its expected predecessor have been found; we also assume a function to find the new predecessor of the node being deleted, if it has changed in the meantime; we present that latter function in Section 5, where we also present our technique for safely traversing the root list. In Section 6 we describe the insert operation. In Sections 7 we describe the union operation under the assumption that there are no concurrent operations on the giving heap. In Sections 8 and 9 we describe the minimum and deleteMin functions. We describe our tactic for when we attempt to merge trees in Section 10. In Section 11 we present a revised version of union, where we relax the assumption about no concurrent operations on the giving heap; this also requires making some small changes to the previous operations, which we describe in Section 12. We prove that the implementation is linearizable in Section 13, and we prove lock-freedom in Section 14. We sum up in Section 15. In places, in the interests of clarity, we present slightly simplified versions of the implementation, and then sketch enhancements without giving full details. We omit some proofs, in the interest of brevity: these can be found in [6].

Linearizability. Our correctness condition is the well-established and accepted condition of linearizability [4]. Informally, a concurrent datatype $C$ is linearizable with respect to a sequential (specification) datatype $S$ if every history $c$ of $C$ is linearizable to a history $s$ of $S$ : each operation $o p$ of $c$ appears to take place atomically at some point in time, known as the linearization point, between the invocation and return of $o p$; and the operations, ordered according to their linearization points, form a legal history of the sequential datatype $S$ (i.e. respects the intended sequential semantics). See $[4,7]$ for a formal definition; but this definition suffices for our purposes.

Related work. To our knowledge, the only prior implementation of a concurrent binomial heap is by Crupi et al. [8]. However, their setting is very different from 
ours: a single operation takes place at a time, and all threads cooperate on each operation. They show each of the main operations can be performed on a heap containing $n$ keys in time $O\left(\log \log n+\frac{\log n}{p}\right)$ with $p=O\left(\frac{\log n}{\log \log n}\right)$ processors.

Huang and Weihl [9] describe a lock-based priority queue based on Fibonacci heaps, where deleteMin operations are non-strict, i.e. they may return values that are not necessarily minimal.

In addition, various authors have given implementations of priority queues (without the union operation) based on skiplists. Lotan and Shavit [10] describe a design that is not linearizable, since a deleteMin operation may ignore a smaller value that is inserted concurrently. Further, the design uses locks, so is clearly not lock-free. Sundell and Tsigas [11] describe a lock-free priority queue based on a skiplist. The design also turns out not to be linearizable ${ }^{2}$; but this is easily fixed. Lindén and Jonsson [12] describe a linearizable skiplist-based implementation of a priority queue, that uses various clever techniques to minimise the number of update operations necessary for a deleteMin operation, and so provide good performance.

Liu and Spear [13] describe an implementation of a priority queue using a mound: a binary heap where each node holds a linked list sorted by priorities; insertion runs in $O(\log (\log (N)))$ and deleteMin in $O(\log (N))$.

Braginsky et al. [14] present an implementation of a priority queue based upon a linked list of chunks, where a chunk uses an array to store some items of data. Each chunk holds data that are smaller than those in the following chunk; but only the data in the first chunk (on which deleteMin operates) are kept sorted. When a chunk becomes full, it is split into two. A skip list allows fast access to the appropriate chunk. A form of elimination between concurrent insert and deleteMin calls is implemented.

Some papers, e.g. [15], have considered relaxed priority queues, where the deleteMin operation is not constrained to return the absolute minimum key; this avoids the minimum key becoming a bottleneck, so gives better performance.

\section{Basic types and definitions}

In this section we describe the implementation of nodes. Each node has the following fields:

- key: Int, which is immutable;

- parent, next: Node (each possibly null), pointing to the node's parent, and next sibling or next root, respectively;

- children: List[Node], a list of the node's children (possibly empty);

- degree: Int, giving the degree of the node;

\footnotetext{
${ }^{2}$ Acknowledged by the authors (personal communication).
} 


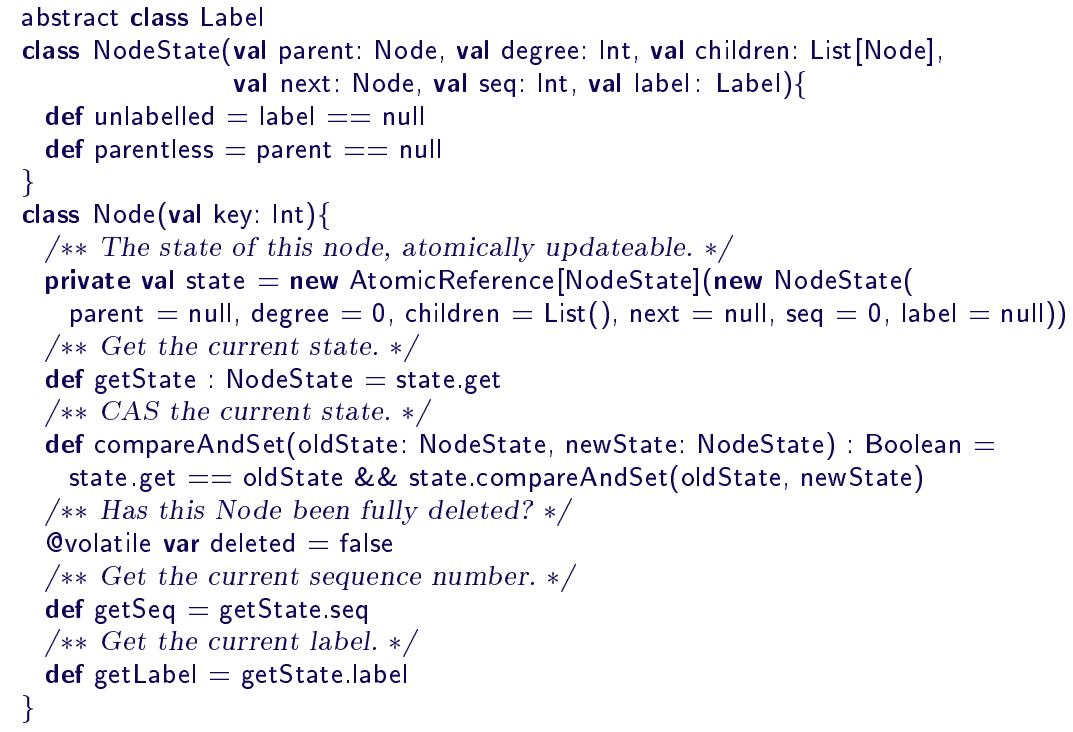

Figure 1: NodeState and Node

- label: Label (possibly null), describing any update currently operating on the node; we introduce the different types of labels when we describe the relevant operations;

- seq: Int, a sequence counter that is updated each time a node is moved from being a root to become a child of another node, or when the node is labelled for deletion; we use this when traversing the root list, to detect changes in the root list that require the traversal to restart (see Section 5);

- deleted: Boolean, a flag that is set when a node has been fully deleted; we explain this more when we discuss deletion in Section 4.

To update several parts of a node's state atomically, we encapsulate most of the mutable state into a NodeState object (with immutable fields); see Figure 1. We use suggestive notation for defining new NodeState objects: we write, for example, state[next $\mapsto \mathrm{n}$ ] for a NodeState that is the same as state except with its next field set to point to $\mathrm{n}$. We include in NodeState functions that test if the label or parent is null.

A Node itself comprises the (immutable) key, a reference state to the state, and the deleted field ${ }^{3}$ (we do not include deleted within the NodeState, because

\footnotetext{
${ }^{3}$ The class java.util.concurrent.atomic. AtomicReference provides references to objects which can be updated atomically, using compare-and-set operations, and that provide sequentially consistent memory accesses. The @ volatile annotation provides sequentially consistent memory accesses to that field.
} 
we never need to update it at the same time as another field, nor in a way that depends on any other field). Each Node includes operations to (atomically) read the state, perform a compare-and-set $(\mathrm{CAS})^{4}$ on the state, and to get the sequence number or label. In our informal commentary, we sometimes write, e.g., $n$.next as shorthand for $n$.getState.next.

The binary heap is implemented as a linked list of root nodes, linked via their next fields; we call this list the root list. For convenience, we use a dummy header node head (with an arbitrary key).

private val head $=$ new $\operatorname{Node}(-1)$

For simplicity, we assume a garbage collector. Whenever we update the state of a node, we do so with a new object; this avoids the ABA problem [7].

We state some invariants of the binomial heap. In [6], we show that each operation maintains each of these invariants; these are mostly straightforward checks. The following invariants capture the standard property of keys in a binomial tree, and some other obvious properties.

Invariant 1. If $n_{2}$.parent $=n_{1}$ and $n_{1} \neq$ null then $n_{1} . k e y \leq n_{2} . k e y$.

Recall that we use a Delete label to indicate that a node is being deleted.

Invariant 2. For each node $n$ that does not have a Delete label: n.children contains n.degree nodes, which are joined by next pointers in a linked list; and c.parent $=n$ for each $c \in$ n.children.

Invariant 3. The parent references are acyclic.

Labels are added only to root nodes, except a Delete label may be added to a node whose parent is being deleted.

Invariant 4. If a node has a non-null parent, then either it has a null label, or both it and its parent have Delete labels.

(We state further invariants later.)

We give rules under which operations may change nodes.

Rule 1. If a node $n$ has a non-null parent, the only changes that may be performed on $n$ are to help with the deletion of the parent, or to label $n$ for deletion if its parent is being deleted; in each case the parent will have a Delete label.

We make this more precise in Section 4 when we describe deletion. Our other rules concern nodes with labels, restricting the updates that may be performed on the node, often to just updates that help to complete the corresponding operation, or to remove the label if the operation is backtracked. We give these rules when we present the corresponding labels. When we analyse an operation, we do so under the assumption that all other operations follow these rules. We verify that this is indeed the case when we analyse those other operations.

\footnotetext{
${ }^{4}$ compareAndSet(oldState, newState) behaves as follows: if state equals oldState, then it is updated to newState, and true is returned; otherwise, state is unchanged, and false is returned. We check that the state equals oldState before attempting the CAS operation: this is standard practice, to avoid creating lots of memory bus traffic via a CAS that is bound to fail.
} 


\section{Merging trees}

The merge function merges two trees a and $\mathrm{b}$, so that a ends up above, and b below. More precisely, it takes three trees, a, b and pred (where possibly $\mathrm{a}=$ pred, but otherwise the trees are distinct), and their expected states aState, bState and predState. It assumes that a.key $\leq$ b.key, predState.next $=$ b, aState and bState have the same degrees, and all are unlabelled roots with null parents. It attempts to merge $a$ and $b$, making a the parent of $b$. Recall that for the moment we assume that threads can call merge at any time, nondeterministically, subject to the above precondition; in Section 10, we explain our tactic for using it.

The merge function is a private function: it is done only to optimise performance by reducing the length of the root list. We therefore don't care much if a call to merge is unsuccessful: if another operation interferes with it, it will detect that the state of a node has changed (which might invalidate the conditions for the merge), backtrack by undoing its previous steps, and give up; however, we do ensure that it terminates in a finite number of steps.

We start by sketching how the operation proceeds, and then flesh out the details, below. Suppose, for the moment, a $\neq$ pred. The merge function proceeds as follows; the steps are illustrated in Figure 2.

1. It tries to add a MergeParent label to a. We call this the a-labelling step. If the state of a has changed, this fails, and merge gives up.

2. It tries to add a MergeNext label to pred. We call this the pred-labelling step. If this fails (and no other helping thread has added the MergeNext label), it backtracks, removes the label from a, and gives up.

3. It tries to update $b$, atomically setting its parent field to a, and its next field to a's first child, or to null if a has no children. We call this the b-update step. If this fails (and no other thread has done the update), it backtracks, removing the labels from pred and a, and gives up. If this update succeeds, the merge is guaranteed to complete successfully.

4. It tries to update pred, atomically setting its next field to bState.next, and removing the label. We call this the pred-update step.

5. It tries to update a, atomically incrementing its degree, adding $b$ to its children, and removing the label. We call this the a-update step.

All steps except for the a-labelling may be performed by other helping threads. Note that if one of the labelling steps fails, because the state of the relevant node has changed, then the merge gives up: in most such cases, it is not possible to complete the merge, because the relevant parts of the precondition no longer hold. Note that it is fine to just give up, since merging is just an optimisation.

If $\mathrm{a}=$ pred, things are slightly simpler. The pred-labelling can be omitted, and subsumed into the a-labelling. Similarly, the pred-update and a-update can be combined.

The two labels contain enough information to allow helping threads to complete the operation; they are defined as follows.

case class MergeParent(pred: Node, predState: NodeState, pLabel: MergeNext,

b: Node, bState: NodeState) extends Label

case class MergeNext(a: Node, b: Node, bState: NodeState) extends Label 


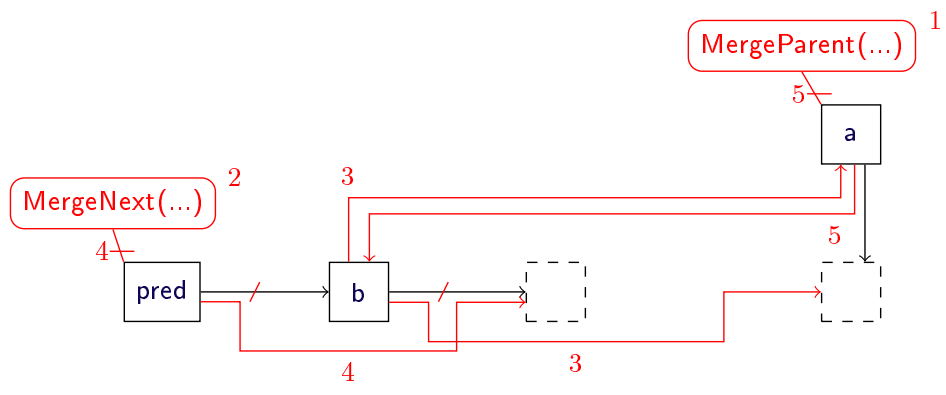

Figure 2: Illustration of the merge function. next references are drawn to the right, parent references upwards, and references to the first child downwards; dashed boxes illustrate possibly null nodes; updates are indicated in red.

Within MergeParent, if a = pred the pLabel parameter is bound to the corresponding MergeNext label for pred; if a = pred, it is bound to null; each other parameter is bound to the corresponding parameter of merge.

In more detail, the function merge (Figure 3 ) attempts the a-labelling. It prepares the two labels and, if the states have not changed, attempts to label a. This locks a and its children. The fact that this thread prepares both labels means that the MergeNext label is uniquely identified with this invocation of merge, and cannot be confused with another label with identical arguments. ${ }^{5}$

The function mergeLabelPred attempts the pred-labelling. If successful, this locks pred and its children. If the labelling is unsuccessful, and no other thread has performed this labelling, it backtracks and removes the label from a.

The b-update step is attempted by the function mergeUpdateB. It attempts to update b, setting its parent and next fields appropriately. If this is successful, the merge is guaranteed to complete. If the update is unsuccessful, and no other thread has performed this step, then it attempts to backtrack.

When pred $\neq a$, the pred update and a updates are performed by the functions mergeUpdatePred and mergeUpdateA (Figure 4), respectively ${ }^{6}$. The case pred $=a$ is handled by mergeUpdateAPred.

The following rule concerns MergeParent and MergeNext labels.

Rule 2. If a node has a MergeParent label, the only updates allowed on it are to perform the a update, to remove the label if the merge cannot be completed, or to perform the pred update if $a=$ pred. If a node has a MergeNext label, the only updates allowed on it are to perform the pred update, or to remove the label if the merge cannot be completed.

\footnotetext{
${ }^{5}$ We use "eq" and "ne" for equality and inequality tests between non-null labels; these correspond to reference equality: using "==" and "!=" would correspond to value equality (this is the default for case classes), which would be incorrect in some places.

6 "::." represent cons, adding an element to the front of a list.
} 


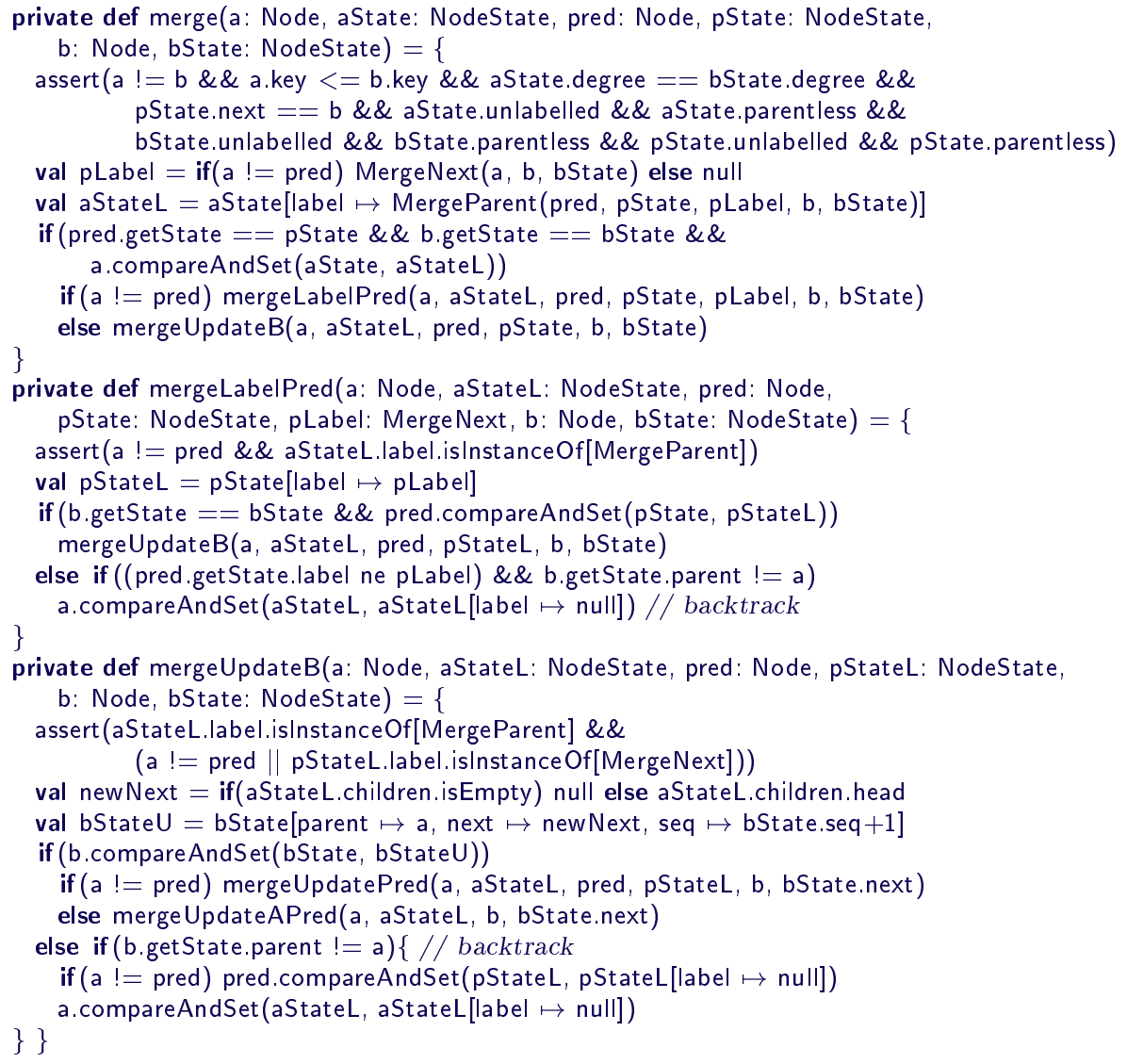

Figure 3: The merge, mergeLabelPred and mergeUpdateB functions.

If a thread performing another operation is unable to proceed because it is blocked by the merge, then it helps the merge; this might be because the other operation needs to update a node that has been labelled by the merge, but is unable to do so because of the above rule. Thus once the initial labelling step has happened, some of the steps may be performed by such helping threads. Any thread helping does so via the help function in Figure 5, which calls into the appropriate sub-function of merge ${ }^{7}$ (line 25 concerns helping with deletions; we explain this case in Section 4). We design the code within the merging functions to allow such helping: updates on nodes take place only from the expected states.

\footnotetext{
${ }^{7}$ The code makes use of Scala pattern matching. The pattern in line 16, using "@", binds the variable pLabel to the label. The pattern in line 19, using back ticks, matches just a MergeParent label whose fields match the given values.
} 


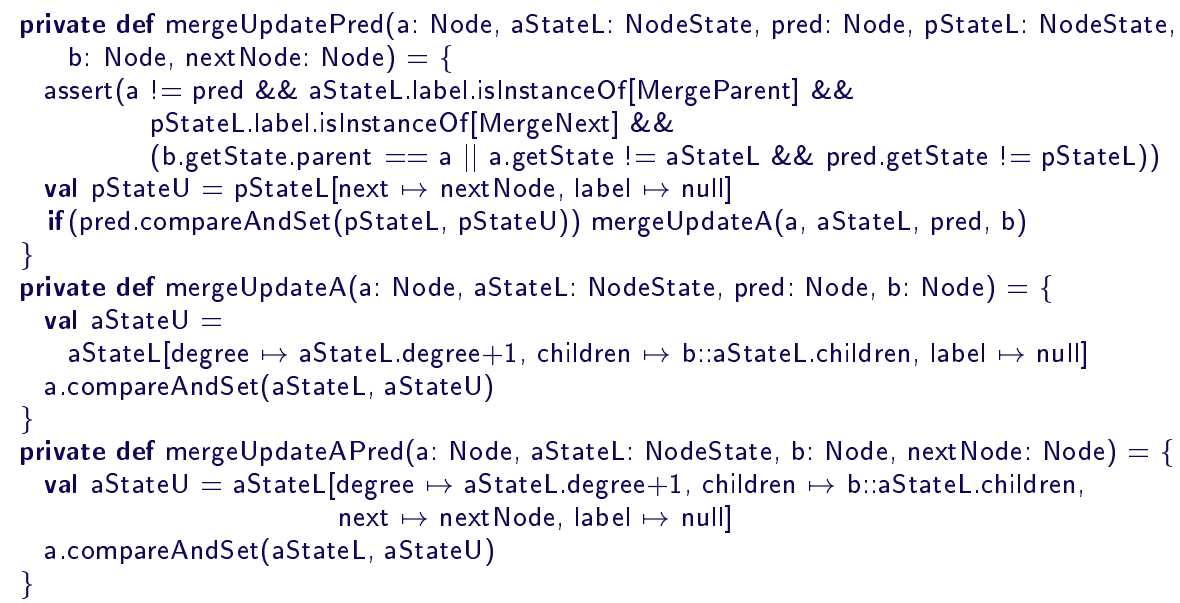

Figure 4: The mergeUpdatePred, mergeUpdateA and mergeUpdateAPred functions.

At various places, a thread may detect that another thread has already performed the next step. For example, in mergeLabelPred, the thread may detect that another thread has already labelled pred (line 19 of Figure 3). In such cases, it allows the thread that performed that step to complete the operation. This is for pragmatic reasons, since it is inefficient for two live threads to be working on the same operation: one of the threads could be doing something else; and if both threads were working on the same operation, then when one thread performs a particular CAS operation, it would invalidate the relevant cache lines of the other thread, forcing extra memory accesses.

We now argue the correctness of the merge operation.

Proposition 1. A call to merge can be either successful or unsuccessful: a successful call merges the two trees; an unsuccessful call leaves the heap essentially unchanged, maybe just replacing node states with equivalent ones with the same fields.

Proof: (Sketch.) First, note that the implementation works correctly when it operates without interference from other threads (assuming the stated precondition): each step of the operation succeeds, and the final states of the three nodes correspond to the merge having completed.

Now suppose that a thread helps via help. Then help calls into the correct sub-function, such that the prerequisite steps have already been performed. Note that the order in which b's and pred's states are read in lines 3 and 4 of Figure 5 is critical: in order to be sure that the pred update has happened at line 8 , we need to be sure that pred's state was not read before the pred-labelling step. Helping threads contribute correctly to the deletion. Each update on a node is executed only from an appropriate state: either the state that was passed to the initial call of merge and subsequently stored in a label, or the state 


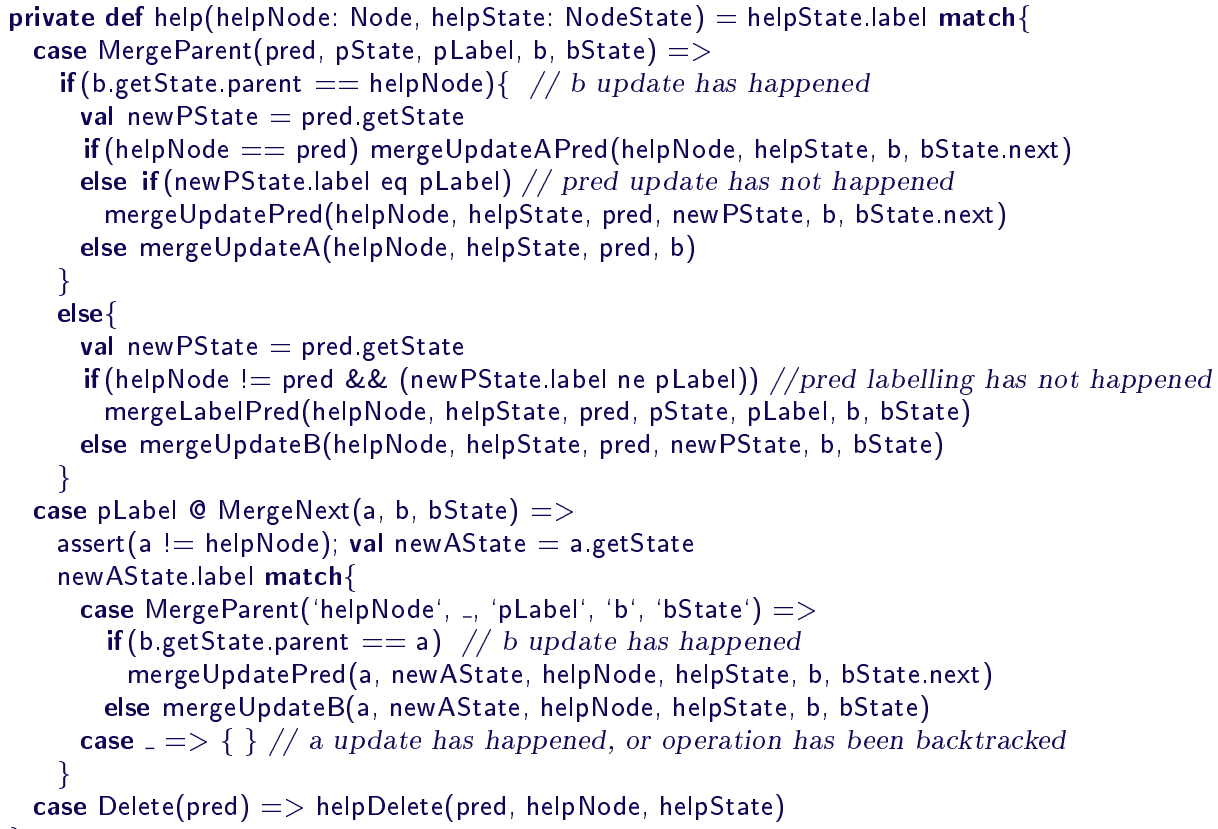

Figure 5: Helping operations.

resulting from the relevant labelling; hence if several threads attempt such an update, only the first succeeds.

Note in particular that backtracking is done appropriately: if a thread starts to backtrack, another will not perform updates to try to complete the merge. Recall that after the b update, the merge is guaranteed to complete, so cannot be backtracked. If it is necessary to backtrack the pred labelling, we do so before backtracking the a labelling. In particular, the backtracking within mergeLabelPred (Figure 3) is done correctly: it won't be done if another thread has labelled pred, since in that case either: (a) pred still has the label pLabel (before the pred update), or (b) b's parent equals a (after the b update until after the a update), or (c) a's state has changed (after the a update); in each case, the backtrack CAS won't be attempted. Also note that the backtracking in mergeUpdateB (Figure 3) is done correctly: it won't be done if another thread has updated $b$ as part of the merge, since in that case either (a) b's parent equals a (until after the a update), or (b) the state of pred or a has changed (at the pred update or a update); in each case, the backtrack CAS won't be attempted.

We now show that other operations do not interfere with the correctness of merging. If any thread updates a before the a-labelling step, then the merge fails, and leaves the heap unchanged. Once a has been labelled, by Rule 2, no other operation may update it other than to help with the merging or backtrack 
it, until it is unlabelled in the a update. If any thread updates pred before the pred-labelling step, then the merge fails, the labelling of a is backtracked, and the heap is left essentially unchanged. Once pred has been labelled, by Rule 2, no other operation may update it other than to help with the merging or backtrack it, until it is unlabelled in the pred update; subsequent updates do not interfere with the merge. If any thread updates $b$ before the $b$ update, then the merge fails, the labelling of pred and $b$ are backtracked, and the heap is left essentially unchanged. After the $b$ update, but before the a update, no thread may update b: by Rule 1 , no thread may update it other than as part of the deletion of its parent; but its parent a still has a MergeParent label, so any attempt to delete a is blocked until the merge is complete.

We now state a lemma that will be useful for proving liveness properties.

Lemma 2. Any call to merge terminates in a finite number of steps. Each thread calls help on a node with a particular MergeNext or MergeParent label at most four times.

Essentially, for each call to help, some progress is made, either by this thread, or by some other thread after helpState was read: this creates a bound on the number of calls necessary. merge and its subfunctions contain no loop or recursion, so each call terminates in a finite number of steps. Full details are in [6].

\section{Deleting nodes}

In this section we describe how a node is deleted. In Section 9, we will describe how this is invoked by the deleteMin function. We assume here that the node delNode to be deleted and its expected predecessor pred have been identified. Recall that we allow only root nodes to be deleted; however, we allow a thread to claim a non-root node $n$ for deletion if its parent $p$ is being deleted; however, the deletion of $p$ must be completed before continuing with the deletion of $n$.

The deletion itself is encapsulated in functions delete and deleteWithParent, the latter dealing with the case of a non-root. The attempt to delete delNode may fail, for example if another thread has already claimed it for deletion; each function returns a boolean to indicate if it was successful. If it fails, the deleteMin function either calls the function again, if this could feasibly succeed, or identifies a new node to try to delete.

It is possible that the predecessor of delNode changes before the relevant step of the deletion. In this case, it is necessary to identify the new predecessor. We describe the search for the new predecessor in Section 5. Informal experiments suggest the predecessor is often unchanged, so recording it is worthwhile.

Deletion proceeds in several stages. We give a brief overview here, and give more detail below; the steps are illustrated in Figure 6 .

1. It attempts to label delNode with a Delete label. We call this the delNodelabelling step. If this is successful, the deletion is guaranteed to succeed.

2. If the parent of delNode is being deleted, it helps with this deletion. 


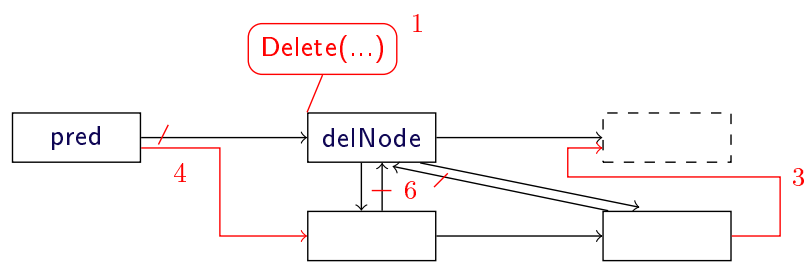

Figure 6: Illustration of deletion. Drawing conventions are as in Figure 2.

3. If delNode has children, the last child is updated to point to the successor of delNode.

4. If pred is still the predecessor of delNode, its next field is updated to point to delNode's first child, if it has one, or otherwise to delNode.next, thereby removing delNode from the root list; note that in the case that deINode has children, delNode.next is now reachable via the last child. If pred is not still the predecessor of deINode, a search is carried out to find the new predecessor, and the above update done to it. We call this the predupdating step.

5. delNode's deleted field is set, to indicate that it has been fully deleted.

6. The parent fields of delNode's children (if any) are cleared.

All steps except step 1 might be carried out by other threads helping.

Part of the rationale for our design is to allow a thread to claim the node it will delete, via the delNode-labelling step, as quickly as possible, so that threads waste as little time as possible in deletion attempts that subsequently fail. In a previous design, threads first labelled the precedessor pred, before labelling delNode (backtracking if the latter failed): this approach meant that the predecessor could not subsequently change, but proved slightly slower in practice. Similarly, we previously did not allow a non-root $n$ to be labelled for deletion; in such cases, all threads performing a deleteMin could identify $n$ as the minimum node, and all would help with the deletion of its parent before attempting to label $n$, which is inefficient; using our current approach, only the thread that succeeds in labelling $n$ helps with its parent.

We say that a node is marked for deletion (or just marked) if it has a Delete label. We say that it is decoupled if the pred update has occurred. We say that it is fully deleted if its deleted field is set.

We now make precise the circumstances under which a thread may change the state of a node with a non-null parent.

Rule 1. (refined) If a node $n$ has a non-null parent $p$, the only changes that may be performed on $n$ are as follows:

- if $p$ is marked for deletion, and $n$ is $p$ 's last child, then $n$ may have its next field set to p's successor;

- if $p$ is deleted, then $n$ may have its parent field cleared; 
- to mark $n$ for deletion.

The Delete label includes a reference to the expected predecessor node. It also includes a timestamp, giving the time that it was created: we use this in the minimum function to determine if the node was unmarked when the function was invoked.

case class Delete(pred: Node) extends Label \{ \}

val ts = java.lang.System.nanoTime // time label was created

Rule 3. If a node has a Delete label, the only subsequent changes allowed to it are to clear its parent field if its parent is deleted, or to set its deleted field once the node is decoupled. In particular, delNodenext does not change.

Deletion of a node with a null parent is encapsulated into the delete function (Figure 7). This takes as arguments the node delNode to be deleted, its expected state delState, and its expected predecessor pred. It calls labelForDelete, which tries to update delNode using a CAS, to add a Delete label and increase the sequence number; if successful, it returns the resulting state; in this case, the deletion is bound to succeed.

The function deleteWithParent deals with the case that the parent is non-null. Recall that in this case, all delNode's ancestors, up to the root list, are being deleted. It again calls labelForDelete to mark delNode for deletion; in the case that delNode.next is null, and hence delNode is parent's last child, it also updates delNode.next to parent.next, thereby performing the last child update on parent. If the marking is successful, it helps with the deletion of parent before completing the deletion of delNode.

The deletion is completed via the completeDelete function. If delNode has no children, it uncouples delNode from the previous node by updating the previous node's next field to point to delNode's successor, next, via the function predUpdate (see below). Otherwise, it first tries to update the last child's next pointer to point to the root node after delNode; this update fails only if it has already been performed by another thread. It then uncouples delNode via predUpdate, updating the previous node's next field to point to delNode's first child. Finally, it clears the parent field of each child.

The function predUpdate controls the update of the predecessor of deINode to point to newNext; the actual update and the setting of delNode's deleted field are done by the function completePredUpdate (Figure 8). delNode's deleted field is checked at various places, in case another thread has already completed the deletion. If pred is still the root node before delNode, completePredUpdate is called (line 4) to attempt to decouple delNode. Otherwise, or if the decoupling is unsuccessful, it is necessary to search for the new predecessor. The search is encapsulated in the function findPred. We present findPred in Section 5, and show that it satisfies the following property (proved as Lemma 12). The claim includes a premiss concerning the heap in which delNode might be; recall that we currently assume that the heap of the deletion is not concurrently the giving 


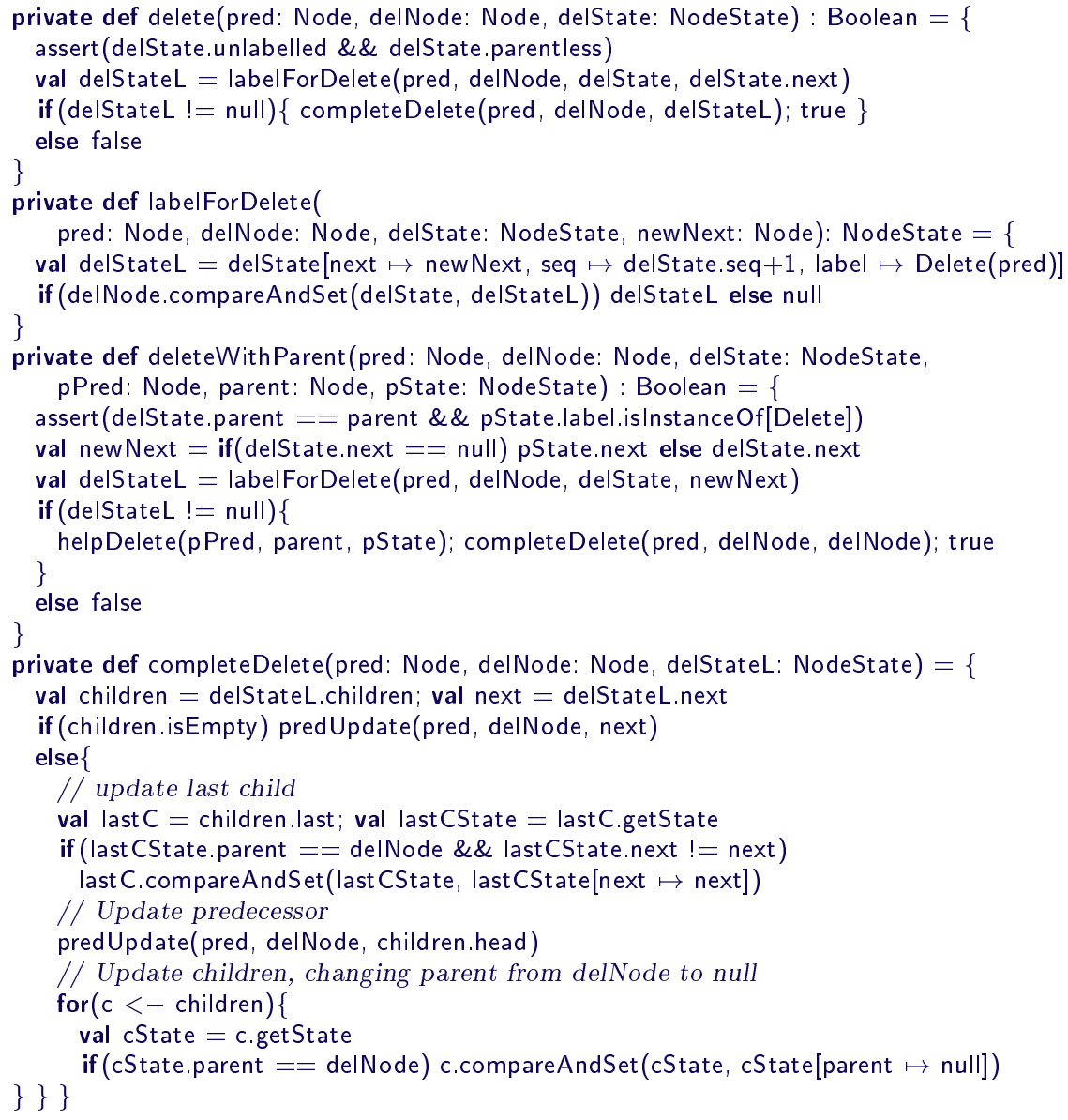

Figure 7: The delete, labelForDelete, deleteWithParent and completeDelete functions.

heap in a union, so deINode can be in no heap other than the heap of the deletion; we relax this assumption in Section 11, where this stronger form is useful.

Claim 3. Suppose delNode is marked for deletion and delNode.parentless. Consider a call to findPred(delNode) such that there is no giving union on the heap between the marking and findPred returning. Then findPred returns either:

1. a pair ( $p, p S t a t e)$, where $p$ State was a state of $p$ at some point during the call to findPred, such that $p$ State.next $=$ delNode and $p$ State.parentless; in this case, $p$ was not decoupled at the start of the call to findPred; or

2. the pair (delNode, null); in this case, the deletion of delNode has been completed.

In case 1, a call to completePredUpdate (line 11 of Figure 8) attempts to decouple 


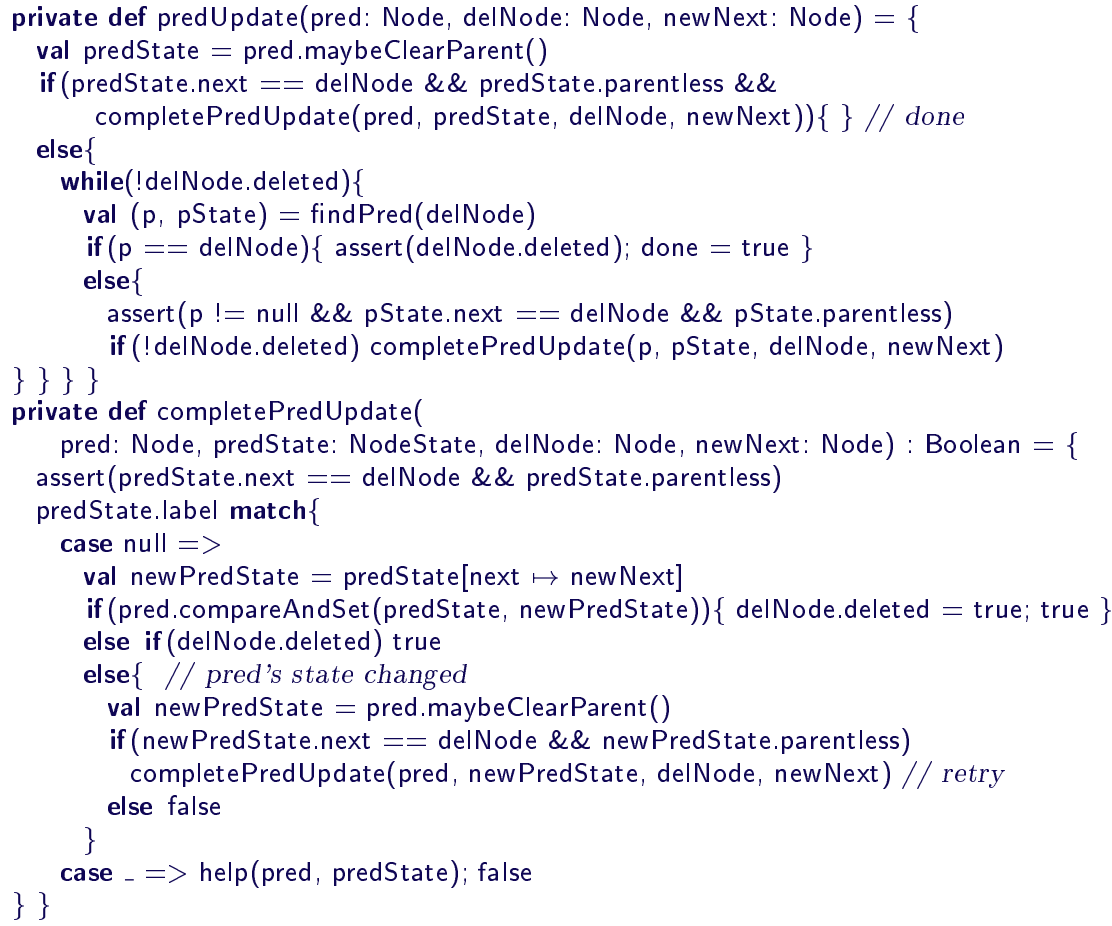

Figure 8: The predUpdate and completePredUpdate functions.

delNode from its expected predecessor p. In case 2 (line 8 ), the deletion has been completed by another thread.

The function completePredUpdate attempts to update pred's state from predState so that its next reference points to newNext. If this fails, and the deletion hasn't been completed by another thread, but pred's new state is such that the update is still possible, it recursively retries. It returns a boolean indicating whether the deletion has been completed.

Both predUpdate and completePredUpdate obtain pred's state via the function maybeClearParent (Figure 9), which has the side effect of clearing the parent field if the parent node has been deleted.

Any helping thread calls the helpDelete function in Figure 9 (perhaps via the help function from Figure 5).

We now sketch an enhancement to predUpdate. The search encapsulated by findPred is quite expensive, because it has to be resilient against other threads changing the root list. We can replace it by a less expensive but unreliable search (using a traversal similar in style to the one in Section 6), that may sometimes fail to find delNode even though it has not been decoupled from the root list. In this case, we need to repeat the search for pred. However, the unreliable version could fail to find pred repeatedly, which could represent a failure of lock- 


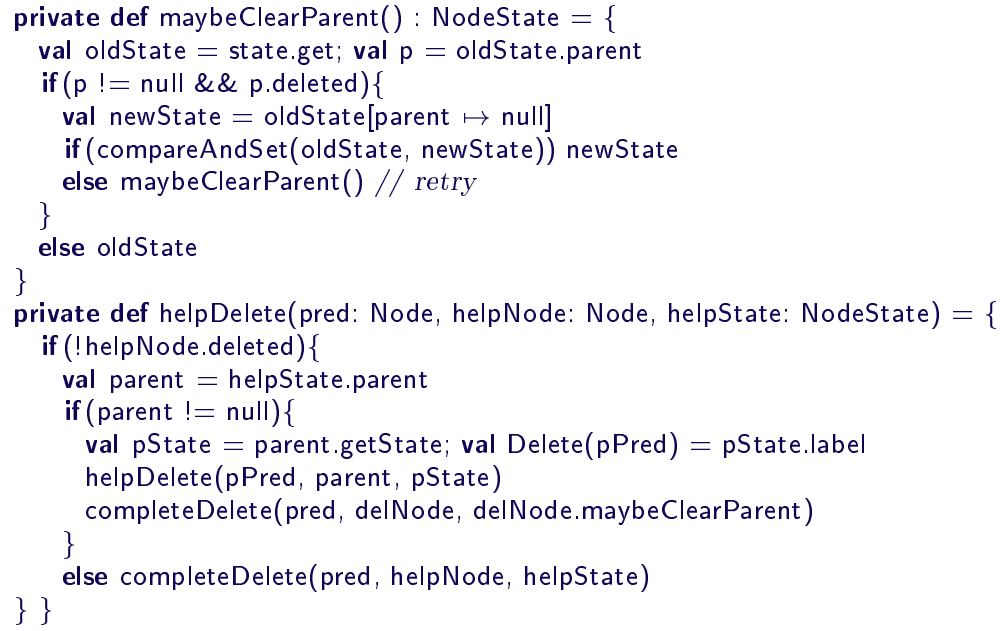

Figure 9: The maybeClearParent function (within the Node class), and the helpDelete function.

freedom. To avoid this, if the unreliable search fails and a subsequent search is required, we then use the findPred search. Our informal experiments suggest that the unreliable search is about three times faster than findPred, and that the subsequent search is very rarely necessary, making this enhancement worthwhile.

An additional enhancement is that if another thread traversing the root list finds a node that is partially deleted, but not uncoupled, it can help with that uncoupling.

We now state the correctness of the deletion. We assume here that Claim 3 holds; we discharge this assumption in Section 5.

Proposition 4. Assuming the stated preconditions, the delete function either deletes the node and returns true, or leaves the heap unchanged and returns false. The deleteWithParent satisfies a similar property, except may additionally help with the deletion of the parent.

The proof has a very similar style to the proof of Proposition 1, so we omit it; it can be found in [6].

We now give a partial liveness result for deletion. This lemma assumes limited interference from other threads. In Section 14, we build on this to prove lock-freedom. The lemma considers only steps performed outside findPred and the helping of another operation. In Section 5 we show that, under similar assumptions, calls to findPred take a finite number of steps. We then close the loop, and show that calls to delete and completeDelete take a finite number of steps in total.

Lemma 5. Consider an invocation of one of the functions of Figure 7, during which other threads make a finite number of updates to nodes. Then the call performs a finite number of steps except, possibly: (1) in calls to findPred within 
predUpdate, (line 7 of Figure 8); (2) in calls to helpDelete within deleteWithParent (line 18 of Figure 7); or (3) in calls to help within completePredUpdate (line 27 of Figure 8). In other words, it performs a finite number of steps in code presented in Figures 7 and 8, and in maybeClearParent.

\section{Finding the predecessor}

We now describe how the root list can be traversed in order to find the predecessor of a node that is being deleted. We use a very similar traversal method in Sections 8 and 9 to find the node with the smallest key. Recall, from the Introduction, that straightforward approaches risk missing relevant nodes if the root list is rearranged concurrently.

As we traverse the root list, when we first encounter a new root curr, we record its sequence number currSeq. If we subsequently find that curr's sequence number has changed -indicating that in the mean time curr has been merged below another node, or had a Delete label added - then we restart the traversal. Conversely, if the sequence number has not changed, the node has been a root throughout the intervening period.

In addition, while traversing, we skip over any node $d n$ with a Delete label: otherwise, once the deletion was complete, other threads could move $d n$.next around the heap; but the traversing thread would not notice this, since the state of $d n$ itself would not change.

Below, we present a subsidiary function advance that advances one step along the root list, and prove relevant properties. We then present the findPred function, used to find the predecessor of the node being deleted. We prove a correctness property, in particular that if the traversal does not find the node being deleted, then another node has decoupled it. We also give a liveness property.

We need to clarify what we mean by a root node. This is clear when there are no partially completed updates, but less clear otherwise. Informally, we consider a merge to take effect at the $b$ update: after this update, we define $b$ to no longer be a root. Similarly, we consider a delete to take effect when the node being deleted is decoupled: after this update, we define the node being deleted to no longer be a root. The following definition captures this formally.

Definition 6. We define a function $R:$ Node $\rightarrow$ List(Node) as follows ${ }^{8}$, such that $R(n)$ gives the list of root nodes starting from $n$.

- $R(n u l l)=\operatorname{List}()$.

- If $n$ has a MergeNext(a, b, bState) label or a MergeParent( $\left.n_{-,},-, b, b S t a t e\right)$ label (so, in each case, n.next = b), and b.parent $=a$ (so the b update has occurred), then $R(n)=n:: R$ (bState.next).

- Otherwise, $R(n)=n:: R(n . n e x t)$.

\footnotetext{
${ }^{8}$ We use Scala notation for lists: $\operatorname{List}(a, b, \ldots, z)$ represents a list containing $a, b, \ldots, z$; “::" represents cons; "+十" represents list concatenation.
} 
The root list of a heap is defined to be $R$ (head). We say that $r$ is a root if it is a member of the root list.

Note that the root list might contain partially deleted nodes, which have been given a Delete label but not yet decoupled.

Invariant 5. The root list is finite: in particular, it is acyclic.

The following lemma will be useful below. Recall that we currently do not allow giving unions (i.e. unions where this is the giving heap) concurrent to other operations on the same heap; we relax this in Section 11.

Lemma 7. Suppose node $n$, at some point in time, was a root node of heap $h$, had sequence number seq, and did not have a Delete label. If subsequently it still has sequence number seq, and there has been no intervening giving union on $h$, then it is still a root node of $h$, and still does not have a Delete label.

We use a subsidiary function advance (Figure 10) to help advance along the root list. It takes the current node curr and its expected sequence number currSeq; curr is assumed not to be marked for deletion unless its sequence number has changed (line 10). It normally returns a triple (next, nextSeq, skipNodes), where next is a subsequent root node (or null if the end of the root list has been reached), nextSeq is next's sequence number (or, arbitrarily, -1 if next is null), and skipNodes is a list of root nodes between curr and next that have either been marked for deletion or that are in the process of being merged below another node. We will show that, assuming curr was a root node, next was (at some point during the call of advance) also a root node, not marked for deletion, and had sequence number nextSeq, and that skipNodes held the intermediate root nodes. By Lemma 7, next remains a root node for as long as its sequence number is nextSeq. advance returns null (line 5) if the current node's sequence number has changed, to indicate a failure; in this case, the traversal restarts.

The subsidiary function skipDeleted(n) advances along the root list, skipping over nodes that have been marked for deletion. It returns a triple (next, nextSeq, dels) where next is the first root not marked for deletion (or null if there is no such), nextSeq is its sequence number (or -1 if next is null), and dels is the list of nodes marked for deletion between $n$ and next.

In most cases, advance returns the result of calling skipDeleted on curr.next. However, if curr's state changes after it is initially read (at line 4), then the call to advance restarts: this is necessary to be sure that the next node is still a root node at the point that its sequence number is read. Note, though, that the traversal does not need to restart in this case (unless the sequence number is subsequently found to have also changed).

The cases where the current node has a MergeNext label, or a MergeParent label corresponding to the case curr $=\mathrm{a}=$ pred, are handled by the function skipMerge. If the $b$ update has happened (line 27) then we skip over the next node (b), following the definition of the root list. Otherwise, if the state of $b$ is unchanged (line 26), then, for pragmatic reasons, we again skip over b: we 


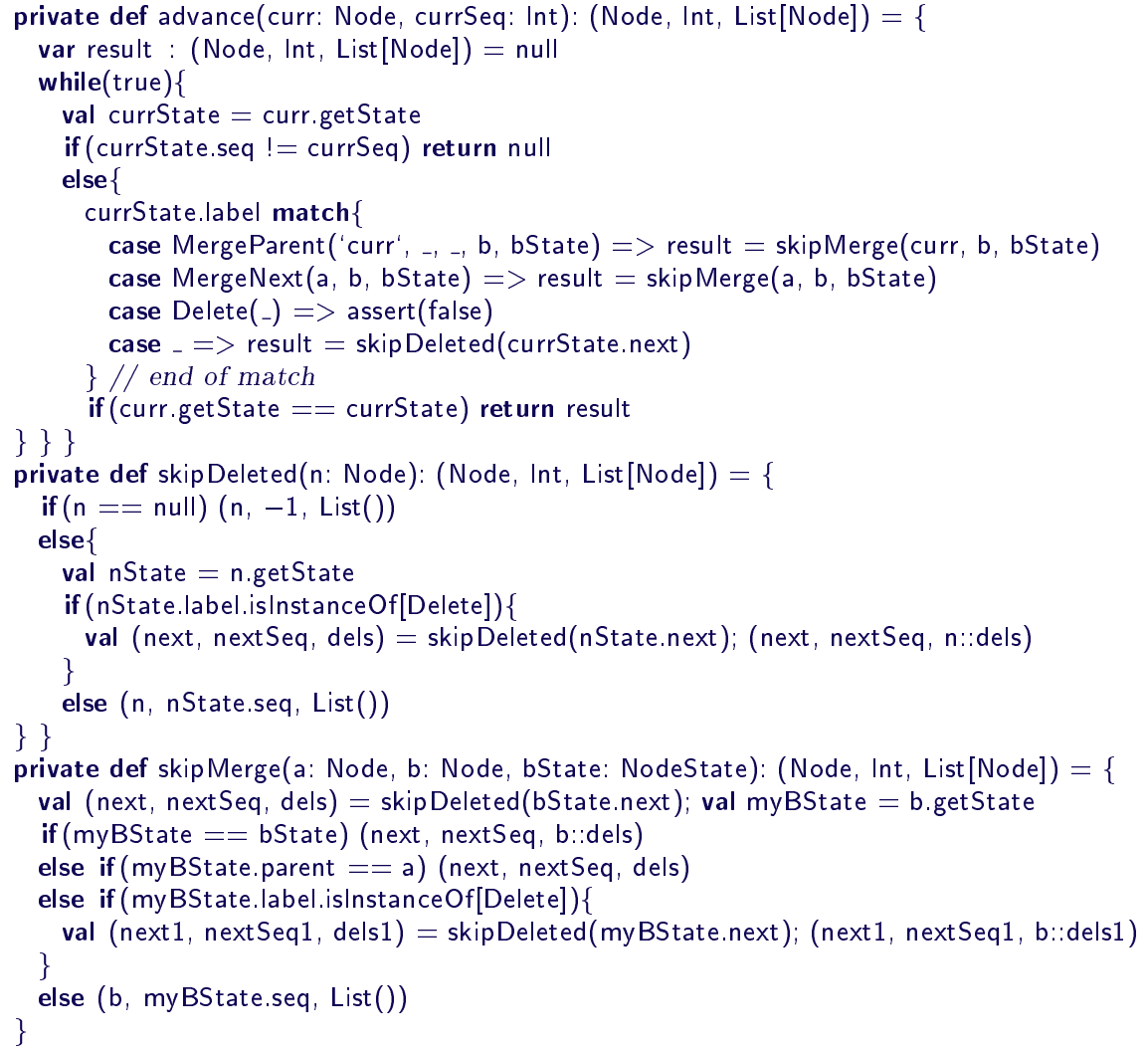

Figure 10: The advance, skipDeleted and skipMerge functions.

avoid alighting on $b$ since it will probably be updated soon, which could disrupt the traversal; however, we return $\mathrm{b}$ within the third component of the result. Finally, if the state of b has changed (lines $28-31$ ), we just advance in the normal way.

Lemma 8. Suppose skipDeleted is called on a node $n \neq$ null which is a root node of the current heap throughout the call, and suppose the call returns a triple (next, nextSeq, dels). Then at some time $t$ during the call:

1. If next $\neq$ null then next was a root node, had sequence number nextSeq, and was not marked for deletion.

2. The nodes returned were consecutive roots: $R(n)=$ dels $++R(n e x t)$. Further, this remains true for as long as n remains a root.

Proof: (Sketch; see [6] for details.) Recall (Rule 3), that once a node is marked for deletion, its next reference doesn't subsequently change. Hence if $n$ is the first of a sequence of deleted nodes, then those nodes remain roots for as long as $\mathrm{n}$ does. 
The following lemma captures the main properties of advance. It follows from Lemma 8 by careful checking of cases; the full proof is in [6].

Lemma 9. Suppose advance is called on a node curr that at some point in the past was a root node of heap $h$, had sequence number currSeq, and did not have a Delete label; and suppose there is no giving union on $h$ concurrent with the call. Suppose advance returns a triple (next, nextSeq, skipNodes). Then at some time $t$ during the call:

1. If next $\neq$ null then next was a root node of $h$, had sequence number nextSeq, and was not marked for deletion.

2. The nodes returned were consecutive roots:

$$
R(\text { curr })=\operatorname{List}(\text { curr })+\text { skipNodes }+R(\text { next }) .
$$

We now describe the function findPred (Figure 11), used by delete to find the predecessor of the node delNode being deleted if the latter is still in the heap. The traversal keeps track of the current node curr and its sequence number currseq. It uses advance to advance along the root list. If advance returns null, indicating that curr's sequence number has changed, the traversal restarts. Otherwise, a search is made through skipNodes for delNode and the node pred that was before it during the call to advance (line 8). If it finds delNode, and pred is still the previous root, pred and its state are returned (line 19).

If pred is not the previous node, it is possible that pred had a MergeNext or MergeParent node, and the $\mathrm{b}$ update had happened, so advance skipped over the $\mathrm{b}$ node. If the pred update has not yet happened, so pred still points to $\mathrm{b}$, it is necessary to help with the merge, to ensure lock-freedom (line 13). If pred is now the predecessor of delNode, the function returns; otherwise the traversal restarts.

Another complication arises if pred has a non-null parent parent. The most likely cause was that parent was decoupled from the root list before the traversal reached pred, but its deleted field has not yet been set. We help complete the deletion of parent, in order to ensure lock-freedom. If pred is now the predecessor of delNode, the function returns (line 24); otherwise it restarts the traversal.

If skipNodes does not contain delNode, the traversal advances (line 28). If the traversal reaches the end of the root list without finding delNode, it must have been detached by another thread: this thread sets the node's deleted field, and returns (delNode, null) to indicate that it has been detatched (line 30).

If $r$ is a root node, we write rootsUpTo(r) for the subsequence of the root list up to and including $r$, but excluding the dummy header.

Definition 10. If the root list is of the form List(head) $+x s++\operatorname{List}(r)+y s$, then rootsUpTo $(r)=x s++\operatorname{List}(r)$. We define rootsUpTo(head $)=\operatorname{List}()$. For convenience, we define roots $U p T o$ (null) to be all the root nodes excluding head.

The main invariant of the loop in findPred is that delNode does not appear in roots $U p T o$ (curr). The following lemma gives sufficient conditions for this property to be preserved by steps of other threads: any step that changes curr's 


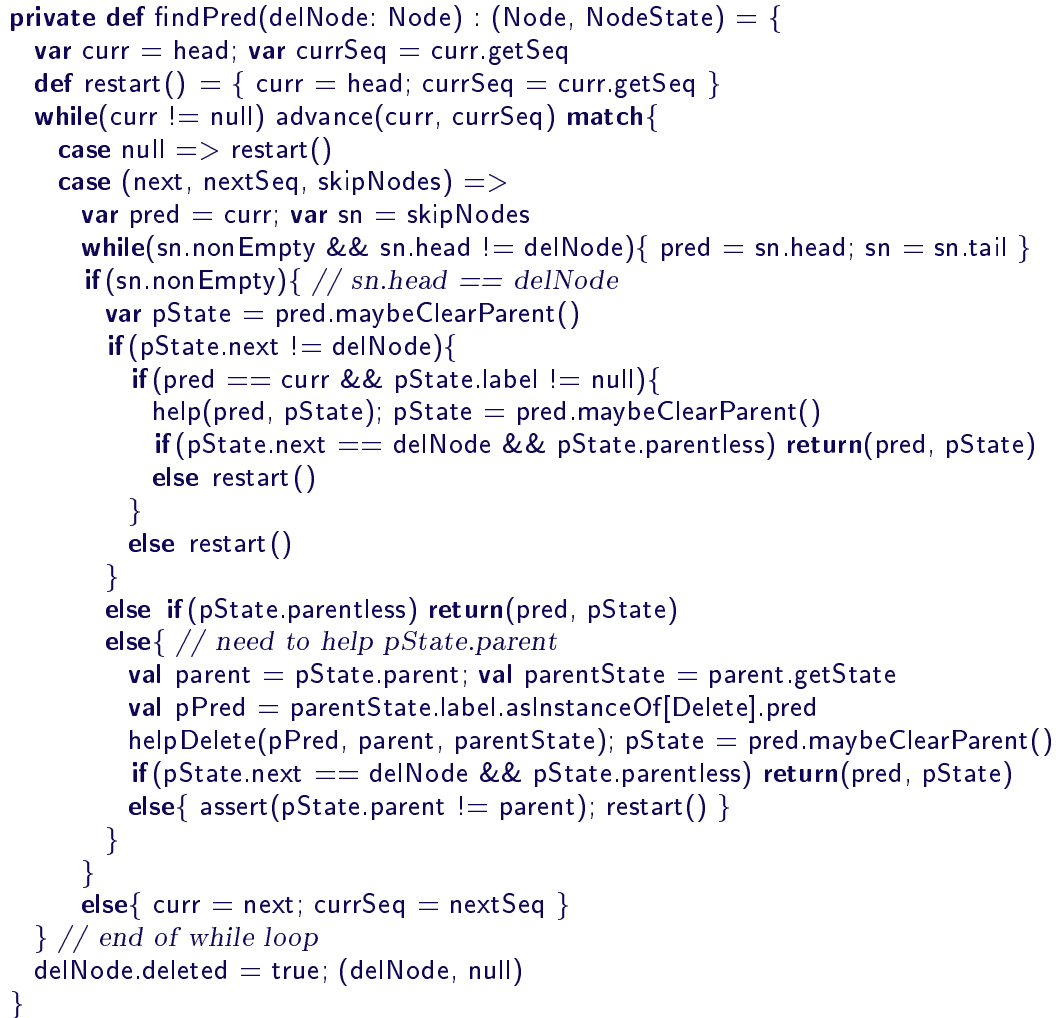

Figure 11: The findPred function.

position in the heap (by merging it below another node) also changes its sequence number; and no step moves delNode earlier in the heap (by merging it below an earlier root).

Lemma 11. Suppose node $d$ is marked for deletion, $d$.parent $=$ null, and $d \notin$ roots $U p T o(r)$. Consider a step of an operation that does not change the sequence number of $r$. Then subsequently still $d \notin$ roots $U p T o(r)$.

We can now verify findPred (presented earlier as Claim 3).

Lemma 12. Suppose delNode is marked for deletion and delNode.parentless. Consider a call to findPred(delNode) such that there is no giving union on the heap between the marking and findPred returning. Then findPred returns either:

1. a pair (pred, pState), where pState was a state of $p$ at some point during the call to findPred, such that $p$ State.next $=$ delNode and $p$ State.parent $=$ null; in this case, pred was not decoupled at the start of the call to findPred; or

2. the pair (delNode, null); in this case, the deletion of delNode has been completed. 
Proof: (Sketch; see [6] for details.) Note by the premiss about giving unions, delNode is in the heap unless it has been decoupled.

The following is invariant: if curr $\neq$ null and curr.getSeq $=$ currSeq, then curr is a root node and has not been marked for deletion. This can be shown using Lemmas 7 and 9. It's then easy to show that the returns at lines 19 and 24 satisfy case 1 .

Further, the following is invariant: if curr $=$ null or curr.getSeq $=$ currSeq, then delNode $\notin$ roots $U p T o$ (curr); this can be shown using Lemmas 9 and 11. Hence if the while loop terminates with curr $=$ null, delNode is not a root node of this heap. By assumption, it is not in any other heap. Hence it has been decoupled. The update to delNode.deleted completes the deletion, and the return at line 30 satisfies the second disjunct.

We now present a liveness result. We again assume limited interference from other threads.

Lemma 13. Consider an invocation of findPred, during which other threads make a finite number of updates to nodes. Then the invocation performs a finite number of steps, except, perhaps, within the call to helpDelete (line 23).

The following result follows directly from Lemmas 5 and 13 . We build on it to prove lock-freedom in Section 14; in particular, we show that any chain of helping is finite.

Proposition 14. Consider an invocation of either delete, deleteWithParent or completeDelete, during which other threads make a finite number of updates to nodes. Then the invocation returns after a finite number of steps other, perhaps, than steps helping other operations.

We now sketch a small enhancement to findPred. It is clear that the traversal can reach a child of delNode, or delNode.next, only after delNode has been decoupled. Hence we can use either delNode's first child or delNode.next as a sentinel in the guard of the while loop: if the sentinel is found, we can again complete the deletion (at line 30 ).

\section{Insertion}

Inserting a new key $k$ can be done by adding a new node containing $k$ either (a) after the final root node, or (b) as the child of a root of degree 0 whose key is no larger than $k$. The former approach is the more obvious. We include the latter approach for two reasons. Firstly, it avoids the last root node becoming a bottleneck. Secondly, it shortens the root list, and in particular means that if another thread is traversing the root list, it isn't racing against more and more root nodes being added.

The insert function is in Figure 12. It traverses the root list, keeping track of the current node in curr. It is possible that this traversal is disrupted by other threads rearranging the heap, but this does not matter in this case. 


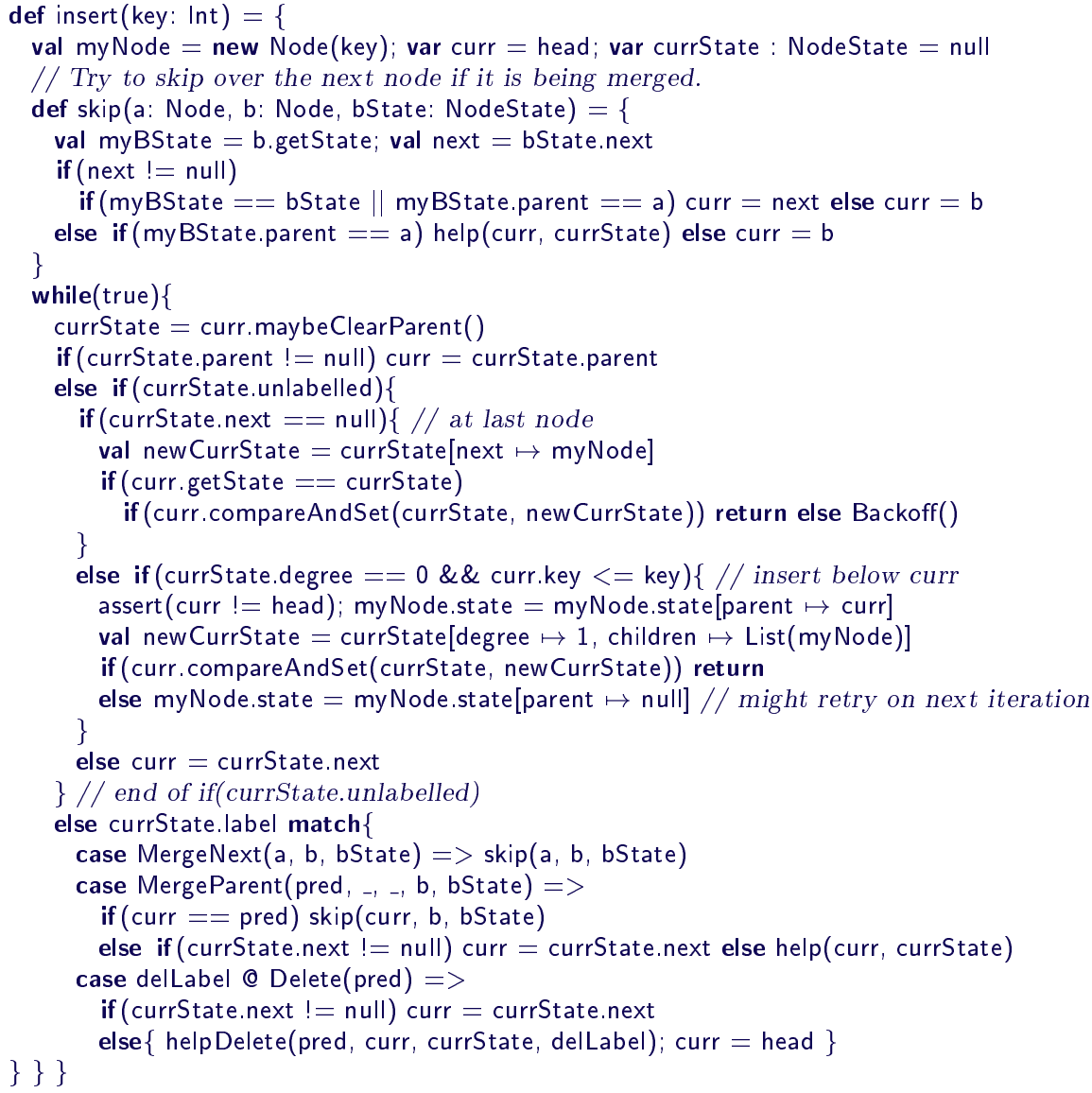

Figure 12: The insert function.

It is possible that the traversal reaches a node with a non-null parent (line 12): another thread might have merged curr below another node, or partially deleted curr's parent; in this case, the parent reference is followed.

If the end of the root list is reached, and the last node is unlabelled, the operation tries to append the new node after it (line 17). If the appending CAS is unsuccessful, this thread spins, using a binary back-off, to avoid creating memory conflicts. If the last node has a label, this thread helps with the corresponding operation.

If the traversal reaches an unlabelled root node of degree 0 whose key is no larger than $\mathrm{k}$ (line 19), the operation tries to insert the new node below it.

Otherwise, the next reference is normally followed to continue the traverse. However, if the current node has a MergeNext label (or a MergeParent label where the following node is the one being merged) then it is often advantageous to skip over the next node, which is about to be merged below another (similarly to as 
in advance, Section 5): indeed, if the b update has occurred, this is necessary in some circumstances to avoid getting stuck in a loop, and so to ensure the implementation is lock-free. This is implemented using the function skip: the b-node of the merge is skipped over, unless the merge has been interfered with and so can't complete.

A small enhancement is that if the last root is involved in another operation, it can be more efficient not to help that operation, but instead to restart the traversal, in the hope the operation completes anyway: our approach is to restart the first two times, and subsequently to restart with some probability; informal experiments suggest a probability of $\frac{7}{8}$ works well.

We seek to prove that the new node is correctly inserted, either after the last root, or as the child of a root. We need some additional results.

Definition 15. We say that a node is new if it has been created by the insert function but not yet added to the heap.

Invariant 6. Suppose node $n$ does not have a Delete label and is not new. Then $n$ is a root node if and only if n.parent is null or points to a decoupled node.

Lemma 16. If a node $n$ has null next, parent and label fields, and $n$ is not new, then it is the final root.

Proposition 17. If insert terminates, it correctly inserts key.

Proof: Suppose insert inserts the new node at line 17 of Figure 12, after curr. Then by Lemma 16, curr was the last root node. Alternatively, suppose insert inserts the new node at line 22, as the child of curr. Then by Invariant 6 , curr was a root, which has now become the root of a tree of degree 1 . We linearize the operation at the point of the successful CAS in each case.

In order to prove lock-freedom, we aim to prove that insert terminates assuming a bounded amount of interference. Our approach is to identify a rank, from a well ordered set, that is reduced by each iteration (assuming no interference). The rank is composed of several parts, which we introduce below, together with additional results to show that the rank is indeed decreased.

If the traversal just traverses the root list, then it is clearly bounded (cf. Invariant 5 ). However, things are complicated by the fact that if a node $d$ has been decoupled but not deleted, the traversal can reach $d$ 's first child, and then follow that child's parent field to $d$. Nevertheless, the following quantity is reduced by each such step.

Definition 18. Consider a state of a binomial heap. We define the next rank of a node $n$, written nextRank $(n)$, as follows:

- For convenience, we define nextRank $($ null $)=0$.

- Suppose $n$ is a root node, and $n^{\prime}$ is the following root, or $n^{\prime}=$ null if $n$ is the final root; then nextRank $(n)=2+\operatorname{nextRank}\left(n^{\prime}\right)$; so nextRank $(n)$ is twice the number of root nodes from $n$ onwards. 
- If $n$ is decoupled but not deleted, then we define nextRank $(n)=1+$ nextRank(n.next).

- Otherwise, we define nextRank $(n)=\infty$.

Lemma 19. Suppose node $d$ is decoupled but not fully deleted, and has at least one child, and suppose $d$.next $\neq$ null. Then $d$.next is a root node.

Informally, $d$ 's last child points to $d$.next at the point of decoupling, and continues to do so until after $d$ is fully deleted; so $d$.next remains a root during this period.

Lemma 20. Suppose $n$ is a root node, and $p=n$.parent is decoupled, but not fully deleted. Then nextRank $(p)<\operatorname{nextRank}(n)$.

The traversal might reach a node that is subsequently deleted. From there, it follows the next reference, to reach a node that might also have been subsequently deleted. We need to show that this cannot continue indefinitely, assuming that eventually no more nodes are deleted. We need the following definition.

Definition 21. Consider a particular execution and a particular point in that execution. Suppose each of nodes $n_{0}, n_{1}, \ldots, n_{k-1}$ either has no children and has been decoupled, or has been fully deleted; and suppose their pred update steps were performed in the above order. Define the deletion rank of a node $n$ to be

$$
\begin{array}{cl}
k-i & \text { if } n=n_{i}, \text { for } i=0, \ldots, k-1, \\
0 & \text { if } n \neq n_{0}, n_{1}, \ldots, n_{k-1} .
\end{array}
$$

Note that nodes that have earlier pred updates have larger deletion indices.

Lemma 22. Suppose node d either has no children and has been decoupled, or has been fully deleted. Then d.next has a smaller deletion rank.

Definition 23. We define the depth of a node $n$ to be the number of parent references that can be followed to reach a root node; if $n$ is decoupled or fully deleted, we define its depth to be 0 . Note that this depth is finite, by Invariant 3.

Lemma 24. Consider an invocation of insert, during which other threads make a finite number of updates to nodes. Then insert terminates in a finite number of steps, outside of helping.

Proof: (sketch) Define the rank of the current node curr to be a triple:

(the deletion rank of curr, the depth of curr, the next rank of curr).

We order ranks lexicographically; note this is a well founded order.

The above lemmas can be used to show that, under the assumptions of this proposition, the rank is reduced by each iteration of the loop of insert except a helping iteration or the final iteration.

Lemma 2 and Proposition 14 show that only a finite number of attempts to help are necessary for each node. Further, there are initially a finite number of nodes with labels, and no new nodes get labelled, by assumption; hence only a finite number of helping iterations are necessary in total. 


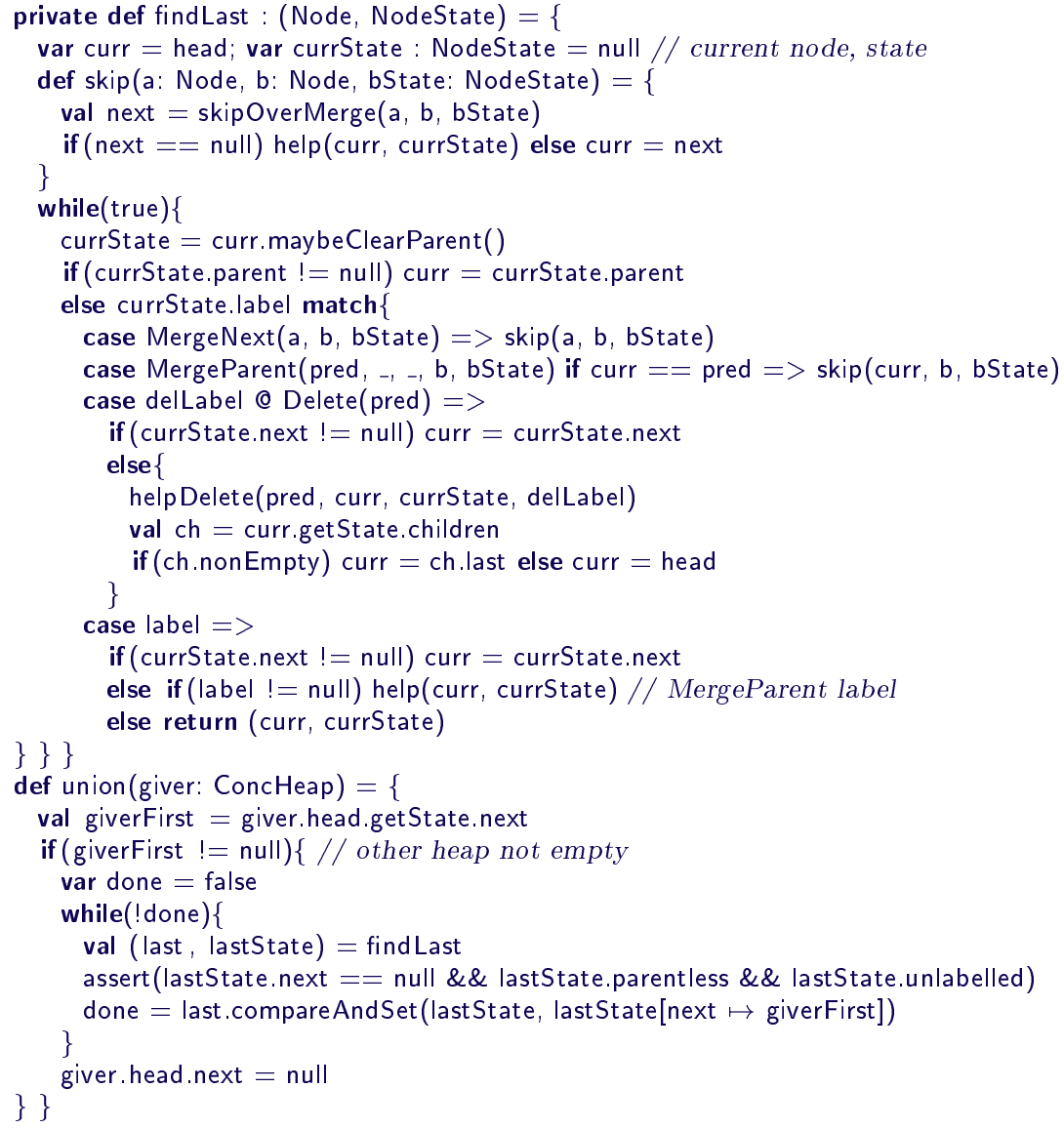

Figure 13: The findLast and union functions.

\section{7. union}

In this section we present a simple implementation of the union operation. Recall that we assume here that there are no concurrent operations on the giving heap (denoted "giver", below), although we do allow concurrent operations on the receiving heap (the heap of the operation); we relax this assumption in Section 11. We also assume that the two heaps are distinct.

The union operation works by locating the last root node of the current heap, and updating its next pointer to reference the first (non-header) node of giver. Most of the work is done by the findLast function (Figure 13), which finds the last root and its state, also helping with the operation of any label on the last root. The traversal is almost identical to that in insert (except without trying to insert nodes). If it helps with the deletion of the last root, it tries to continue from that node's last child, if there is one, since that node is likely to be near 
the end of the root list; otherwise it restarts the traversal. If it helps with a merge, it continues from the same node.

The union function is then straightforward. If the giving heap is empty, there is nothing to do. Otherwise it finds the last root of the heap, and tries to update it so its next pointer points to the first proper root of the other heap; we call this the joining $C A S$. It then clears the other heap. If the joining CAS is unsuccessful, it retries. The following results are proved in a similar way to corresponding results for insert.

Lemma 25. The union function operates correctly, assuming there are no concurrent updates on its argument giver.

Lemma 26. Consider an execution, during which, from some time on, no other thread performs any update to any node. Then union terminates in a finite number of steps, outside of helping.

\section{Minimum}

We now discuss the minimum function, which finds the smallest key in the heap. It traverses the root list, in a similar style to findPred.

If the minimum key has been marked for deletion before the traversal completes, but has not been fully deleted, it can still be returned by minimum. We justify correctness, i.e. linearizability, in Section 13. The fact that the deletion has not been completed means that the relevant deleteMin function has not yet returned: the linearizability proof makes use of this. However, our proof of linearizability requires that the node should not have been marked before the call to minimum: we use the timestamps in the Delete labels to test this ${ }^{9}$. We assume that the timestamping mechanism is correct in the following sense: suppose one thread generates a timestamp $t s_{1}$ and writes it to shared memory; and a second thread later generates a second timestamp $t s_{2}$; then $t s_{1} \leq t s_{2}$ (ignoring overflow).

It would not (in general) be correct to return a key found during the traversal if the corresponding node is fully deleted before the traversal is finished, even if the node had not been marked for deletion before the call to minimum began. Consider a heap that initially contains the keys 2 and 3 , in that or-

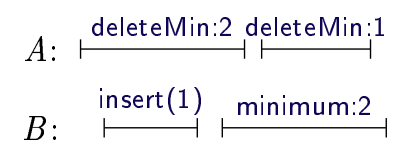
der, and consider the execution illustrated by the timeline to the right, and explained below.

1. Thread A starts a deleteMin, identifies 2 as the minimum key, and stalls;

2. Thread B inserts key 1 (at the end of the root list);

3. Thread B calls minimum, starts a traversal, sees the key 2, advances to 3 , but then stalls;

\footnotetext{
${ }^{9}$ An alternative would be to use a shared counter as a form of logical timestamp.
} 
4. Thread A resumes, fully deletes the key 2, and returns;

5. Thread A performs a deleteMin, and deletes 1;

6 . Thread B resumes, completes the traversal, and returns 2 .

The above is not linearizable: at no point during the execution of minimum is 2 the minimum key.

It can be advantageous, when traversing the root list, not to keep track of just a single smallest node ${ }^{10}$, but instead to keep track of the $m$ smallest nodes for some $m>1$. If the smallest node is fully deleted before the traverse finishes, we can instead consider the next smallest node. This will be even more the case in the deleteMin operation (Section 9): when the deleteMin finishes the traversal, there is a fairly high likelihood that the smallest node has already been marked for deletion by another deleteMin operation; in this case, it can try the next smallest node.

The minimum function traverses the list. It keeps track of the smallest nodes that it has seen, up to a maximum of numMinsMinimum such, and discarding others. If, at the end of the traversal, the smallest node minNode has been deleted, then minimum could return the next smallest node found during the traversal, or one of minNode's children, whichever is smaller. This requires some care. If more nodes were inserted after the initial traversal finished, but before minNode was found to be deleted, then we need to take those additional nodes into account to ensure linearizability. We therefore resume the traversal from the node that was previously the last node in the root list.

We use a subsidiary class Min- class MinList[N](numMins: Int, getKey: $N=>\operatorname{lnt})\{$ List, outlined to the right, to store these minimal nodes. In fact, this class is polymorphic, to allow it to be re-used in the traversal of deleteMin. It stores up to num Mins elements of type $\mathrm{N}$, namely those that are minimal under the getKey function. In minimum, we instantiate $\mathrm{N}$ with Node, and getKey with the function that returns a node's key.

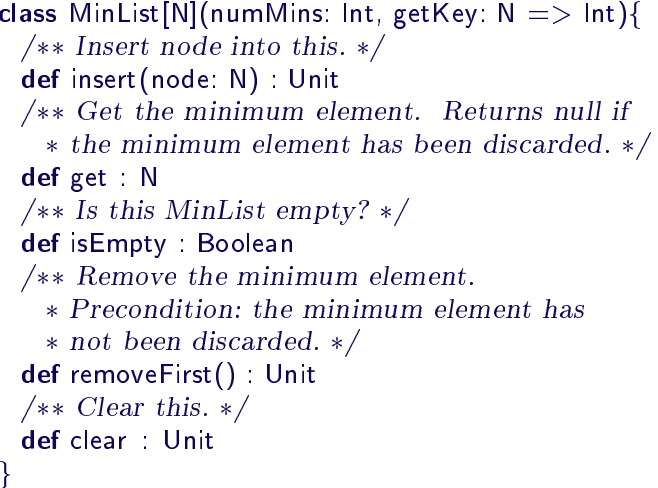

We omit the implementation since it is straightforward (and sequential). Note that the insert operation starts to discard nodes once the limit of numMins is reached. A critical point, however, is the following: if previously a node $n$ was discarded because the limit had been reached, and subsequently a node was removed from the MinList (creating a space) but found to be deleted, and then another node is inserted (either a child of the deleted node, or a node found during the resumed traversal), then we should not store such nodes with a larger key than a node that was previously discarded; otherwise, if all the nodes found

${ }^{10}$ We use terms such as "smallest node" to mean the node with the smallest key. 


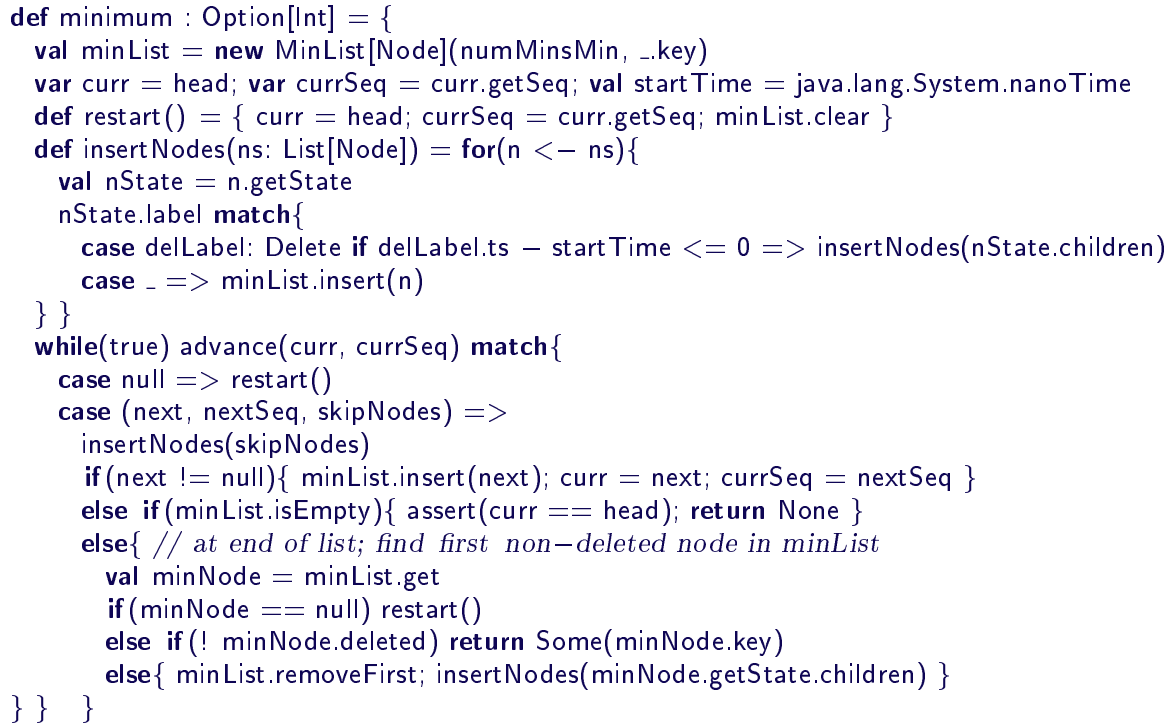

Figure 14: The minimum function.

during the initial traversal are subsequently found to be deleted, we could incorrectly return a key that was larger than one in a discarded node. We therefore record the minimal key that has been discarded, and do not subsequently store a node with a larger key. If a node has been discarded and no other node is stored, a call to get returns null; the traversal has to restart in this case.

The critical properties of MinList are captured by the following lemma.

Lemma 27. If get returns a non-null node, it is the smallest node that was added to the MinList since the last call of clear and has not been removed. isEmpty returns true if and only if every node that was added to the MinList since the last call of clear has also been removed.

The minimum function (Figure 14) traverses the list, keeping track of the minimal nodes encountered in minList. At each step, it uses advance to obtain the next node and its sequence number, and maybe some nodes skipped over. If advance returns null, minimum restarts. Otherwise, it inserts relevant nodes into minList; if a node skipped over is marked for deletion, it is inserted only if the marking was after the start of the traversal; otherwise the children are recursively inserted.

When the traversal reaches the end of the root list, if minList is empty, then the heap was empty, so minimum returns None (line 16). If the call to minList.get returns null, then the minimum node has been discarded, so the traversal has to restart (line 19). Otherwise, if the minimal node minNode has not been fully deleted, minimum returns minNode's key. If minNode has been fully deleted, minNode is replaced by its children in minList. 
We want to prove that all the nodes in the heap are represented by the nodes added to minList. More precisely, each node in the heap is a descendant of one of those nodes.

Definition 28. We define the children of node $n$ to be n.children, unless $n$ is the a node of a merge, and the $b$ update has taken place, in which case we also include $b$ within the children. We define the descendants of a node $n$ to be $n$ itself, its children, its children's children, and so on. We lift descendants to sets of nodes, pointwise. We write nodes $U p T o(r)$ for the descendants of the roots up to $r$, i.e. descendants (rootsUpTo(r)) (recall Definition 10); so nodesUpTo(null) represents all the nodes in the heap. We write unmarked NodesUpTo $(r)$ for the nodes in nodesUpTo(r) that do not have a Delete label.

Part of the invariant of the traversal is that the unmarked nodes up to the current position are a subset of the descendants of nodes added to minList. The following lemma identifies circumstances under which this property is maintained by actions of other threads.

Lemma 29. Let $S$ be a set of nodes. Suppose root node $r$ does not have a Delete label, and suppose unmarkedNodesUpTo $(r) \subseteq$ descendants $(S)$. Then the above relation is maintained by each step of an operation that does not change the sequence number of $r$, other than a giving union.

Proof: (Sketch; see [6] for full proof.) The only additions to unmarked Nodes$U p T o(r)$ are as the result of a merge or an insertion below an existing unmarked root $r_{1}$ that is earlier than $r$. But $r_{1}$ will be in descendents $(S)$; so the same nodes are added to both sides of the above equation.

The following proposition gives a correctness condition for minimum. In Section 13, we use this result to prove linearizability. Below, by "the end of the traversal" we mean, more precisely, when the final node's state was read in advance (line 4 of Figure 10), i.e., the state whose next field was subsequently found to be null.

Proposition 30. If minimum returns a key $k$ then, at the end of the traversal, the corresponding node was not deleted, and there was no smaller unmarked node in the heap. If minimum returns None, then at some point during the call, ${ }^{11}$ the heap had no unmarked node.

Proof: As in Lemma 12, if the sequence number of curr has not changed, then curr is a root node, and does not have a Delete label.

Let $S$ be the set of nodes that have been added to minList, but not removed, since the last time it was cleared. We show that the following is invariant: if the sequence number of curr has not changed, then

$$
\text { unmarkedNodesUpTo(curr) } \subseteq \text { descendants }(S) .
$$

\footnotetext{
${ }^{11}$ In fact, this property holds at the end of the traversal; however, in Section 11 we will have the slightly weaker property stated here; and this weaker property is enough for our proof of linearizability.
} 
This is true initially, and is re-established whenever the traversal restarts.

Consider a call to advance that returns a result (next, nextSeq, skipNodes), in an iteration that neither returns a value nor restarts the traversal. By part 2 of Lemma 9 ,

$$
\begin{aligned}
& \quad \text { nodes } U p T o(\text { next })= \\
& \quad \text { nodes } U p T o(\text { curr }) \cup \text { descendants }(\text { skipNodes }) \cup \text { descendants }(\text { next }) .
\end{aligned}
$$

The additions to minList have the effect of adding (at least) the unmarked nodes of descendants(skipNodes) $\cup$ descendants(next) to the right-hand side of equation (1), thereby maintaining this equation when curr is set to next.

If, at line 21, minNode is removed from minList, and its children added, then this removes just minNode from the right-hand side of (1). But minNode was marked for deletion, so this maintains the property.

Equation (1) is maintained by steps of other threads, by Lemma 29. Hence this invariant is maintained.

Now suppose advance returns Some(minNode.key) at line 20 . Then by Lemma 27, this value was minimal in $S$. Hence, by equation (1) and Invariant 1 , there was no smaller unmarked node in the heap. Further minNode is not deleted.

Finally suppose advance returns None at line 16 . Then minList was empty, so by Lemma $27, S=\{\}$. Hence, by equation (1), there is no unmarked key in the heap.

The following lemma will prove useful in proving linearizability; it follows from the use of timestamps, and the fact that we assume that a deleteMin operation can not be concurrent to a giving union.

Lemma 31. If a minimum operation returns the key from node $n$, then $n$ was not marked for deletion when the operation started its traversal; further, it was not marked for deletion while in a different heap.

The following lemma captures a liveness property for minimum.

Lemma 32. Suppose a call to minimum occurs such that, from some point on, no other thread makes any update to any node. Then the call returns after a finite number of steps.

\section{9. deleteMin}

We now describe the traversal used within deleteMin; see Figure 15. We need to identify the minimum node and its predecessor. The code is very similar to the code for minimum. minList stores up to numMinsDelMin pairs of the form $(p, n)$, where $n$ is a node encountered during the traversal, and $p$ is its expected predecessor; these pairs are ordered by the key of the $n$ components. Our experiments suggest a value of around 16 works well for numMinsDelMin; this roughly doubles throughput over recording a single minimum. (We expect that 


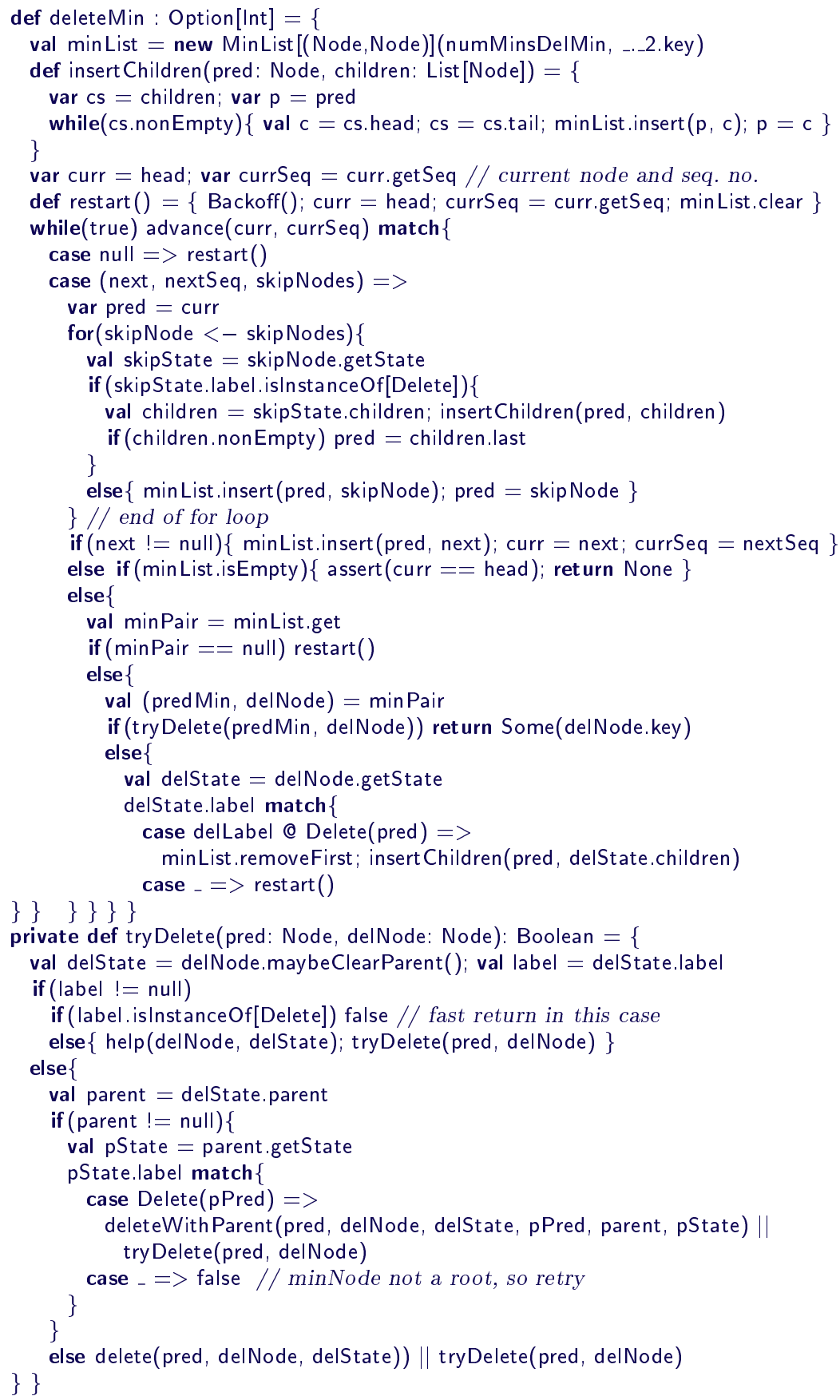

Figure 15: The deleteMin and tryDelete functions 
with more threads, a higher value would work better: we leave experiments investigating this as future work.)

While traversing, deleteMin inserts each node and its predecessor into minList (line 21). Similarly, it iterates through the nodes skipped over, and through the children of such nodes marked for deletion (via the helper function insertChildren), and inserts each and its predecessor into minList. (Recall that the correctness of the predecessor is not essential for the subsequent deletion, but is a useful optimisation.)

When it reaches the end of the list, if minList is empty, then the heap is empty, so None is returned. If the call to minList.get returns null, then the minimum node has been discarded, so the traversal restarts. Otherwise, it extracts the minimal node minNode and its expected predecessor predMin from minList. It passes these to the function tryDelete (see below), which carries out various checks to test whether the deletion might succeed, maybe helping with other operations, and then tries to delete the node itself. If this is successful, minNode's key is returned. If the deletion failed because minNode has been marked for deletion by another thread, minNode is replaced in minList by its children. If the deletion failed for some other reason, it is necessary to restart the traversal.

The most likely cause of the deletion failing is if another thread has already claimed the node for deletion. tryDelete therefore tests this first (line 39). If delNode has a label other than a Delete, then tryDelete helps with that operation (line 40), and then retries. If delNode was found as the child of a node without a Delete label, then it must have been merged below another node since it was encountered in the traversal; it cannot now be deleted so tryDelete fails. Otherwise, it calls either delete or deleteWithParent, as appropriate, to try to delete the node, recursing if unsuccessful.

The proof of the following result is nearly identical to that of Proposition 30.

Proposition 33. If deleteMin returns Some $(k)$ then $k$ was a minimal unmarked key when the traversal ended. If deleteMin returns None then the heap had no unmarked nodes at some point during the call.

We now give a liveness result for deleteMin.

Lemma 34. Suppose a call to deleteMin occurs such that, from some point on, no other thread makes an update to any node. Then the call returns after a finite number of steps, outside of helping.

\section{Tidying}

In this section we sketch when and how threads attempt to merge trees. We implement a function tidy() that performs this merging. There is a trade-off concerning how often tidy is called. Calling it more often leads to a shorter root list, which makes traversing faster. However, tidying takes time, and also risks interfering with other operations. We therefore arrange for a thread to call tidy only occasionally. More precisely, we use a shared integer counter 
opCount, ${ }^{12}$ and an integer parameter tidyRatio, indicating how frequently a thread should perform tidying. Every time a thread completes an insertion or deletion, it increments opCount; if it is then a multiple of tidyRatio, it calls tidy. Our experiments suggests a value of between 2 and 8 for tidyRatio works best. In addition, when a thread completes a union operation, it calls tidy directly (since it is likely that this operation has significantly lengthened the root list).

The tidy function traverses the root list, in a similar way to insert. It maintains an array buckets of pairs of nodes: if buckets $(d)$ holds a pair ( $p r e d, n)$ then, when these nodes were encountered earlier, $n$ was a node of degree $d$ and had predecessor pred, or pred was likely to become the predecessor of $n$ after some pending operation completed. At each step in the traversal, tidy either tries merging the current node with a node encountered earlier, or stores the appropriate pair in buckets. As an optimisation, we avoid performing a merge of the last root node or a singleton root: insert operations use each of these, so merging such a node can disrupt insertions. We omit the code, since it is straightforward.

Lemma 35. If tidy runs, and, from some point on, no changes are made to the root list by other threads, then it performs a finite number of steps.

\section{Allowing concurrent unions}

In this section we explain how to re-implement the union operation so as to allow it to run concurrently with other operations on the giving heap. We start by highlighting some of the difficulties, so as to motivate parts of our design; in particular, we show what could go wrong with a less careful implementation.

Suppose a call to minimum occurs on heap $h_{g}$ concurrently to a call to $h_{r}$.union $\left(h_{g}\right)$. Suppose, further, that immediately after the two heaps are joined, a new key $k$ is added to $h_{r}$. Then the traversal of minimum may encounter $k$, and incorrectly return it, even though it is not part of $h_{g}$. We avoid this problem as follows: when the traversal finishes, the thread detects whether a giving union on $h_{g}$ has been linearized during the traversal; if so, minimum returns None, and the call can be linearized immediately after the linearization point of union, at which point the heap was indeed empty. The main mechanism we use to detect whether such a union has been linearized is to equip each heap with an integer variable epoch which counts the number of such unions so far.

A similar problem arises with deleteMin. However, the above approach is not sufficient. Consider the following behaviour. (1) Thread $A$ calls deleteMin on heap $h_{g}$, identifies $k$ as the minimum key, checks that no concurrent union has occurred, but then is suspended; (2) thread $B$ performs $h_{r}$.union $\left(h_{g}\right) ;(3)$ thread $C$ calls minimum on $h_{r}$, and returns $k$; (4) thread $A$ resumes and deletes $k$. This behaviour is not linearizable because of the delay between the check in step 1 and the update in step 4: in effect, thread $A$ has deleted $k$ from $h_{r}$ rather

\footnotetext{
${ }^{12}$ An alternative would be to use thread-local counters.
} 
than $h_{g}$. To avoid this, we ensure that no giving union occurs concurrently to the delNode labelling of a deletion. We explain the mechanism below.

Further, suppose deleteMin labels a node delMin for deletion, but has to call findPred to locate the predecessor of delMin. If delNode has been moved to a different heap in the meantime, then obviously findPred has to search in that different heap. We therefore need a mechanism for locating the current heap that a node is in. Also, if findPred fails to find delNode, there are subtleties in deciding whether it has really been decoupled so can be marked as fully deleted.

Similarly, without care, unions can interfere with insertions on the giving heap, so that the insertion happens after the union, and so effectively inserts into the receiving heap.

Finally, two concurrent unions on the same heap could interfere with one another. Consider two threads performing $h_{1}$.union $\left(h_{2}\right)$ and $h_{2}$.union $\left(h_{3}\right)$. Suppose the former joins its two heaps first. Then, without suitable care, the latter thread could join $h_{3}$ onto the combined heap, $h_{1}$, rather than $h_{2}$. Similarly, if we have a cycle of unions, for example if $h_{1}=h_{3}$, above, then this could create a circular root list. We prevent this by labelling both heaps, more precisely their head nodes, at the start of the union, with UnionGiver and UnionReceiver labels. This prevents concurrent unions affecting the same heap. However, we clearly need a mechanism to detect a cycle of such labelling, which could create a deadlock.

Similarly, to prevent a giving union concurrently to the critical step of an insertion or deletion (as justified above), we add a special label, which we call a heap label to the head node during that critical step. However, we can allow the critical steps of insertions or deletions to occur concurrently to one another, or concurrently to the heap being the receiving heap of a union. We therefore allow the head node to have a list of heap labels and at most one UnionReceiver label, allowing these to occur concurrently, but not concurrently to a giving union; these are encapsulated in a HeapLabelList.

Recall that we need a mechanism for enabling a thread to identify the current heap that a node is in; we want to be able to update this mechanism efficiently when a union happens. We use a technique based upon the union-find data structure [16]. Each heap has a heap indirection object. Each heap indirection contains a reference to either its heap or another heap indirection object. When a node (other than the dummy header) is added to a heap, it is given a reference to the corresponding heap indirection. Thus following a path of such references leads to the corresponding heap, except temporarily during a union. Each union operation updates the heap indirections to re-establish this property.

We now outline how union proceeds. Figure 16 illustrates the operation.

1. If the giving heap is empty, the union operation is equivalent to a no-op, and so returns immediately. Otherwise, it labels the head node of the giving heap with a UnionGiver label. This has a side effect of ensuring that the first proper node remains as such. The UnionGiver label acts as a convenient place to store information about the state of the union. 


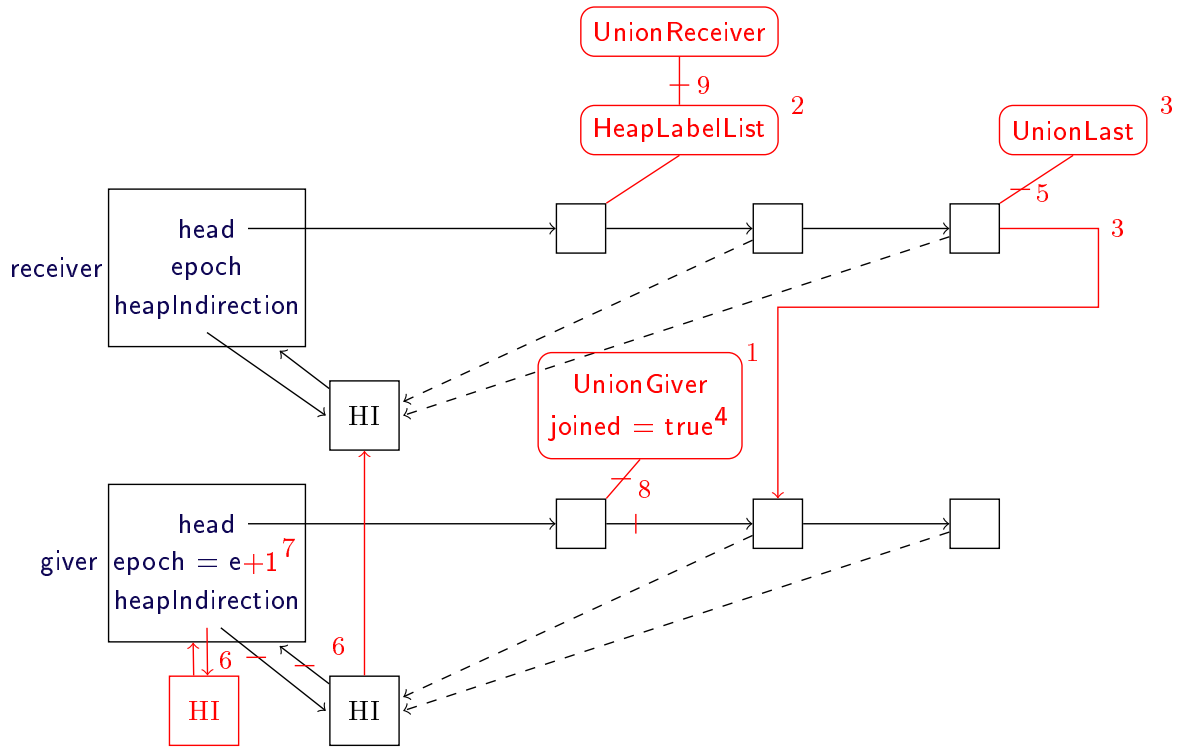

Figure 16: Illustration of union. Drawing conventions extend those in Figure 2 as follows: the large boxes on the left are heap objects; the boxes labelled "HI" are heap indirections; dashed lines from a node to a heap indirection represents a path via zero of more intermediate heap indirections.

2. It tries to label the head node of the receiving heap with a UnionReceiver label, either adding a HeapLabelList to hold it, or using an existing HeapLabelList. If this addition is blocked because that node already has a UnionGiver label, it tests whether there is a cycle of such labelled nodes, and if so removes one such label and replaces it with the corresponding UnionReceiver label; the union operation corresponding to the replaced label then restarts.

3. It finds the last node in the receiving heap, and updates its next pointer to point to the first proper node of the giving heap. We call this the joining $C A S$. It is the linearization point of the union: we consider the nodes to be transferred from the giving heap to the receiving heap at this point. If the receiving heap is non-empty, it simultaneously labels that last node with a UnionLast label; this allows other threads to detect that the linearization point has passed. (If the receiving heap is empty, the last node is the head, and retains its HeapLabelList label.)

4. It updates the UnionGiver label to indicate that the joining CAS has occurred.

5. If the receiving heap was non-empty, it removes the UnionLast label from the former last node of the receiving heap.

6. It sets the giving heap's heap indirection to reference that of the receiving heap; hence following the path of heap indirections from a former node of the giving heap leads to the receiving heap, as required. The giving heap 
receives a new heap indirection.

7. It increments the giving heap's epoch.

8. It atomically sets the giving heap's head's next reference to null and removes the UnionGiver label.

9. It marks the UnionReceiver label as complete, and removes it from the HeapLabelList.

As with previous operations, other threads might help with steps after the first; and it might be necessary to help other operations that block the union.

Each UnionGiver and HeapLabelList applies to the whole heap, rather than to an individual node. In a previous version, we made them attributes of the heap, rather than the head node. However, it also proved necessary to label the head of the giving heap, to prevent the reference to the first node from changing before the giving CAS: it simplified things to combine these two labels.

We augment the state of each heap with an epoch and the heap indirection.

private val epoch $=$ new AtomicLong $(0)$

private val heaplndirection $=$ new AtomicReference(new Heaplndirection(this))

We describe the Heaplndirection class later.

In the remainder of this section, we describe the union operation in more detail: we start by outlining HeapLabelLists; in the following four subsections, we describe the different phases of the union; we then describe how other threads help with the union, and give correctness results. In the next section we describe how other operations have to be adapted to allow concurrent giving unions.

\subsection{HeapLabelLists}

We now sketch the implementation of HeapLabelLists. Recall that each HeapLabelList holds a list of HeapLabels, and at most one UnionReceiverLabel.

HeapLabels are defined in Figure 17. Each contains a next reference to allow them to be formed into a linked list, and a boolean done, which is set when the relevant steps are completed. We explain the subclasses when we explain how the critical steps are implemented. Figure 17 also defines the UnionReceiver labels. These simply contain a reference to the giving heap, and a boolean indicating whether the union has been completed.

Figure 18 gives the interface of the HeapLabelList class. We only sketch the implementation, in the interests of brevity, since it is fairly straightforward. The HeapLabels are arranged in a linked list, closely following the lock-free queue of Michael and Scott [17]. The head of this list and the current UnionReceiver (possibly null) are encapsulated into a pair to make them atomically updateable. The two add operations add a HeapLabel or a UnionReceiver, respectively; the latter might need to help to complete the current UnionReceiver, first, but takes no action if ur is the current UnionReceiver, or if ur.done is true. The close operation helps to complete all the current operations, and then removes the HeapLabelList from the heap's head. If an add operation finds that the HeapLabelList is being closed, it helps with that and returns false to indicate a failure. The remove operation removes the current UnionReceiver; this happens after it is marked as done. 


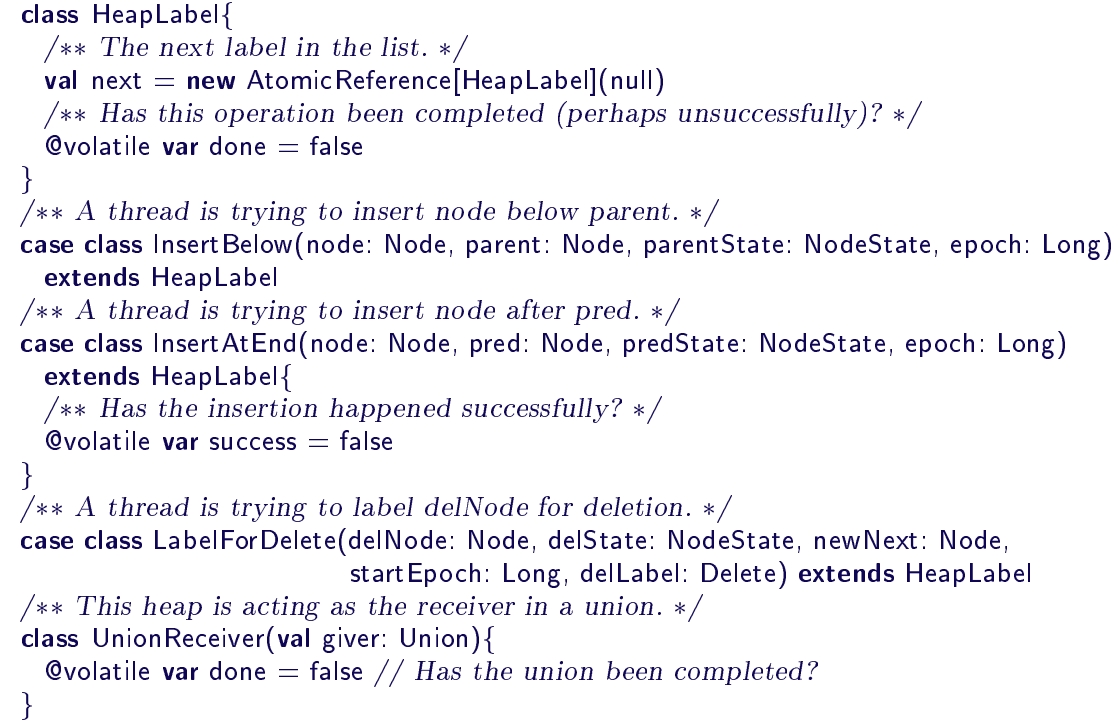

Figure 17: HeapLabels.

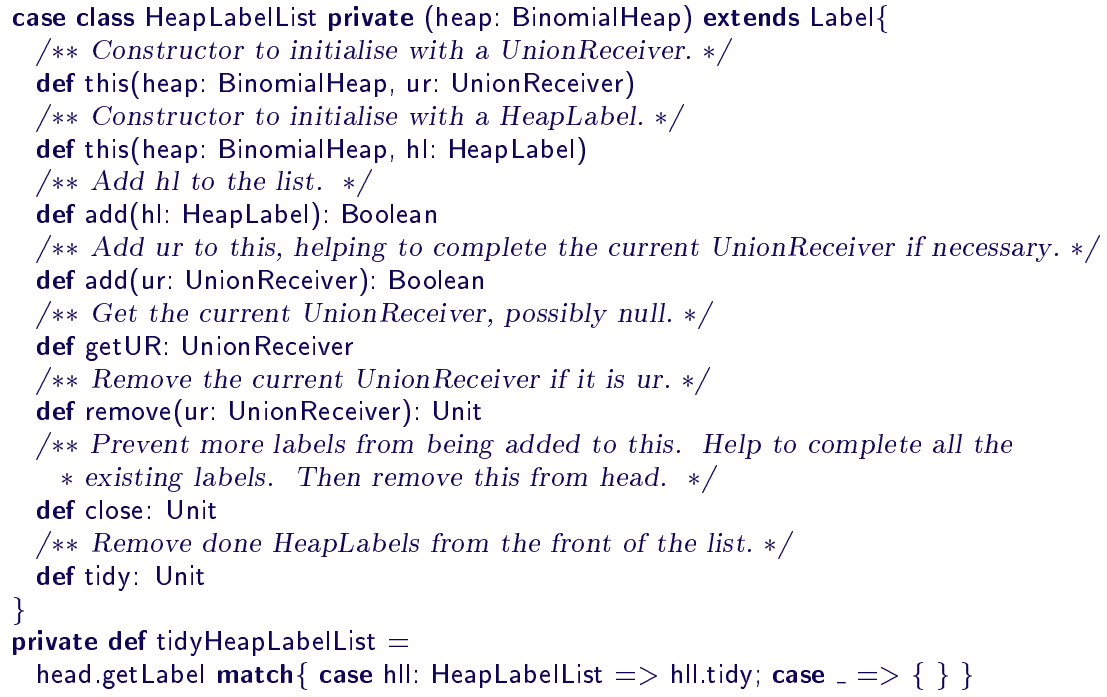

Figure 18: Interface of the HeapLabelList class, and the tidyHeapLabelList function.

When an operation is completed, the label's done label is set; the function tidy (as an optimization) removes such labels from the list; the function tidyHeapLabelList (in the BinomialHeap class) invokes tidy on the current HeapLabelList, if any. 


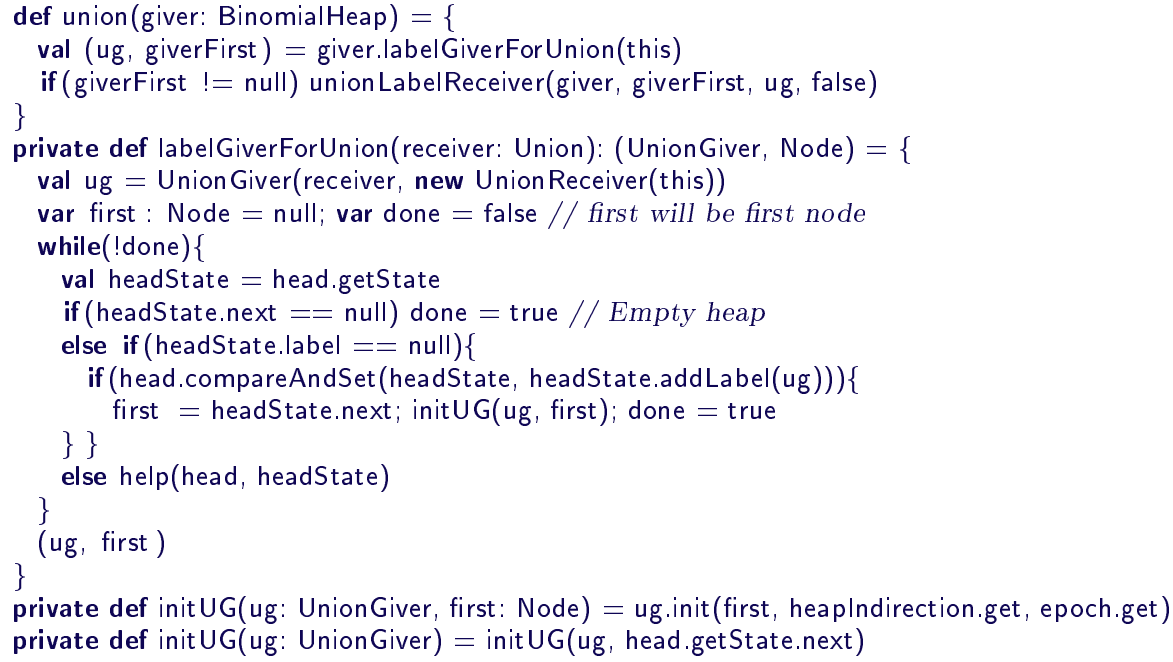

Figure 19: The union, labelGiverForUnion and initUG functions.

Rule 4. A node with a HeapLabelList label can be updated only as follows.

- To remove the label if all the operations in it are complete;

- To update its next reference from null, either to insert a new node, or as the joining CAS of a union;

- To perform the pred update of the deletion of the following node $n$; however if the HeapLabelList contains a UnionReceiver, and the joining CAS has happened with $n$ as the first node of the giving heap, then the corresponding UnionGiver must first be updated to record that the CAS has happened (corresponding to step 4 of the union).

\subsection{The labelling of the giving heap}

Recall that the union operation operates on the receiving heap, and takes the giving heap as an argument. However, some steps are performed on the giving heap.

The union operation starts by labelling the giving heap's head node, via the labelGiverForUnion function (Figure 19), which returns the label added and the first proper node of the giving heap. It first prepares the UnionGiver label for the giving heap, and the UnionReceiver label for the receiving heap. Figures 20 and 24 give the UnionGiver class; the class encapsulates a lot of functionality, which we explain as we use it.

The labelGiverForUnion function tests whether the giving heap is empty; if so, it returns null as the first node of the heap, and the union function returns. Otherwise, if the head has a null label, it tries to add the UnionGiver label; if the head's label is not null, it helps with the corresponding operation, and re-tries. 


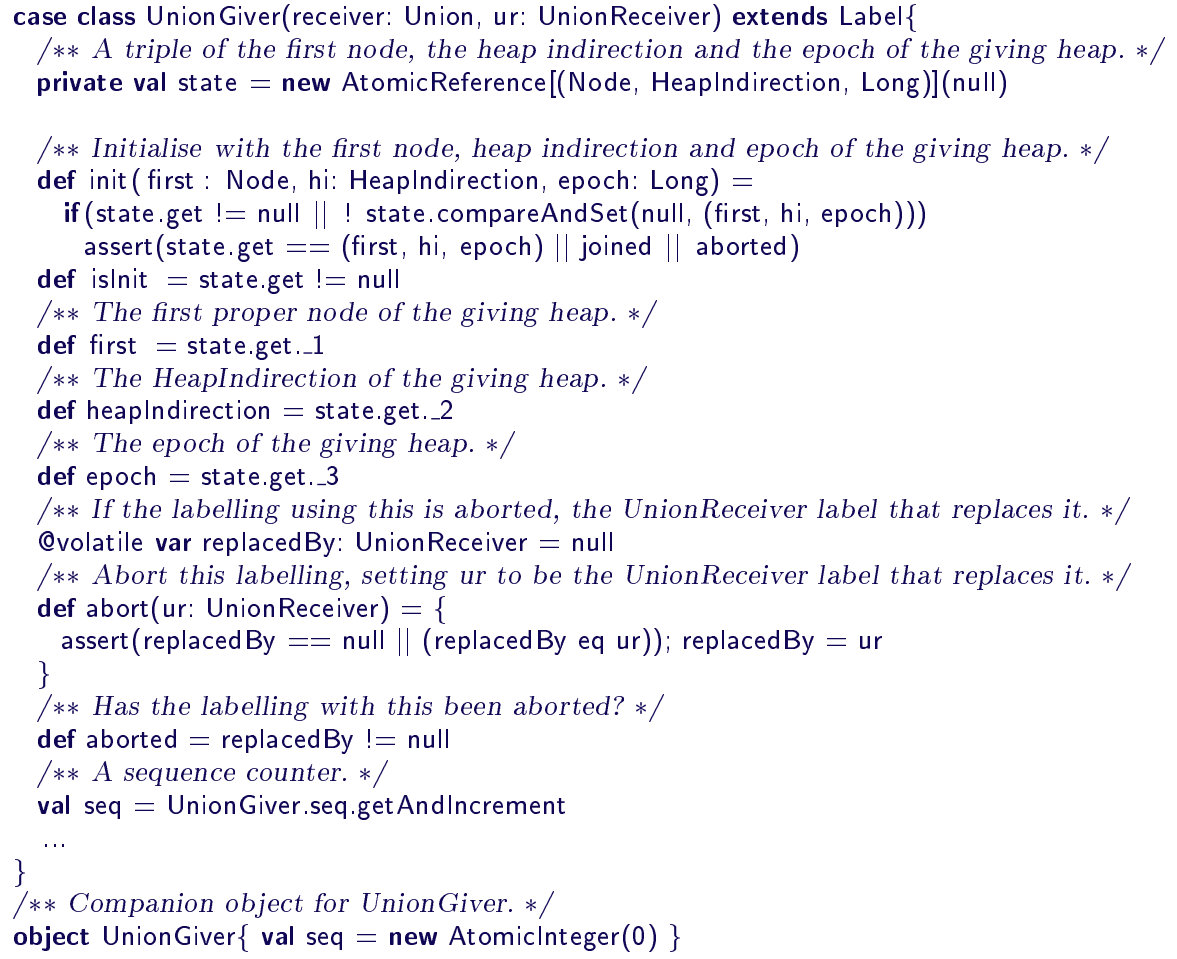

Figure 20: Part of the UnionGiver class, and the UnionGiver companion object.

It then initialises the UnionGiver to store the first node, the heap indirection and the epoch of the giving heap. It is important that this happens after the UnionGiver label is added to the header, to ensure the information is current. The information remains current until updated as part of this operation, or this labelling is backtracked, as captured by the following rule.

Rule 5. The heap indirection and epoch of a heap change only when that heap is labelled with a UnionGiver label (at steps 6 and 7 , respectively).

Another thread might help with the initialisation via one of the initUG functions; in each case, it provides consistent values until the joining CAS or the labelling is backtracked, as captured by the assertion within init.

Rule 6. A node with a UnionGiver label can be updated only as follows.

- To clear this label and its next reference as step 8 of the union; or

- To replace the label with a HeapLabelList containing a UnionReceiver label, to break a blocking loop (step 2). 


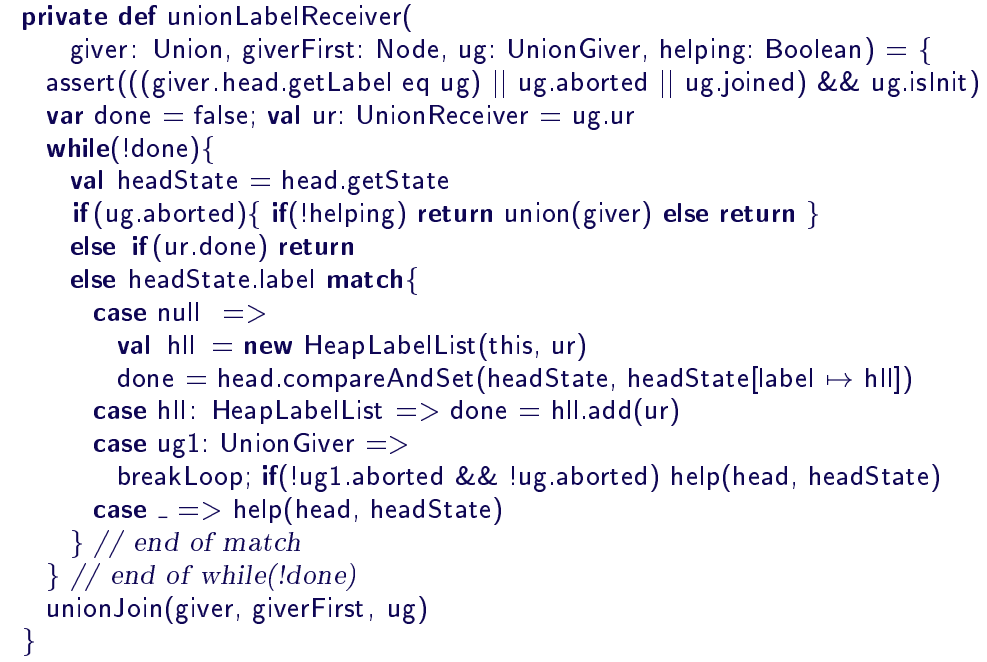

Figure 21: The union LabelReceiver function.

\subsection{The labelling of the receiving heap}

The unionLabelReceiver function (Figure 21) attempts to add the UnionReceiver label ur to the receiver's head node. However, it's possible that this node is already labelled with a UnionGiver label, blocking this union, and that there is a loop of such blocking; in this case, one of the UnionGiver labels is removed, aborting that previous labelling. The function assumes that the giving heap has already been labelled with ug, although the label might have been removed if it has been backtracked or the union completed, and ug has been initialised. The parameter helping is true for helping threads.

The normal operation is to add the UnionReceiver label ur to a new or existing HeapLabelList (lines 11 and 13). However, there are several corner cases.

If the UnionGiver label ug has been backtracked, as captured by its aborted function, then the union operation restarts, unless this thread is simply helping. If the union operation has been completed (by a helping thread), as captured by the done field on ur, then the function simply returns (this is necessary to avoid re-adding the label, which could lead to the union being done twice).

If the head node is labelled with another UnionGiver label ug1, then the function breakLoop (described below) searches for a loop of blocking unions, and backtracks one such label, aborting that labelling. If neither ug nor ug1 were aborted, this operation helps with ug1 and re-tries.

Finally, if the head node is labelled with some other label, then this is helped.

The code to detect blocking loops of union operations is in Figure 22. We say that heap $h$ is blocked by heap $h^{\prime}$ if $h$ has a UnionGiver $\left(h^{\prime}, u r\right.$ ) label (so the corresponding union operation seeks to label $h^{\prime}$ with $\left.u r\right)$, but $h^{\prime}$ also has a UnionGiver label. The function follows the path of such blocking, recording the 


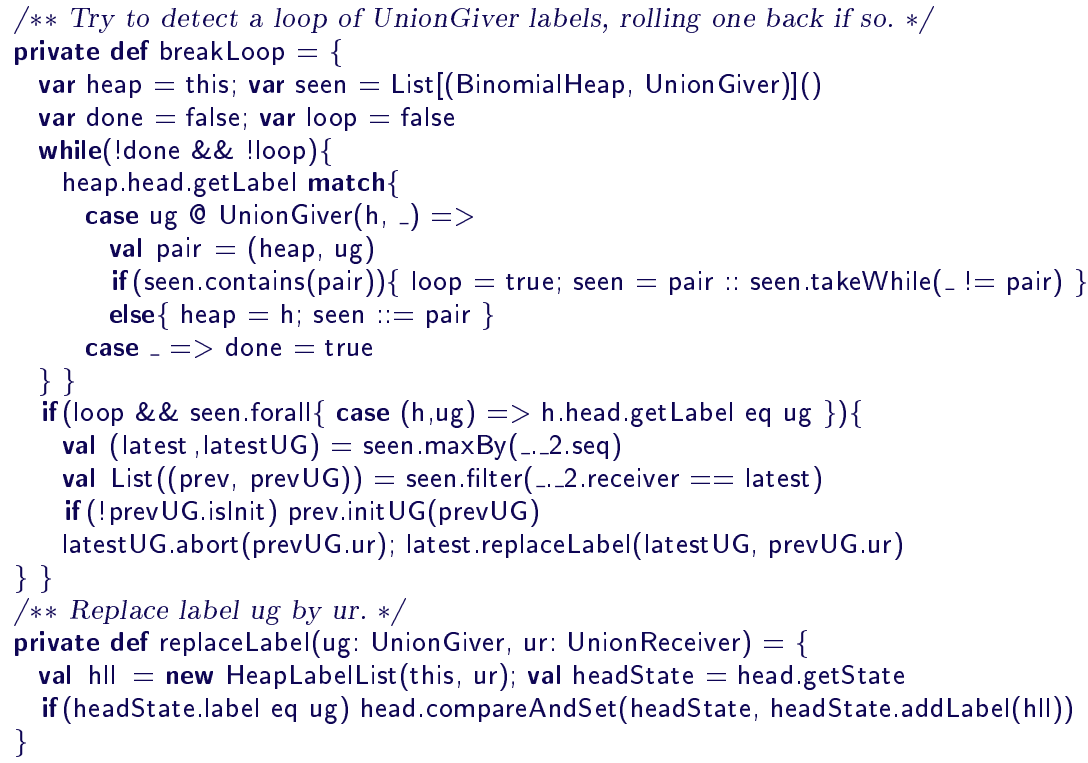

Figure 22: The breakLoop and replaceLabel functions.

path of heaps and corresponding labels (in reverse order) in the variable seen. If it returns to a pair that it has seen previously it has detected a potential loop; it extracts the sub-path corresponding to that loop. It then checks that each of the heaps is still labelled with the previously seen UnionGiver label.

The choice of which UnionGiver label in the loop to backtrack is fairly arbitrary. However, we ensure that all threads select the same label. To this end, each UnionGiver label includes a sequence number seq. All threads select the label latestUG with maximum sequence number to roll back. The (unique) pair (prev, prevUG) that is blocked by latestUG is found. If necessary, prevUG is initialised. Then latestUG is marked as aborted, to be replaced by the UnionReceiver label in prevUG (see Figure 20); this ensures that all other threads that encounter latestUG know it is being aborted, and know which label to replace it by; note that all threads that detect the loop pass in the same value for the replacement label. The replacement itself is done by replaceLabel, replacing the UnionGiver by a new HeapLabelList containing ur.

We now give correctness results for the above code; proofs are in [6].

Lemma 36. Suppose a call to breakLoop aborts a particular UnionGiver label latestUG, replacing it by a UnionReceiver label prevUG.ur. Then

1. latestUG.ur has not been added to a node;

2. prevUG has been initialised, but not aborted, at the point of replacement.

Note, in particular, that the above lemma implies that once a node is labelled with a UnionReceiver label, the corresponding UnionGiver cannot be aborted. 


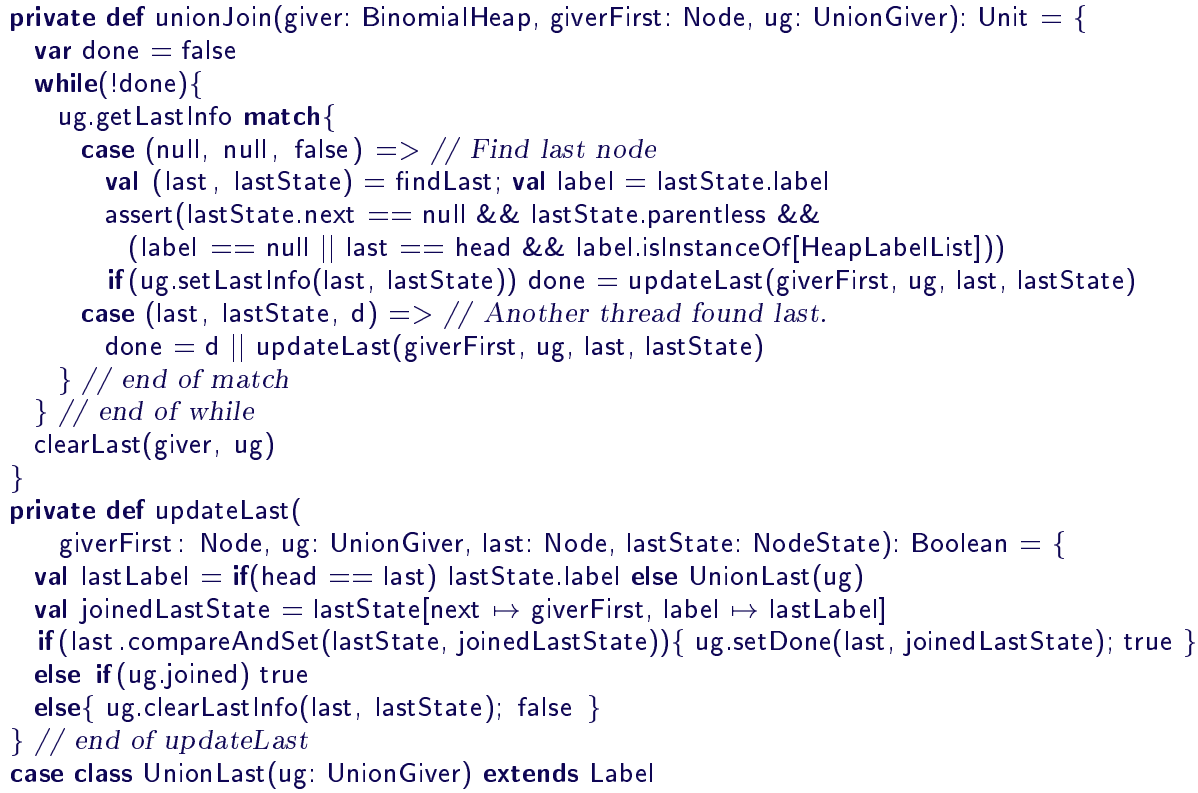

Figure 23: The unionJoin and updateLast functions, and the UnionLast class.

Lemma 37. Suppose a call to unionLabelReceiver is made following the stated precondition, and that the call adds the corresponding UnionReceiver label. Then

1. The UnionGiver label has not been aborted;

2. The UnionReceiver label is not added twice.

\subsection{Joining the heaps}

The joining CAS, and the subsequent update to the UnionGiver label, are coordinated by the function unionJoin (Figure 23). This step is made more complicated by the necessity to allow helping threads, and to ensure that two such threads do not perform successful CASes from different nodes. To this end, the UnionGiver class (Figure 24) contains a variable lastlnfo containing an atomic reference to a triple of one of three forms:

1. (null, null, false), indicating that the joining CAS has not yet happened, and the last node of the receiving heap is not currently stored;

2. (last, lastState, false), indicating that last was identified as the last node of the receiving heap, with state lastState, but that the joining CAS might not yet have happened; threads may attempt the joining CAS from lastState;

3. (last, lastState, true), indicating that the joining CAS has been done upon node last, producing state lastState.

Each thread starts by reading lastlnfo. If it receives a result of form 1, it finds the last node and its state, using findLast; we adapt this function slightly from 


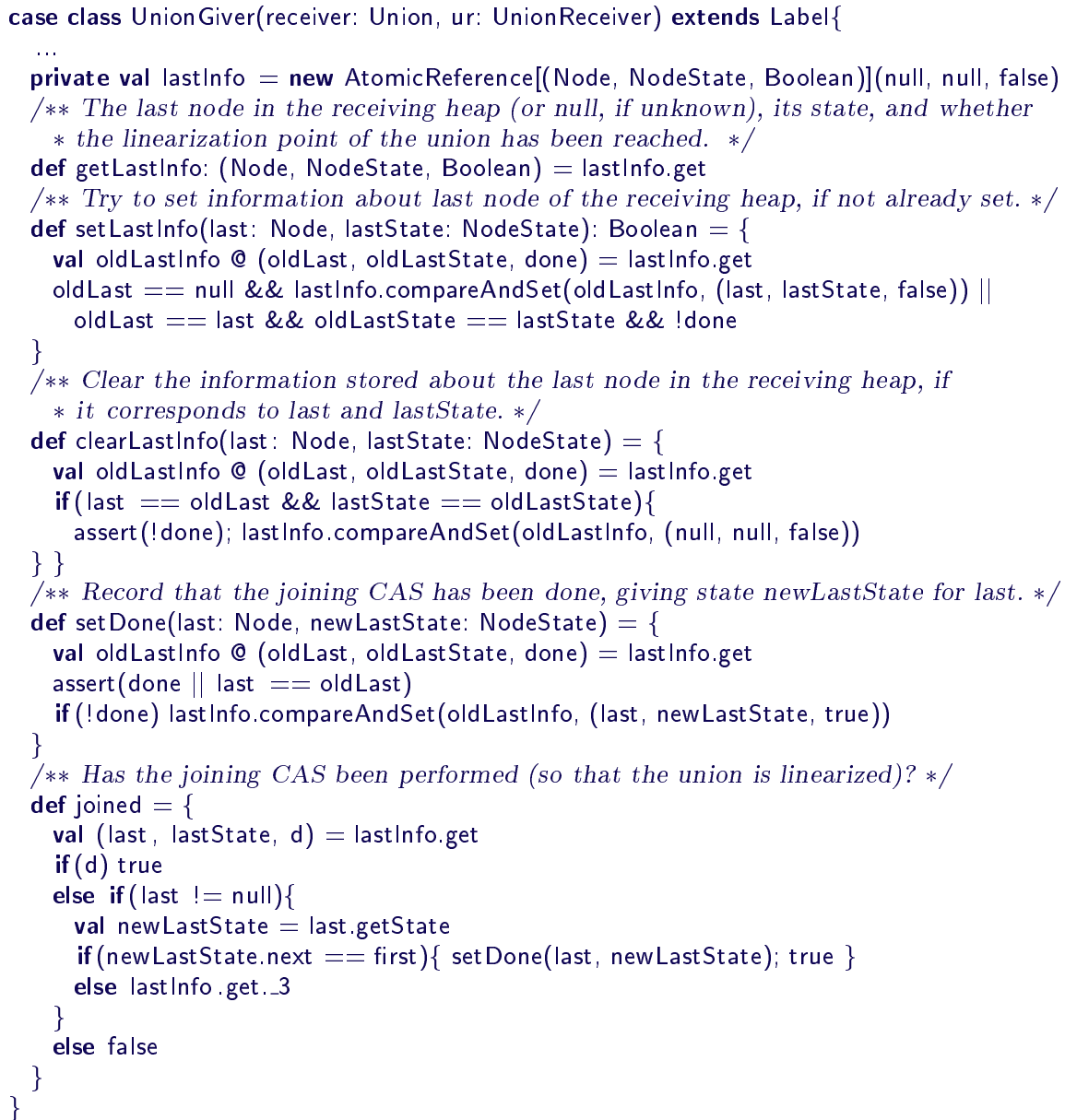

Figure 24: The UnionGiver class (continued).

the version in Section 7 to allow it to return a node labelled with a HeapLabelList, which will be the case when the receiving heap is empty. It then tries to update lastInfo to form 2; this fails (and returns false) if another thread has updated lastInfo to a different value. If the update is successful, it then tries to perform the CAS itself using updateLast (see below). Alternatively, if the read of lastlnfo returns a node and state stored by another thread, if the CAS is not recorded as having been done, it again attempts that CAS using updateLast.

The updateLast function attempts to update the last node, setting the next reference to point to the first proper node of the giver heap, and adding a UnionLast label if the receiving heap is non-empty (or else retaining the HeapLabelList). If this succeeds, it updates lastInfo via setDone, into form 3. Recall that if the CAS succeeds, it is the linearization point for the union operation. 


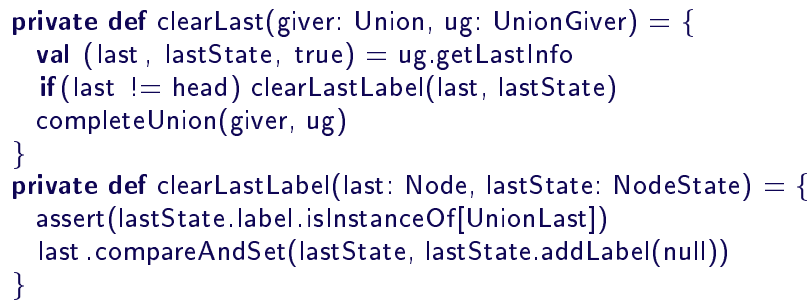

Figure 25: The clearLast and clearLastLabel functions.

If the joining CAS fails, the function tests whether another thread has performed it, using the joined function on the UnionGiver label. Otherwise, it clears lastInfo, resetting it to form 1 if no subsequent update has been performed on it.

The joined function tests whether last Info is in form 3 , indicating that setDone has been called. Otherwise, if last Info is in form 2, it tests whether the stored last now points to the first node of the giving heap; if so it calls setDone to record this fact. If last does not point to first, it re-reads lastlnfo in case, between lines 28 and 32, another thread called setDone, and subsequently the next reference of last was updated. Informally this logic is correct because last continues to point to first until after setDone is called; we justify this below.

Rule 7. The only change allowed on a node $n$ with a UnionLast(ug) label is to remove the label, which must be after a call to ug.setDone passing $n$ and its state.

The UnionLast label is removed from the former last node of the receiving heap, if applicable, using the function clearLast (Figure 25). The CAS is guaranteed to succeed unless another thread has already performed it, by Rule 7 .

Lemma 38. The joining of the heaps works correctly: at most one joining $C A S$ succeeds, joining the last node of the receiving heap to the first node of the giving heap; and calls to joined return true precisely after this CAS.

Proof: Figure 26 gives a state machine to illustrate the joining of the heaps. Each state is labelled with a 4-tuple (lastInfoForm, lastStateCorrect, joined, lastLabelled), where:

- lastInfoForm is either 1, 2 or 3, indicating the form of last Info.

- lastStateCorrect (in the cases lastInfoForm $\neq 1$ ) is a boolean indicating whether the state lastState stored in lastInfo matches the current state of last.

- joined indicates whether a successful joining CAS has occurred.

- lastLabelled, in the case that joined is true, is either $L$ indicating that last is labelled with a UnionLast label, $R$ indicating that such a label has been removed, or $E$ indicating that the receiving heap was empty.

We omit from the state diagram calls to setLastlnfo and clearLastInfo that do not change lastInfo, and unsuccessful joining CASes. Note that: 


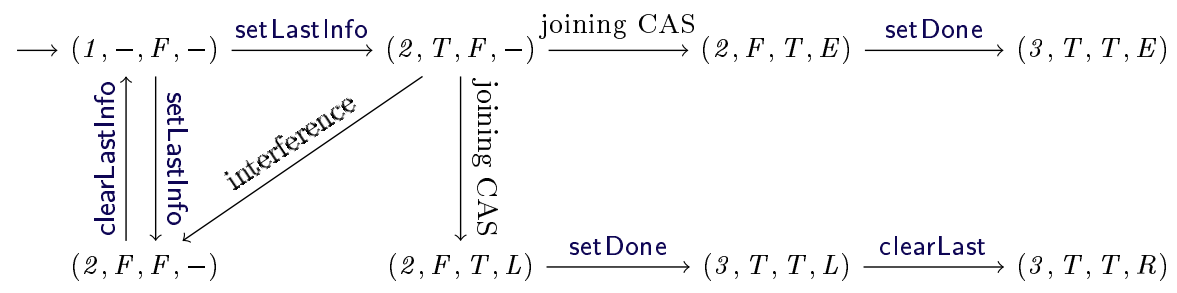

Figure 26: A state machine illustrating the joining of the heaps.

- The state diagram captures the logic of the functions on lastlnfo.

- setLastInfo may make lastStateCorrect true or false, depending on whether the state changed since the thread read it. Interference from other threads, changing the state of last, can make it false, but cannot make it true.

- clearLastlnfo is called only after an unsuccessful joining CAS, so if it changes lastInfo, there must have been interference making lastStateCorrect false.

- The joining CAS can succeed only if the value of lastState used is still valid, which implies there has been no interference.

- After the joining CAS has occurred, last.next cannot change before the UnionLast label has been removed, by Rule 7, or before setDone is called, by Rule 4 (in the case of a non-empty or empty receiving heap, respectively).

- In the states described in the previous item, a call to joined returns true, so a thread whose CAS fails does not call clearLastlnfo. Hence lastlnfo cannot change before a call to setDone; and no subsequent joining CAS can succeed.

- After the call to setDone, subsequent calls to joined again return true; and calls to getLastlnfo return a result of form 3; so again no subsequent joining CAS can succeed.

- In the case of a non-empty receiving heap, the first attempt to remove the UnionLast label succeeds, by Rule 7; and this is performed only after setDone is called.

\subsection{Completing the union}

Most of the code relating to heap indirections is in Figure 27. Each HeapIndirection object has a variable next which references either a heap, or another heap indirection ${ }^{13}$. Recall that each heap $h$ has a variable heaplndirection which references a Heaplndirection; this in turn references $h$, except temporarily during

\footnotetext{
${ }^{13}$ Values of the type Either[BinomialHeap, Heaplndirection] are either of the form Left(h) where $\mathbf{h}$ is a heap, or of the form Right(hi) where hi is a Heaplndirection.
} 


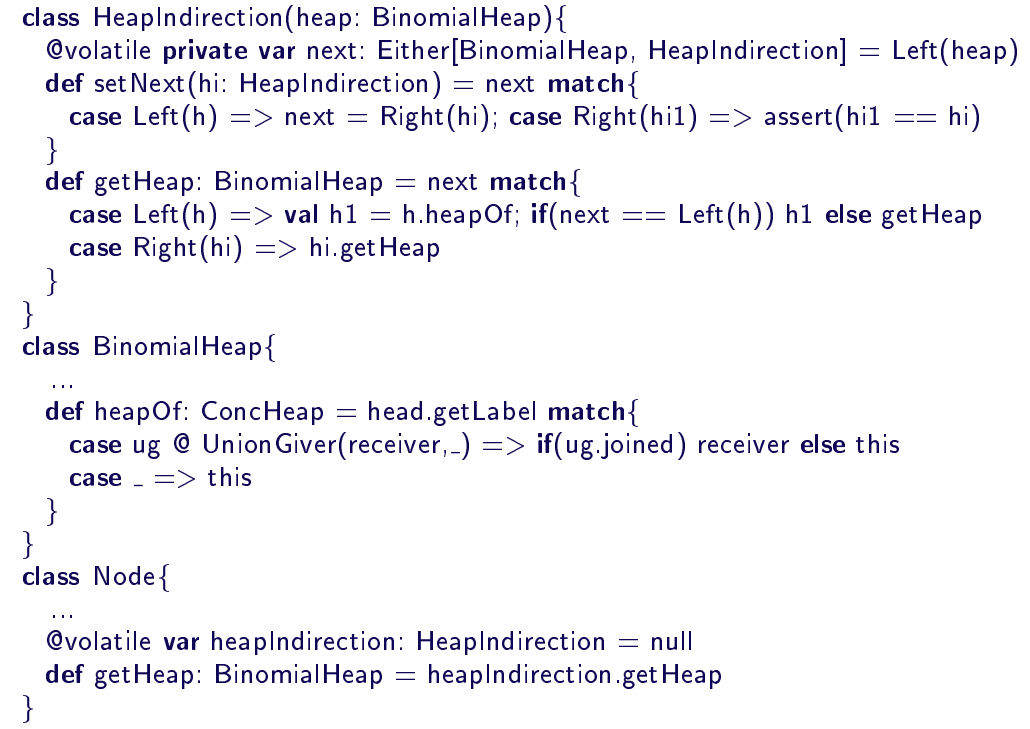

Figure 27: Code relating to Heaplndirections.

a union. Each node has a variable heaplndirection, which is set to point to the relevant heap indirection before the node is inserted; see Section 12.1.

The getHeap operation on a node calls the getHeap operation on its HeapIndirection. This follows a path of next references until it reaches the corresponding heap. The heapOf operation on the heap normally returns that heap; however, if it is currently the giving heap in a union that has been linearized, then it returns the receiving heap. On receiving the result of heapOf, getHeap checks whether its next reference has changed in the meantime, restarting if so. Thus getHeap on a node always returns the correct heap; see Lemma 40. An enhancement is to perform path compression, replacing a path of next references by a single link to the last Heaplndirection on that path; we omit the details.

The completeUnion operation (Figure 28) starts by calling completeUnionGiver, which operates on the giving heap. This records the current heap indirection of the receiver. If the heap's head is still labelled with the UnionGiver label (so another thread has not completed these steps), and if the heap indirection is the same as that recorded at the start of the union, it is updated to point to the receiver's heap indirection, and replaced by a new heap indirection.

Next, completeUnionOnGiver increments the epoch from the value stored in the UnionGiver label. It then updates the header node to remove the label and set the next reference to null.

Finally, completeUnion calls clearUR to mark the UnionReceiver label as done, and remove it from the HeapLabelList.

The following two lemmas are proven in [6]. The latter makes an assumption about nodes' heap indirections being initialised correctly, which we discharge in 


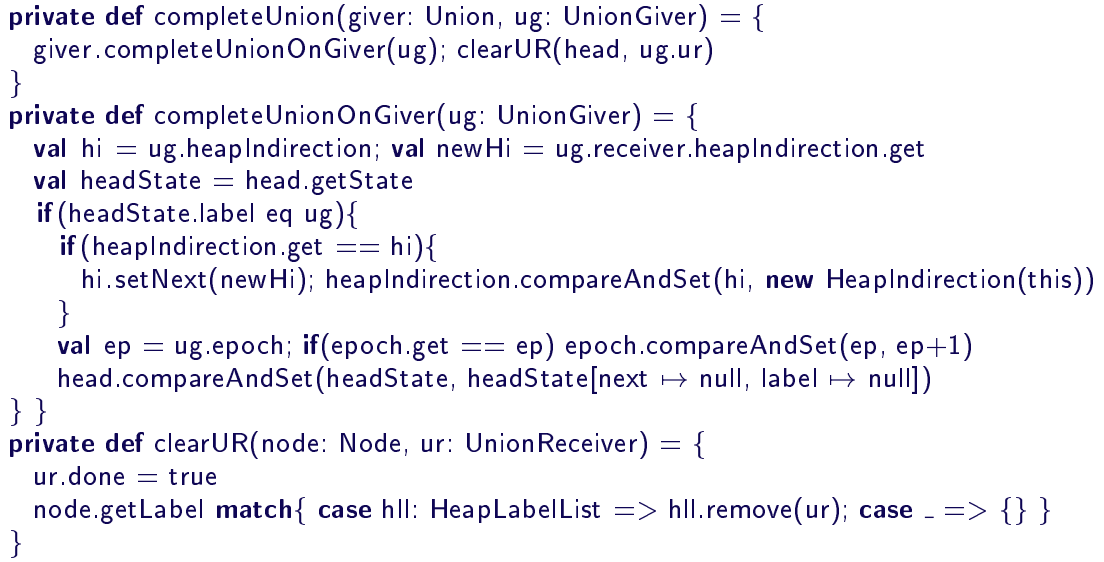

Figure 28: The completeUnion, completeUnionOnGiver and clearUR functions.

Lemmas 43 and 44 .

Lemma 39. The code in Figure 28 correctly implements steps $6-9$ of the union.

Lemma 40. Suppose that whenever a node is inserted into heap $h$, its heap indirection is set to h.heaplndirection; then the getHeap function on a node always returns the correct heap.

\subsection{Helping with a union}

Figure 29 contains the code for helping with a union. (We explain the case of Inserted labels in Section 12.1.) Helping with a UnionLast label is straightforward: it calls setDone, then clears the label.

The subsidiary function helpUG helps with a UnionGiver label ug; this simply calls the appropriate sub-function. Helping with a HeapLabelList amounts to calling the close operation on it: recall that this helps with all the operations it contains, and then removes that label. The function helpUnionReceiver is called by a HeapLabelList, either when it is being closed, or if another thread is trying to add a different UnionReceiver; this again calls the appropriate sub-function

\subsection{Correctness}

The following result follows from the previous lemmas; see [6].

Proposition 41. The union operation works correctly: the overall effect is to transfer the keys of the giving heap to the receiving heap.

We now state a liveness result. Recall from the Introduction that our approach is to prove that the operation completes under the assumption that no other thread makes any update to any node; here, we consider the addition of a label to a HeapLabelList to be an update on the corresponding node. The proof is again in [6]. 


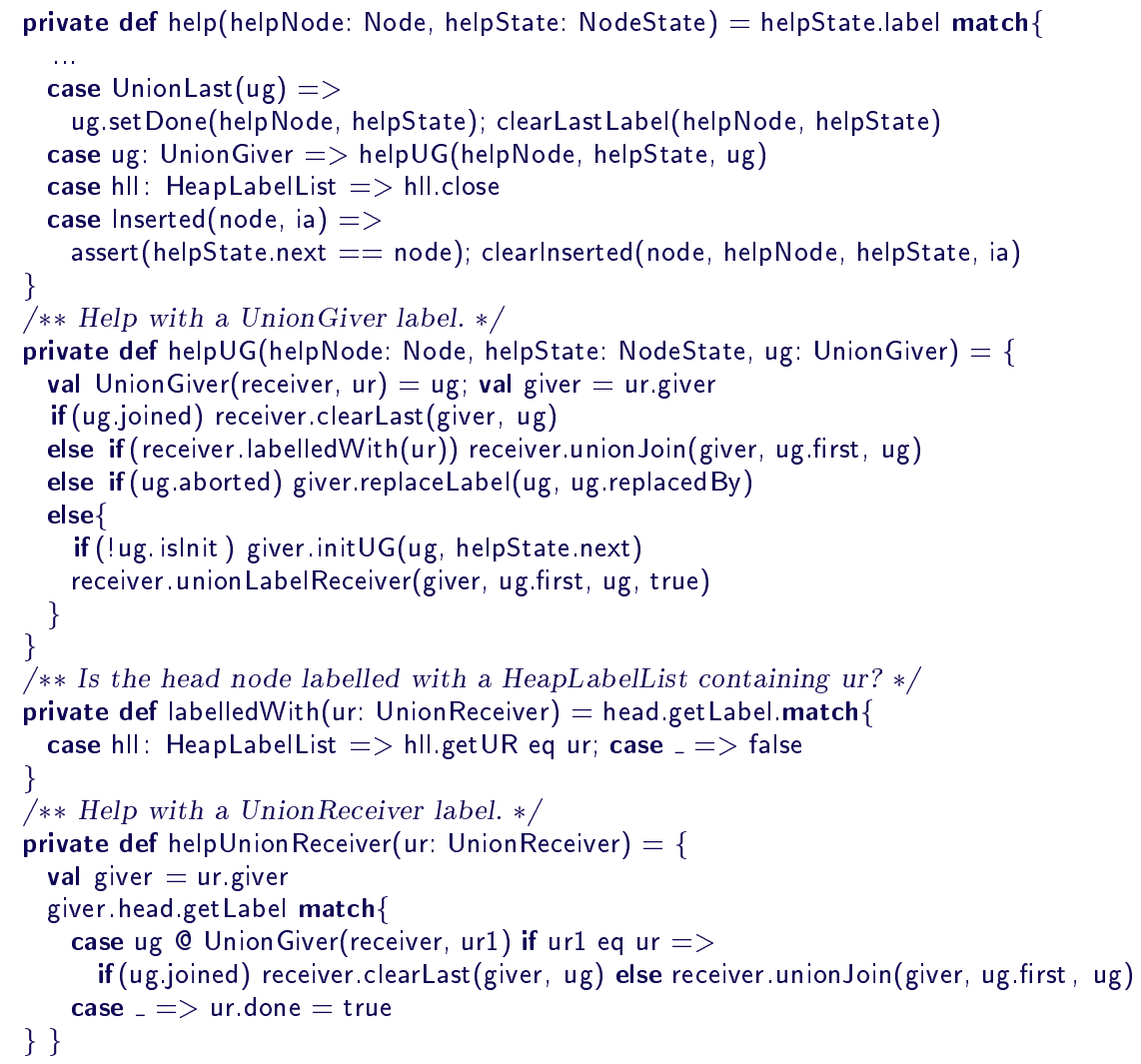

Figure 29: Helping with a union.

Lemma 42. Consider a call to union such that, from some point on, no other thread makes an update to any node. Then union completes after a finite number of steps other, perhaps, than steps involved in helping other operations. Likewise, any attempt to help with a union completes after a finite number of steps other, perhaps, than recursively helping.

\section{Adapting operations to deal with concurrent unions}

In this section we discuss how to adapt the code for other operations to deal with concurrent unions.

Each operation on the receiving heap is unaffected (other than maybe having to help with a union). The union just has the effect of adding several nodes to the end of the root list, and the implementations already deal with this.

Also, merge is unaffected. If a thread executes tidy on the giving heap in a union, it may end up merging two trees in the receiving heap: but this is completely harmless. Note that tidy never returns to the head node of its heap 
after it has started traversing: to do so would risk merging nodes that were added to the giving heap after the union with nodes in the receiving heap.

\subsection{Insertion}

Insertion is somewhat harder. If the heap is empty and the head is labelled with a HeapLabelList, we allow the new node to be inserted after the head (as is allowed by Rule 4). While traversing to find a place to insert, we ignore the other types of labels we have introduced to support unions (including the Inserted labels that we introduce below): none can appear on the last node of the heap, so there is no need to help.

Further, if while traversing the thread discovers that the current node has been transferred to another heap, because of a giving union, it restarts the traversal. However, it might be necessary to help to complete the union, to ensure lock-freedom, as follows:

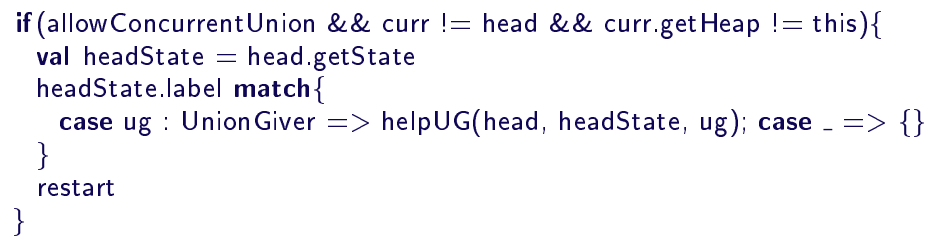

The variable allowConcurrentUnion is true if giving unions may be performed concurrently to other operations.

Recall, from Section 11, that we need to prevent the critical step (the inserting CAS) from happening concurrently to a giving union, or else the insertion may incorrectly happen on the receiving heap. To this end, we guard the critical steps by placing a suitable HeapLabel (Figure 17) on the header node within a HeapLabelList, thereby preventing a concurrent giving union. We consider, in turn, the two different ways of inserting a node.

For the case of inserting the new node below a root node of degree 0 , we replace lines 21-23 of Figure 12 by

if (insertBelow(myNode, curr, currState)) return

else myNode.state $=$ myNode.state[parent $\mapsto$ null]

where the code for insertBelow is in Figure 30. When allowConcurrentUnion is false, insertBelow acts equivalently to as in Section 6. When it is true, the code starts by recording the heap's epoch. If then checks that parent is still in the current heap. Assuming so, it sets the node's heap indirection to be that of the heap.

If then attempts to add a suitable InsertBelow label in a HeapLabelList to the heap's head node, via the function labelHead. If (in the case of insertBelow) parent's state changes, or this heap is the giving heap in a union, then the attempt to insert would fail (similar considerations apply with other functions, below). Therefore insertBelow passes parent and its state, and the current epoch to labelHead, so that if it detects a change it can fail (and return false). Otherwise, if the head has a null label, it attempts to add a new HeapLabelList containing the HeapLabel. If the head has an existing HeapLabelList, it attempts to add the HeapLabel to it. In other cases (including a UnionGiver), it helps with the label. 


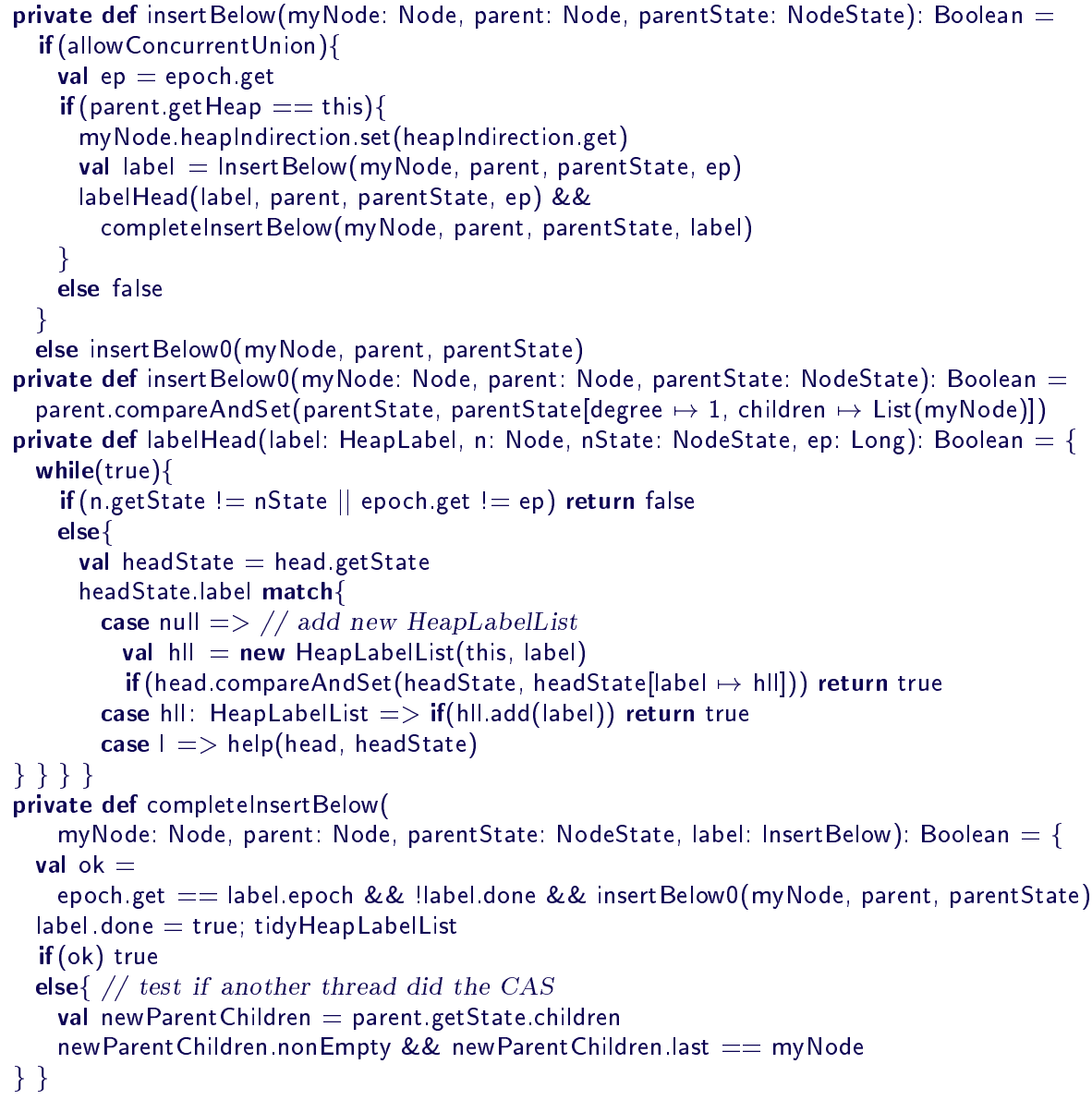

Figure 30: insertBelow and related functions.

If the labelling succeeds, insertBelow calls completelnsertBelow; other threads may help with this part. It first checks that the epoch is unchanged, which implies that parent is still in the current heap. It then checks that label has not been marked as done, as an optimisation. Finally, it tries to perform the inserting CAS. Whether or not these steps succeed, it marks label as done, and tidies the HeapLabelList. If the CAS did not succeed, it needs to test whether some other thread has performed it; this is the case if and only if myNode is now the last child of parent.

Lemma 43. Inserting as in Figure 30 works correctly. In particular: myNode is inserted into the heap this on which insert was called; myNode's heap indirection is set correctly; and the primary thread receives the correct result.

We now consider the case of inserting at the end of the root list. We replace 


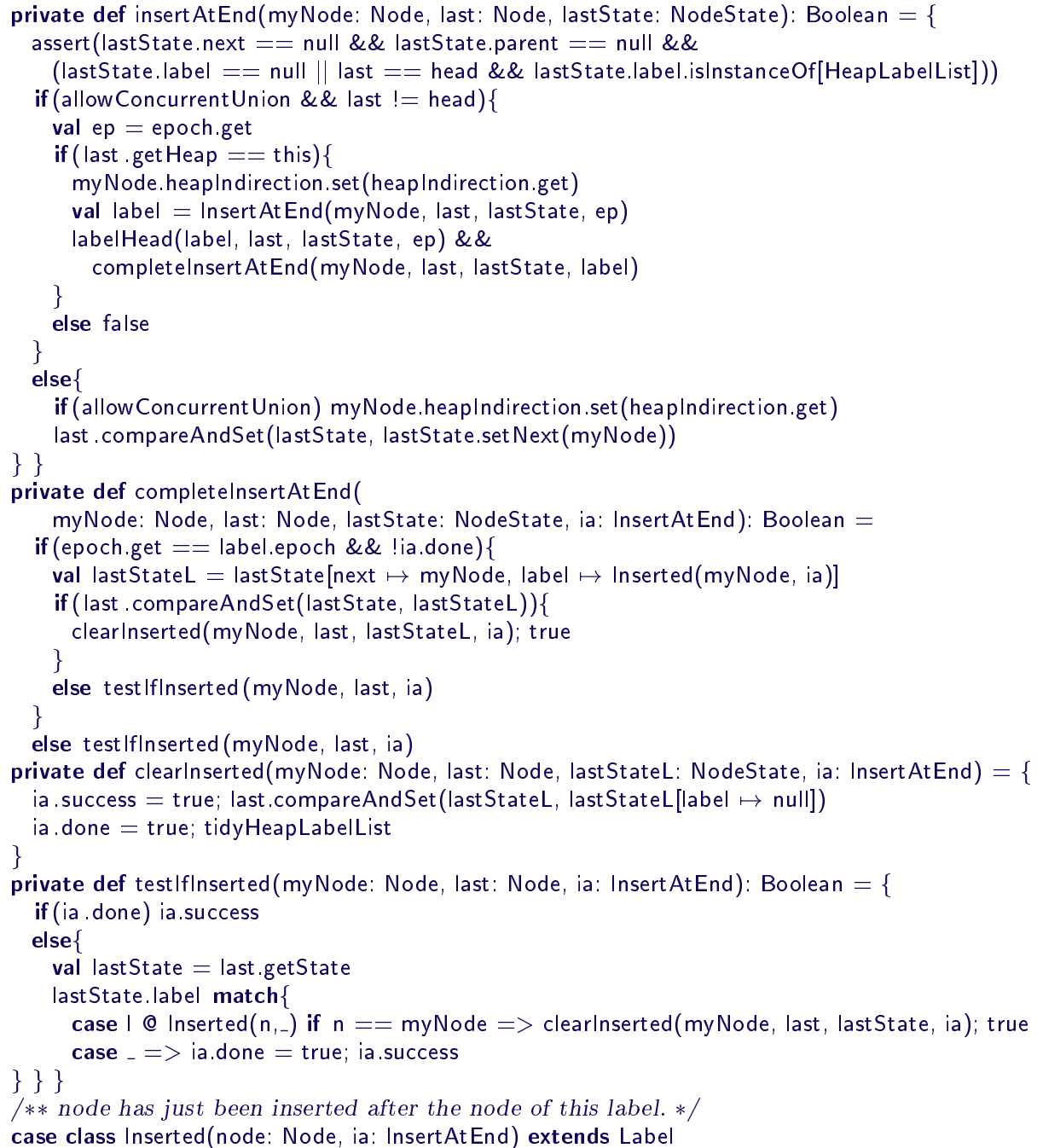

Figure 31: insertAtEnd and related functions, and the Inserted class.

lines $15-17$ of Figure 12 by

if(insertAtEnd(myNode, curr, currState)) return else BackOff()

where the code for insertAtEnd is in Figure 31. We call insertAtEnd in the same way if the heap is empty and the head is labelled with a HeapLabelList.

If allowConcurrentUnion is false, the operation is equivalent to as in Figure 12. Otherwise, if last $=$ head, there is no need to add a HeapLabel: if a concurrent giving union happens, the state of head changes so the subsequent CAS fails; however, it is necessary to set the node's heap indirection. 
In the remaining case, things are slightly more complex than with insertion below a root node, because there is no convenient way of detecting whether the insertion has been done by another thread. Our approach is to add an Inserted label (Figure 31) onto last at the same point as the inserting CAS. We then set the success flag in the InsertAtEnd HeapLabel ia, before removing the Inserted label. The relevant invariant is: the inserting CAS for myNode succeeded if and only if last has an Inserted label containing myNode or ia.success is true.

insertAtEnd first records the current epoch. It then checks that last is still in the current heap. If so, it sets myNode's heap indirection. It then calls labelHead to try to add an InsertAtEnd HeapLabel to the head, as long as last's state and the epoch do not change. If this is successful, it calls completelnsertAtEnd; other threads can help with this part.

completelnsertAtEnd checks that the epoch has not changed, and (as an optimisation) that another thread has not marked the InsertAtEnd label as done. In this case, it attempts to CAS the new node after last, adding an Inserted label at the same time. If this succeeds, it calls clearlnserted to complete the update, and returns true. If either of the previous steps fails, it calls testlfinserted, to test if another thread has completed the insertion.

clearInserted sets the success flag in the InsertAtEnd label, to indicate to other threads that the inserting CAS succeeded. It then removes the Inserted label from last. Finally, it marks the InsertAtEnd label as done. Note (Figure 29) that another thread that encounters the Inserted label can help by calling clearlnserted.

testlfInserted starts by testing if ia.done is set; if so, ia.success indicates whether the CAS was successful. Otherwise, if last has an Inserted label containing myNode, another thread did the CAS; this thread calls clearlnserted to complete the update and returns true. Otherwise, it sets the done field in ia and returns the result of ia.success.

Lemma 44. Inserting as in Figure 31 works correctly. In particular: myNode is inserted into the heap this on which insert was called; myNode's heap indirection is set correctly; and the primary thread receives the correct result.

\subsection{Minimum}

Recall that the minimum operation needs to guard against a concurrent giving union: less careful approaches risk returning a value from the receiving heap. However, if minimum detects that the joining CAS of a giving union has happened, it can immediately return None, with the operation linearized at some point between the joining CAS and the removal of the UnionGiver label, during which time the heap was empty. The revisions to the version of the minimum function in Section 8 are small, so we just explain the changes.

The revised minimum function starts by obtaining the current epoch using the function getEpoch (Figure 32). If there is a current giving union that has been linearized, then getEpoch returns None, and minimum also returns None. If getEpoch returns a value Some(startEpoch) then (as an optimisation) each time the traversal restarts, it tests whether the epoch has changed, and if so again returns None. 


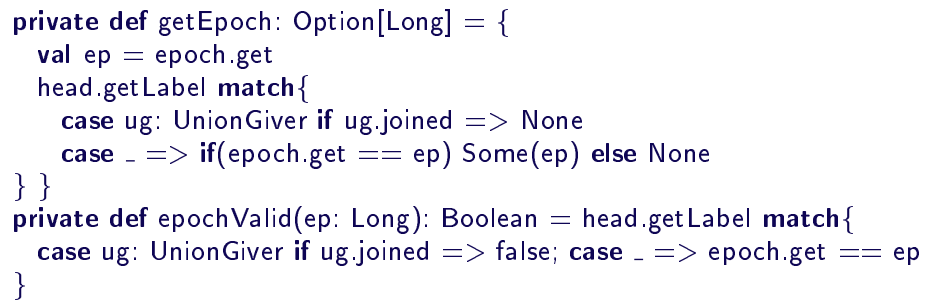

Figure 32: The getEpoch and epochValid funcitons.

The advance function (Figure 10) is adapted so that from the head node of a giving heap in a union where the joining CAS has occurred, it returns a result indicating that the heap is empty:

case ug: UnionGiver $=>$

result $=$ skipDeleted(currState.next); if(ug.joined) result $=($ null, -1 , List ()$)$

If minimum reaches the end of the traversal, and finds a candidate value to return, it again checks whether the heap has been the giving heap in a union. We replace line 20 of Figure 14 by

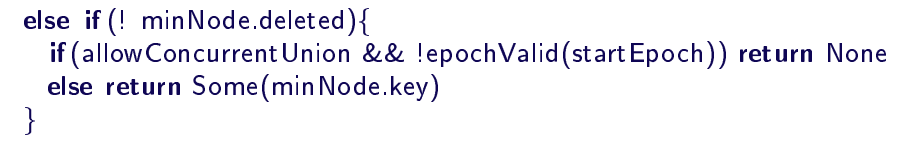

The function epochValid checks that its argument is still the valid epoch, taking into account a union that has been linearized but for which the epoch variable hasn't been incremented.

There is another subtlety. The traversal might encounter a node $n$ that was marked for deletion while it was in some other heap $h$, but $h$ has subsequently been the giving heap in a union with the current heap; in this case, it would be incorrect to return $n$ 's key. We deal with this by extending Delete labels to include a heap field, recording the heap of the deletion. We then replace line 8 of Figure 14 by the following, to ignore nodes deleted in another heap.

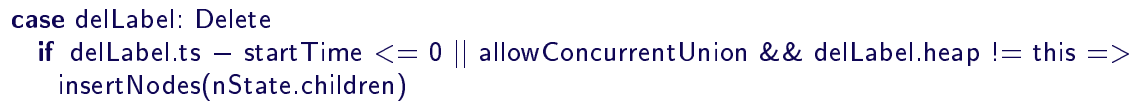

Proposition 45. The revised version of minimum works correctly, as stated in Proposition 30 and Lemma 31. Further, if minimum returns a proper result, there was no joining CAS of a giving union during the traversal.

Proof: Most of the proof is as earlier, so we just sketch the changes. The case of detecting a change of epoch and returning None was outlined above.

Suppose the function returns a proper result. Then necessarily no joining CAS of a giving union was concurrent to the traversal. The proof in Proposition 30 then goes through as before. 


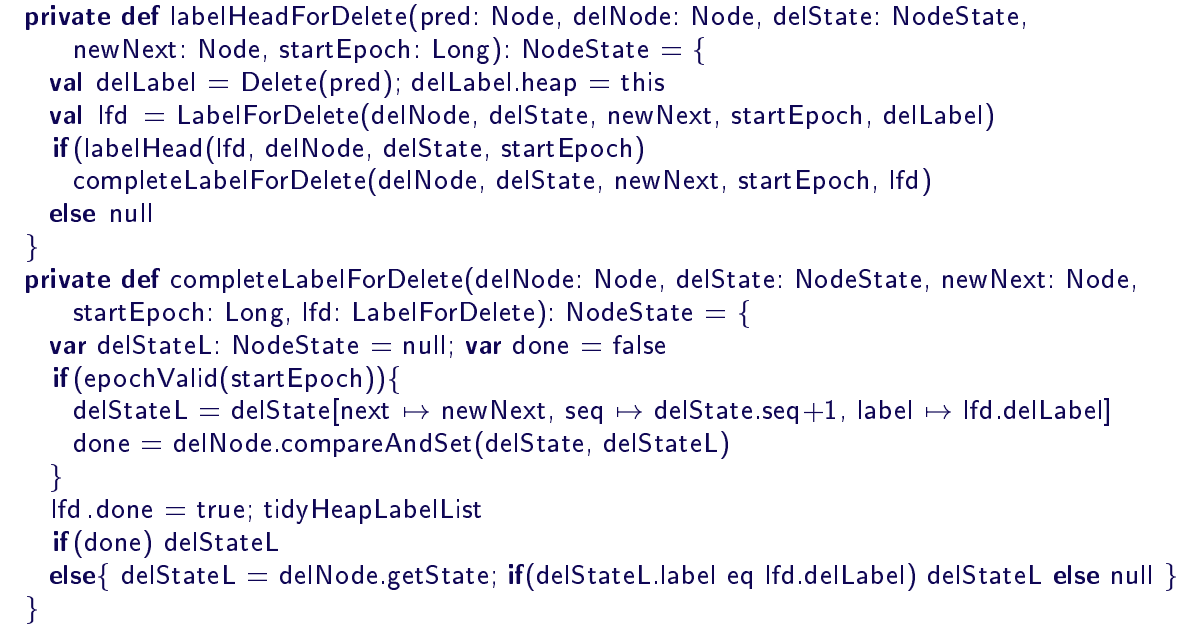

Figure 33: The functions labelHeadForDelete and completeLabelForDelete.

Finally, we explicitly avoid adding a node to minList that had been marked for deletion in a different heap. When we consider deletion (Section 12.3) we will ensure that when the marking happens, the node really is in the appropriate heap, i.e. before the joining CAS of a giving union. Further, the starting epoch is recorded before startTime; hence, if, the node were marked in the current heap and transferred to another heap before the epoch was read, then transferred back to this heap, it would have been marked before startTime and so rejected. Hence, if the value returned is marked for deletion, that marking must have happened in the current heap during the current epoch.

\section{3. deleteMin}

The traversal for deleteMin is revised in a similar way to that for minimum. At the start, the epoch is obtained using getEpoch: if this indicates that a giving union is happening, deleteMin can immediately return None. Otherwise, each time the traversal restarts, or when it finds a candidate node for deletion, if it finds that the epoch has increased, it again returns None.

Recall that we need to prevent a giving union concurrent to the labelling of the node delNode to be deleted. Our approach is similar to that for insertion: we add a HeapLabel (specifically a LabelForDelete) to the head node during the labelling of delNode. To this end, we replace line 3 of Figure 7 by

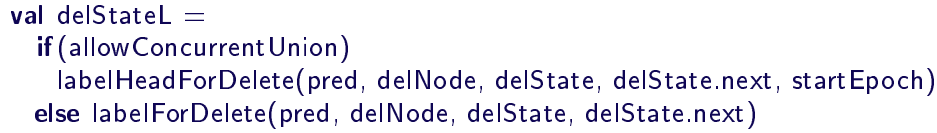

and similarly with line 16 . 


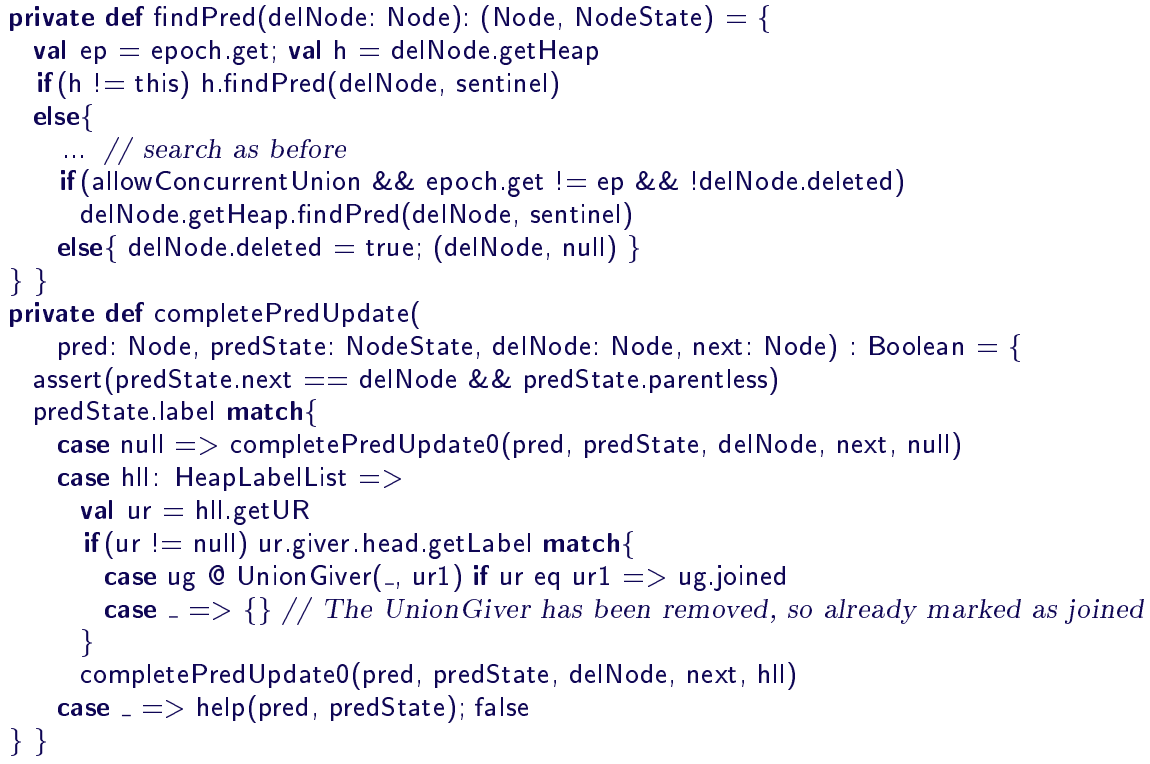

Figure 34: Revised versions of findPred and completePredUpdate; the function completePredUpdate 0 encapsulates the code in the null case of Figure 8.

The function labelHeadForDelete (Figure 33) prepares a Delete label delLabel for delNode; recall (Section 12.2) we have extended such labels with a heap field to indicate the heap of the deletion. It then creates a LabelForDelete label Ifd, and tries to add it to the head node using labelHead. If successful, it calls completeLabelForDelete; other threads may help with this.

completeLabelForDelete first checks that the epoch is not changed. If so, it tries to add the Delete label to delNode (similarly to as with labelForDelete). Whether or not, this succeeds, it marks Ifd as done. If its own CAS failed, it checks whether another thread succeeded: this is the case if and only if delNode now contains the Delete label.

Further, the function findPred (Figure 11) needs to cope with the possibility of delNode having been moved to another heap by a giving union; and if it fails to find delNode, it again needs to consider the possibility that it has been moved to another heap. To that end, findPred is revised as in Figure 34. This starts by storing, in ep, the current epoch. It then calls getHeap on delNode; if it is now in a different heap h, it recurses on that heap. Otherwise, it searches as in Figure 11. If this fails to find delNode, but the epoch has changed, and the deleted flag has not been set, it needs to search again in the new heap. Otherwise, deINode really has been decoupled, so its deleted flag can be set.

Finally, we allow completePredUpdate to be performed when the predecessor node has a HeapLabelList. However, we need to do so following Rule 4. Figure 34 does this: if a receiving union is underway, it calls joined on the corresponding 


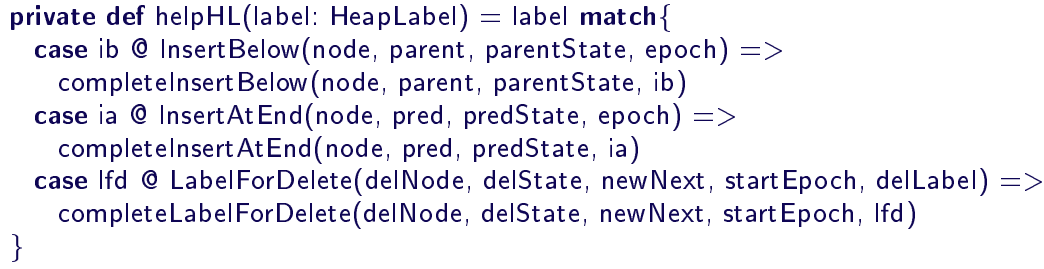

Figure 35: The helpHL function.

UnionGiver to ensure that if the joining CAS has happened, this is recorded.

Lemma 46. The revised version of findPred returns a correct result, as in Lemma 12, except without the premiss of no concurrent giving unions.

Proof: The proof is mostly as earlier. Consider the case (line 8 of Figure 34) that findPred fails to find delNode, deduces that it has been decoupled by another node and so marks it as deleted. delNode was in the current heap at line 2. Since then, the epoch hasn't changed, so step 8 of a giving union on this heap has not happened since (if the epoch was read between steps 7 and 8 of a union, then the search will be in the receiving heap). Hence, if delNode had not been decoupled, it would have been reachable from head throughout this period, and so the search would have found it.

Proposition 47. The correctness results for deleteMin (Propositions 4 and 33) still hold. Further, if deleteMin returns a proper result, there was no joining CAS of a giving union between the start of the traversal and the marking of the node for deletion.

The proof is very similar to earlier proofs; see [6].

\subsection{Helping with HeapLabels}

Figure 35 gives the function helpHL, which is called by a HeapLabelList to help to complete the operation corresponding to a HeapLabel. Each branch simply helps to complete the corresponding part of the operation.

\subsection{Liveness}

Lemma 48. Suppose, from some point on, other threads perform no updates on nodes. Then each of the new versions of operations performs a finite number of steps, other, perhaps, than helping. Likewise, each attempt to help one of these operations performs a finite number of steps other, perhaps, than recursively helping. 


\section{Linearization}

In this section we consider linearizability of the binomial heap. Note that for minimum and deleteMin operations, it is enough to consider just the final (successful) traversal. Below, we say just "traversal" to mean the final traversal. Note also that we can concentrate on a single heap: a history is linearizable if its restriction to each object is linearizable [4]; for both heaps, we linearize each union at the joining CAS.

We need to capture what are legal histories for a sequential binomial heap. We describe histories using events of the form op(args): res to represent a call to operation op with arguments args, returning res; we omit args or res when null. A sequential history is a sequence of such events. The state of a binomial heap after a history $h$ is the multiset of keys that have been added but not yet removed.

Definition 49. A sequential history $h$ is legal for a binomial heap if

- For each prefix $h^{\prime}+\left\langle\langle\right.$ minimum: None $\rangle$ or $h^{\prime}+\langle$ deleteMin: None $\rangle$ of $h$, the heap is empty after $h^{\prime}$;

- For every prefix $h^{\prime}+\left\langle\langle\right.$ minimum: Some $(k)\rangle$ or $h^{\prime}+\langle\langle$ deleteMin: Some $(k)\rangle$ of $h$, $k$ is the minimum element of the heap after $h^{\prime}$.

The following lemmas will be useful later.

Lemma 50. Suppose:

1. $h_{1}+\langle$ deleteMin: $\operatorname{Some}(k)\rangle+h_{2}+h_{3}$ is a legal sequential history;

2. $k$ is the smallest key in the heap after $h_{1}+h_{2}$;

3. $h_{2}$ contains no deleteMin: $\operatorname{Some}\left(k^{\prime}\right)$ or minimum: $\operatorname{Some}\left(k^{\prime}\right)$ operation with $k^{\prime}>k$, no deleteMin: None or minimum: None operation, and no giving union.

Then $h_{1}++h_{2}+\langle$ deleteMin: $\operatorname{Some}(k)\rangle++h_{3}$ is a legal sequential history.

Lemma 51. Consider two concurrent minimum or deleteMin operations $o p_{1}$ and $\mathrm{op}_{2}$ of keys $k_{1}$ and $k_{2}$ with $k_{1}<k_{2}$, on the same heap, such that op finishes its traversal before $\mathrm{op}_{2}$. Then $k_{1}$ must have been marked (and maybe removed from the heap) before $\mathrm{op}_{2}$ finished its traversal.

Below we talk about various events: the invocation and returns of operations; the successful CAS for an insertion; the end of the final traversal for a minimum or deleteMin; the marking of a node for deletion; the completion of the deletion; or a linearization point. As is standard, we assume that no two events occur at the same time, except we sometimes define a linearization point to be at the same time as another event of the same operation. Below we sometimes define a time $t$ to be "immediately after" some event: by this, we mean that $t$ happens after that event but before any other.

Theorem 52. The binomial heap implementation is linearizable. 
Proof: We describe, given an execution, how to build a corresponding legal sequential history suitable for linearization. Consider an intermediate state within an execution. We define the corresponding abstract state for each heap, as a multiset of keys, as follows: a key $k$ is in the multiset if it is in the heap (so the joining CAS of a giving union has not transferred it to another heap), and the instance of deleteMin that deletes the corresponding node (if any) has not yet finished its traversal. Note that this is a subset of the unmarked keys in the heap.

We start by linearizing the insert, union, deleteMin and unsuccessful minimum operations in a way that is sound with respect to this abstraction; later we explain how to add successful minimum operations, which may require moving the linearization points of deleteMin operations, and re-defining the abstract state.

- We linearize each insert or union at the point of its successful inserting or joining CAS. This corresponds to the abstract state defined above.

- We linearize each successful deleteMin at the end of its (final) traversal. Suppose it returns key $k$. Propositions 33 and 47 tell us that the node in question was a minimal unmarked node at this point; hence, since the abstract state is a subset of the unmarked keys, $k$ was the smallest key in the corresponding abstract state, as required.

- We linearize each unsuccessful minimum or deleteMin at the point, implied by Propositions 30 and 45 , or 33 and 47 , at which the heap has no unmarked keys; so again the linearization point corresponds to the abstract state.

We now seek to linearize the successful minimum operations. In most cases, we linearize the operation when the traversal finishes. However, if a concurrent deleteMin traversal has already identified the node in question as the minimum (but, necessarily, not yet completed the deletion), then we need to find a different, earlier, linearization point for the minimum; this may also require moving the linearization point of the deleteMin. We re-interpret the corresponding abstract state so that elements are removed at that new linearization point.

We pick linearization points for successful minimum operations in increasing order of keys. Suppose we have dealt with all keys $k^{\prime}<k$, and we are looking to linearise minimum operations for key $k$. Our induction hypothesis is that we have found linearization points for all insertions, unions, deleteMin operations, and unsuccessful minimum operations, and all minimum operations for keys $k^{\prime}<k$, and maybe some for key $k$ (but none with larger keys), and we have defined a corresponding abstract state, such that:

IH1. Each insert or union operation is linearized when its keys are added to the abstract state;

IH2. Each unsuccessful deleteMin or minimum operation is linearized at a point at which there was no unmarked key in the heap, and the corresponding abstract state was empty;

IH3. Each deleteMin operation giving a key $k^{\prime} \leq k$, and each minimum operation that has been linearized so far (also giving a key $k^{\prime} \leq k$ ) is linearized at a 
point at which $k^{\prime}$ was the smallest key in the abstract state;

IH4. Each deleteMin operation giving a key $k^{\prime}>k$ is linearized at the end of the traversal, at which point $k^{\prime}$ is the smallest key in the abstract state;

IH5. For each minimum operation not linearized so far (so giving a key $k^{\prime} \geq k$ ), at the end of its traversal there is no key $k^{\prime \prime}<k$ in the abstract state.

The above construction establishes the inductive hypothesis for $k=-\infty$, using Propositions 30 and 45 for IH5.

Consider a particular node $n$ with key $k$. Consider a minimum operation that returns the key from $n$. Let $t$ be the time that the corresponding deleteMin finished its traverse (including, possibly, in a different heap); or let $t=\infty$ if there is no such deleteMin.

Case 1. If such a minimum operation for node $n$ completed its traverse at time $t^{\prime}<t$, we can linearize that minimum at $t^{\prime}$. The inductive hypothesis (IH5) tells us that there was no smaller key in the abstract state at that point; and the deleting operation (if any) had not yet finished its traversal, so $k$ is still in the abstract state.

Now consider those calls to minimum (that return the key from $n$ ) that complete their traverse at some time $t^{\prime}>t$. Let $t_{\text {mark }}>t$ be the time that $n$ is marked for deletion. Note that each such minimum started before $t_{\text {mark }}$, by Lemma 31; but $t^{\prime}$ might be either before or after $t_{\text {mark }}$. Let $t_{\text {ins }}$ be the time of the linearization point of the first insertion ${ }^{14}$ after $t$ of a key $k^{\prime}<k$, or $\infty$ if there is no such insertion (if $t_{i n s}<t^{\prime}$, then that key must have been deleted before $t^{\prime}$ ).

Case 2. If the start point of a minimum operation for node $n$ is before $t_{\text {ins }}$, then we linearize it at some time $t_{L P}$ after it started and before each of $t_{\text {mark }}, t^{\prime}$ and $t_{i n s}$. We will linearize the deleteMin at a time later than $t_{L P}$, and remove $k$ from the abstract state at that time: see below. This is illustrated in the figure below (where "LP" indicates the linearization points, and $t_{i n s}, t_{\text {mark }}$ and $t^{\prime}$ may occur in any order).

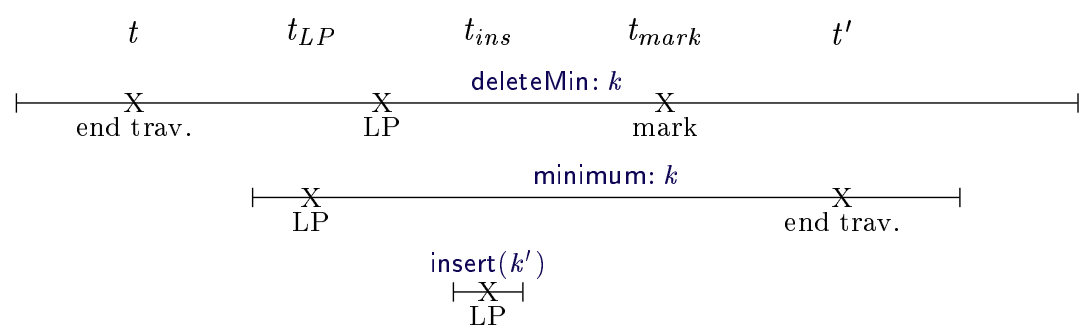

By the inductive hypothesis (IH3), $k$ was the smallest key in the abstract state at $t$ (since the deleteMin giving $k$ was previously linearised at $t$ ). No smaller

\footnotetext{
${ }^{14}$ For the rest of this proof, when we talk about insertion of keys, we intend it to include receiving union operations.
} 
key is inserted before $t_{L P}$; and $k$ is not removed before $t_{L P}$. There is no giving union between $t$ and $t_{\text {mark }}$ by Proposition 47 . Hence $k$ is still the smallest key in the (updated) abstract state at $t_{L P}$ (re-establishing IH3 for this minimum).

We now consider the linearization point for deleteMin.

a. If the start point of every such minimum is before $t_{i n s}$, then we linearize the deleteMin immediately after the last linearization point for such a minimum (as illustrated above). As above, $k$ is the smallest key in the abstract state at this point (re-establishing IH3 for this deleteMin). We need to show that moving the linearization point of the deleteMin cannot invalidate the linearization point of any other deleteMin or minimum operation. By Lemma 50, it is enough to show that the deleteMin has not been moved past any deleteMin or minimum that is unsuccessful or returns a key $k^{\prime \prime}>k$.

- The key $k$ is in the heap, unmarked, throughout this period, so (by IH2) there can be no such unsuccessful operation.

- By the induction hypothesis, there is not yet any linearization point for a minimum operation for a key $k^{\prime \prime}>k$ in the sequential history.

- By IH4, any deleteMin for key $k^{\prime \prime}>k$ is currently linearized when it finishes its traversal; and by Lemma 51, part 1, no such deleteMin operation can have completed its traversal between $t$ and $t_{\text {mark }}$.

Hence moving the linearization point of the deleteMin in this way preserves the legality of the sequential history.

We also need to prove that moving the linearization point does not falsify IH5 for any minimum operation for a key $k^{\prime \prime}>k$. However, no such minimum can complete its traversal between $t$ and the new linearization point for the deleteMin: if it had, it would have seen $k$ unmarked, and so returned $k$ rather than $k^{\prime \prime}$.

b. If some such minimum starts after $t_{\text {ins }}$, we linearize the deleteMin as in case 3 , below.

Case 3. The final case arises if a key $k^{\prime}<k$ is inserted with linearization point at some time $t_{\text {ins }}$ after $t$, and a minimum (on node $n$ ) starts after $t_{\text {ins }}$; necessarily $t_{\text {ins }}<t_{\text {mark }}$ since the minimum starts before $t_{\text {mark }}$ (Lemma 31 ). See figure below (again $t^{\prime}$ might be either before or after $t_{\text {mark }}$ ).

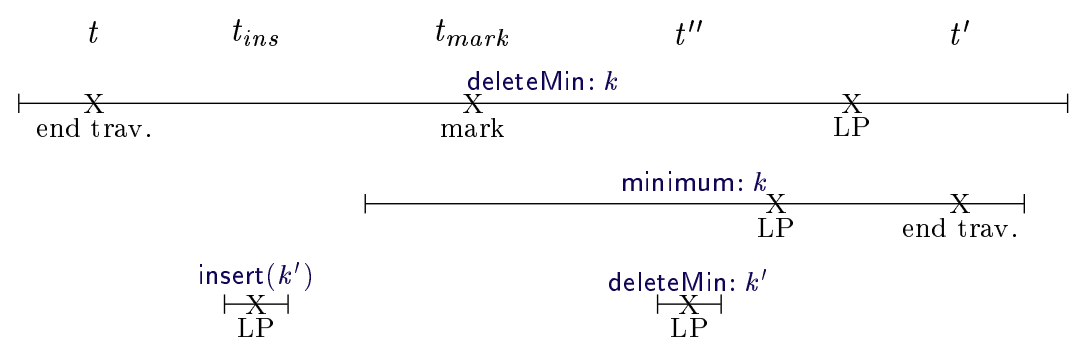

No giving union can occur between $t$ and $t_{\text {mark }}$ by Proposition 47, nor between the start of the traverse in minimum and $t^{\prime}$ by Proposition 45 ; combining these means no giving union can occur between $t$ and $\max \left(t^{\prime}, t_{\text {mark }}\right)$. 
Note that, by IH $5, k^{\prime}$ must have been removed from the abstract state before $t^{\prime}$, as must every other key less than $k$ that was inserted since $t$. Let $t^{\prime \prime}<t^{\prime}$ be the time of the last linearization point for such a deletion $\left(t^{\prime \prime}\right.$ might be either before or after $\left.t_{\text {mark }}\right)$. So, immediately after $t^{\prime \prime}$, the abstract state holds no key less than $k$ (by IH4). We linearize each such minimum immediately after $t^{\prime \prime}$ (so before $t^{\prime}$ ) in some order; and linearize the deleteMin immediately after the last such; this re-establishes IH3 for both operations.

The linearization point for the deleteMin is within its operation: the deleteMin is linearized before the last $t^{\prime}$, which is before the node $n$ is fully deleted, which is before the end of the deleteMin operation, as required.

We need to show that moving the linearization point of the deleteMin does not invalidate the linearization point of any other operation. As in the previous case, it is enough to show that the deleteMin has not moved past any other deleteMin operation that is unsuccessful or returns a key $k^{\prime \prime}>k$, or past an unsuccessful minimum operation. By IH2 and IH4, each such operation is linearized at the end of its traversal. But no such operation can finish its traversal between $t$ and $t^{\prime \prime}$ : between $t$ and $t_{m a r k}$, that traversal would have seen $k$ unmarked, and so returned $k$ instead of failing or returning $k^{\prime \prime}$; and between $t_{\text {ins }}$ and $t^{\prime \prime}$, there was a smaller unmarked key (e.g. $\left.k^{\prime}\right)$ in the heap. Hence shifting the linearization point preserves the legality of the sequential history.

We also need to show that moving the linearization point does not falsify IH5 for any minimum operation for a key $k^{\prime \prime}>k$. However, no such minimum can complete its traverse between $t$ and the new linearization point for the deleteMin of $k$ : as in the previous paragraph, it would have seen a smaller unmarked key, and so returned that instead of $k^{\prime \prime}$.

\section{Lock-freedom}

In this section we prove lock-freedom. We need to consider chains of helping, where a thread helping with one operation is forced to help another operation.

Lemma 53. Suppose, from some point on, other threads make no update to nodes. Then each chain of helping is finite (so acyclic).

Proof: The only types of labels for which helping may lead to recursively helping with another label are Delete, UnionGiver and UnionReceiver (within a HeapLabelList).

We show that any chain of helping traverses a finite sequence of heaps. Helping a UnionGiver label can require helping the label on the head of the receiving heap; if the latter is also a UnionGiver label, this can lead to recursive helping of its receiving heap; however, the code in breakLoop is designed to detect and break what might otherwise be a loop of unions helping one another. In addition, helping a UnionGiver label can lead to helping the label on the last node of the receiving heap; but the chain does not progress to a third heap from there. 
Now consider a chain of Deletes helping one another. Combining Lemmas 5 and 13, each such instance of helping is via one of the following possibilities:

- from predUpdate, a call to findPred, leading to a call to helpDelete on the parent of delNode's predecessor;

- from deleteWithParent, a call to helpDelete on delNode's parent;

- from completePredUpdate, a call to help on delNode's predecessor.

In each case the node being helped is either closer to the root list or earlier in the root list than delNode, or has already been decoupled (in which case it's the final node in the chain of helping); thus this chain of Deletes must be finite.

If the chain reaches a node with a UnionGiver label, necessarily via the third case above, threads help with that. However, as above, helping never leads back to a node in this heap, so this does not lead to a cycle of helping.

If the chain reaches a HeapLabelList then the first pred update is done: it is important that the deletes do not help with a UnionReceiver in this case, for this could lead to a loop of helping (specifically if the union is attempting the joining CAS, and all the nodes of the receiving heap have a Delete label).

Theorem 54. The concurrent binomial heap is lock-free.

Proof: Suppose, for a contradiction, that there is an infinite execution of the binomial heap, during which only a finite number of public operations (i.e. insert, minimum, deleteMin or union) complete. This assumption means that only a finite number of new calls are made to tidy (since tidy is called only when an insert, deleteMin or union finishes).

We show that, from some time onwards, no updates are made to any nodes.

1. Each public operation makes a bounded number of updates to nodes $e x-$ cluding (for the moment) the addition of HeapLabels within labelHead: for example, a union makes at most five such updates. Hence, from some time $t_{0}$ onwards, no such update is made to any node.

2. Consider merge operations. From time $t_{0}$ on, only a finite number of $\mathrm{b}$ updates of merges can take place (bounded by the number of roots, minus one). Hence, from some point on, no changes are made to the root list. Thus, by Lemma 35, from some time onwards, no thread performs any step within tidy (or its subfunction merge).

3. Finally, each operation considered in step 1 needs to add a HeapLabel more than once only if some other update has interfered with the first attempt; so from some time $t$ onwards each operation adds at most a single HeapLabel. Hence from some time, no more such additions are made, i.e. no updates are made to any nodes by these operations.

Then, by Lemmas $24,26,32,34,42$ and 48 , each call to insert, union, minimum and deleteMin returns after a finite number of steps, outside of helping; and each instance of helping takes a finite number of steps, outside recursive helping. Lemma 53 shows that each chain of helping is finite. Finally, each attempt at helping makes progress, so each operation helps with each node a finite number 


\begin{tabular}{|c|c|c|c|c|c|c|c|c|}
\hline \multicolumn{3}{|c|}{$\begin{array}{l}\text { Operation probabilities } \\
\text { insert deleteMin minimum }\end{array}$} & \multicolumn{2}{|c|}{$\begin{array}{l}\text { Initial } \\
\text { size }\end{array}$} & This paper & \multicolumn{2}{|c|}{$\begin{array}{l}\text { Sundell \& } \\
\text { Tsigas }\end{array}$} & $\begin{array}{l}\text { Lindén \& } \\
\text { Jonsson }\end{array}$ \\
\hline 0.5 & 0.5 & 0.0 & \multicolumn{2}{|c|}{$10 \mathrm{~K}$} & $4012 \mathrm{~K} \pm 47 \mathrm{~K}$ & \multicolumn{2}{|c|}{$2904 \mathrm{~K} \pm 40 \mathrm{~K}$} & $1386 \mathrm{~K} \pm 137 \mathrm{~K}$ \\
\hline 0.5 & 0.5 & 0.0 & \multicolumn{2}{|c|}{$100 \mathrm{~K}$} & $3270 \mathrm{~K} \pm 29 \mathrm{~K}$ & \multicolumn{2}{|c|}{$2912 \mathrm{~K} \pm 37 \mathrm{~K}$} & $1797 \mathrm{~K} \pm 78 \mathrm{~K}$ \\
\hline 0.5 & 0.5 & 0.0 & \multicolumn{2}{|c|}{$1000 \mathrm{~K}$} & $1574 \mathrm{~K} \pm 42 \mathrm{~K}$ & \multicolumn{2}{|c|}{$2499 \mathrm{~K} \pm 42 \mathrm{~K}$} & $1854 \mathrm{~K} \pm 195 \mathrm{~K}$ \\
\hline 0.4 & 0.4 & 0.2 & \multicolumn{2}{|c|}{$100 \mathrm{~K}$} & $3531 \mathrm{~K} \pm 46 \mathrm{~K}$ & \multicolumn{2}{|c|}{$3581 \mathrm{~K} \pm 33 \mathrm{~K}$} & $2859 \mathrm{~K} \pm 234 \mathrm{~K}$ \\
\hline 0.3 & 0.3 & 0.4 & \multicolumn{2}{|c|}{$100 \mathrm{~K}$} & $3827 \mathrm{~K} \pm 43 \mathrm{~K}$ & \multicolumn{2}{|c|}{$4475 \mathrm{~K} \pm 232 \mathrm{~K}$} & $4233 \mathrm{~K} \pm 624 \mathrm{~K}$ \\
\hline 0.6 & 0.4 & 0.0 & \multicolumn{2}{|l|}{0} & $3525 \mathrm{~K} \pm 67 \mathrm{~K}$ & \multicolumn{2}{|c|}{$2144 \mathrm{~K} \pm 277 \mathrm{~K}$} & $1953 \mathrm{~K} \pm 70 \mathrm{~K}$ \\
\hline \multirow[t]{5}{*}{0.7} & 0.3 & 0.0 & 0 & & $2289 \mathrm{~K} \pm 97 \mathrm{~K}$ & $1569 \mathrm{~K}$ & $140 \mathrm{~K}$ & $1690 \mathrm{~K} \pm 67 \mathrm{~K}$ \\
\hline & \multicolumn{4}{|c|}{$\begin{array}{c}\text { Operation probabilities } \\
\text { insert deleteMin minimum union }\end{array}$} & $\begin{array}{l}\text { Initial } \\
\text { size }\end{array}$ & $\begin{array}{l}\text { Number } \\
\text { of heaps }\end{array}$ & \multicolumn{2}{|c|}{ This paper } \\
\hline & 0.38 & 0.38 & 0.19 & 0.05 & $100 \mathrm{~K}$ & 4 & \multicolumn{2}{|c|}{$1352 \mathrm{~K} \pm 10 \mathrm{~K}$} \\
\hline & 0.38 & 0.38 & 0.19 & 0.05 & $100 \mathrm{~K}$ & 16 & \multicolumn{2}{|c|}{$2773 \mathrm{~K} \pm 531 \mathrm{~K}$} \\
\hline & 0.4 & 0.4 & 0.19 & 0.01 & $100 \mathrm{~K}$ & 16 & \multicolumn{2}{|c|}{$3868 \mathrm{~K} \pm 39 \mathrm{~K}$} \\
\hline
\end{tabular}

Figure 36: Experimental results, giving throughput (in operations per second).

of times; and, by assumption, no new nodes are added, so each thread helps a finite number of nodes. Hence each operation performs a finite number of steps in total.

\section{Conclusions}

In this paper we have presented a lock-free linearizable concurrent binomial heap. Our main interest was in the development and correctness of the implementation, rather than out-and-out performance. Nevertheless, the heap gives good performance in several situations. Figure 36 gives experimental results. The experiments were run on a 32-core server (two $2.1 \mathrm{GHz} \operatorname{Intel}(\mathrm{R}) \mathrm{Xeon}(\mathrm{R})$ E5-2683 CPUs with hyperthreading enabled, with 256GB of RAM). In each execution, 64 threads performed two million randomly chosen operations each. Each row of each table corresponds to a particular choice of parameters, namely the probabilities of different operations, and the initial number of keys in the priority queue. In each case, ten executions were made, and the throughput calculated; the tables give the means and $95 \%$ confidence intervals.

The first table compares against the skiplist-based priority queues of Sundell and Tsigas [11], and Lindén and Jonsson [12]. Our implementation gives the best throughput in most cases. It performs less well when it contains a large number of keys: the longer root list has an adverse effect on performance; the corresponding effect on the skiplist-based implementations is smaller. The other implementations are more efficient on the minimum operation, since this simply selects the first key in the skiplist.

The second table includes the union operation (which is not provided by the other implementations). Throughput is still decent with reasonably infrequent union operations, and with a reasonable number of heaps. However, unsurprisingly, throughput is reduced with a small number of heaps or with frequent union 
operations: the union operation blocks the critical steps of other operations; and the HeapLabelList on the head node becomes a bottleneck.

We review some of the main challenges, and our solutions, in the hope that the solutions can prove useful in other applications. One of the biggest challenges was the problem of reliably traversing the heap, for example to find the smallest node, while other threads are rearranging it: our use of sequence numbers effectively allows one thread to signal to another that its traversal might have been corrupted. In addition, avoiding race conditions proved challenging: our use of labels to lock nodes helped avoid most of these.

Allowing giving unions concurrently to other operations presented a particular challenge. Our labelling of head nodes prevented such unions concurrently to the critical steps of those operations (while maintaining lock-freedom); but in addition, it was necessary to develop techniques to test whether a union had happened during some period, and to find the current heap containing a node; and allowing threads to help with the operation introduced additional difficulties.

There were several subtleties concerning linearizability; in particular, the fact that minimum can return a key that is marked for deletion before the traversal ends complicated the proof of linearizability. As noted in the Introduction, the techniques from [5] proved very useful in detecting subtle linearizability bugs in early versions, and strengthens our confidence in the final version.

Ensuring lock-freedom also proved challenging. In earlier versions of our implementation, we ran into a number of situations where a thread could get into a loop: for example, an attempted traversal could follow a loop of nodes because of an incomplete merge; or a chain of helping of operations that forms a loop. This difficulty is reflected in the complexity of the rank used to prove insert lock-free (Lemma 24).

We also encountered various challenges in trying to make the operations efficient. Allowing a thread to insert below an existing singleton node, rather than just at the end of the root list, removed a bottleneck. Preventing merges from using those nodes reduced interference. Our use of HeapLabelLists allowed the critical steps of operations to be concurrent to one another and to a receiving union, but not to giving unions. Nevertheless, when most insertions are of a key smaller than nearly all the keys in the heap, it becomes harder to insert below an existing node, thereby making the final root a bottleneck again; we conjecture this could be improved by using a combining funnel [18] to allow several insertions to combine together into a single update on the heap.

For deleteMin, maintaining multiple minima while traversing made a huge difference: different deleteMin calls compete for the same nodes, so many fail; conversely, compiling a list of fall-back options does not cost much. Also, allowing a thread to mark a non-root node for deletion, when its parent is being deleted, gives benefits: it means that only the thread that succeeded in the marking has to help with the deletion of the parent.

Within delete, we previously labelled both the node to be deleted and its predecessor. However, this meant that two nodes were locked, rather than one; and it also meant that there was the possibility of the deletion being disrupted 
if the nodes were separated before the labelling, or the state of the second node changed after the first node was labelled. Locking just a single node seems to work better, even though it means that sometimes an additional traversal is necessary to find the new predecessor. This change also made achieving, and proving, lock-freedom easier: previously it was difficult to avoid cycles of operations helping one another. However, we can see no easy way to similarly reduce the amount of labelling in the merge operation.

Laziness can help. When finding the predecessor of the node being deleted, we use an inexpensive but unreliable search, before falling back on a reliable but expensive one. Likewise, within insert, we avoid helping an operation on the final root on the first few traversals, in the hope it will complete anyway. We suspect similar tactics can be used elsewhere.

Our implementation used various parameters, that can be tuned. It would be useful to make these adaptive, where they vary automatically. For example, the number of minimal nodes that a thread records while traversing in minimum or deleteMin could vary based on how many such nodes were actually used in previous operations; and the frequency of tidying could vary based on the length of the root list that a thread encounters while traversing.

\section{References}

[1] J. Vuillemin, A data structure for manipulating priority queues, Communications of the ACM 21 (4) (1978) 309-315.

[2] T. H. Cormen, C. E. Leiserson, R. L. Rivest, Introduction to Algorithms, MIT Press, 1999.

[3] G. Barnes, A method for implementing lock-free shared data structures, in: Proceedings of the 5th ACM Symposium on Parallel Algorithms and Architectures, 1993, pp. 261-270.

[4] M. Herlihy, J. Wing, Linearizability: a correctness condition for concurrent objects, ACM Transactions on Programming Languages and Systems 12 (3) (1990) 463-492.

[5] G. Lowe, Testing for linearizability, Concurrency and Computation: Practice and Experience 29 (4).

[6] G. Lowe, Lock-free concurrent binomial heaps, Tech. rep., University of Oxford, http://www.cs.ox.ac.uk/people/gavin.lowe/BinomialHeap/ index.html (2017).

[7] M. Herlihy, N. Shavit, The Art of Multiprocessor Programming, Morgan Kaufmann, 2012.

[8] V. A. Crupi, S. K. Das, M. C. Pinotti, Parallel and distributed meldable priority queues based on binomial heaps, in: International Conference on Parallel Processing, 1996. 
[9] Q. Huang, W. E. Weihl, An evaluation of concurrent priority queue algorithms, in: Proceedings of the Third IEEE Symposium on Parallel and Distributed Processing, 1991, pp. 518-525.

[10] I. Lotan, N. Shavit, Skiplist-based concurrent priority queues, in: Proceedings of the First International Parallel and Distributed Processing Symposium, 2000.

[11] H. Sundell, P. Tsigas, Fast and lock-free concurrent priority queues for multi-thread systems, Journal of Parallel and Distributed Computing 65 (5) (2005) 609-627.

[12] J. Lindén, B. Jonsson, A skiplist-based concurrent priority queue with minimal memory contention, in: OPODIS, no. 8304 in LNCS, 2013, pp. 206220.

[13] Y. Liu, M. Spear, Mounds: Array-based concurrent priority queues, in: Proceedings of 41st International Conference on Parallel Processing, 2012.

[14] A. Braginsky, N. Cohen, E. Petrank, CBPQ: High performance lock-free priority queue, in: Proceedings of Euro-Par 2016: Parallel Processing, 2016.

[15] D. Alistarh, J. Kopinsky, J. Li, N. Shavit, The SprayList: A scalable relaxed priority queue, in: Proceedings of Principles and Practice of Parallel Programming (PPoPP '15), 2015.

[16] B. A. Galler, M. J. Fischer, An improved equivalence algorithm, Communications of the ACM 7 (1964) 301-330.

[17] M. Michael, M. Scott, Simple, fast, and practical non-blocking and blocking concurrent queue algorithms, in: Proc. of the Fifteenth Annual ACM Symposium on Principles of Distributed Computing, 1996, pp. 267-275.

[18] N. Shavit, A. Zemach, Combining funnels: A dynamic approach to software combining, Journal of Parallel and Distributed Computing 60 (11) (1998) $1355-1387$. 